\title{
Future Restrictions on Diesel Fuel Combustion in California: Energy and Emissions Implications of Four Scenarios
}

ARGONNE NATIONAL LABORATORY

Center for Transportation Research

Argonne National Laboratory

Operated by The University of Chicago, under Contract W-31-109-Eng-38, for the

United States Department of Energy 


\section{Argonne National Laboratory}

Argonne National Laboratory, with facilities in the states of Illinois and Idaho, is owned by the United States Government, and operated by the University of Chicago under the provisions of a contract with the Department of Energy.

This technical memo is a product of Argonne's Energy Systems (ES) Division. For information on the division's scientific and engineering activities, contact:

Director, Energy Systems Division

Argonne National Laboratory

Argonne, Illinois 60439-4815

Telephone (630) 252-3724

Presented in this technical memo are preliminary results of ongoing work or work that is more limited in scope and depth than that described in formal reports issued by the ES Division.

Publishing support services were provided by Argonne's Information and Publishing Division (for more information, see IPD's home page: http://www.ipd.anl.gov/).

\section{Disclaimer}

This report was prepared as an account of work sponsored by an agency of the United States Government. Neither the United States Government nor any agency thereof, nor The University of Chicago, nor any of their employees or officers, makes any warranty, express or implied, or assumes any legal liability or responsibility for the accuracy, completeness, or usefulness of any information, apparatus, product, or process disclosed, or represents that its use would not infringe privately owned rights. Reference herein to any specific commercial product, process, or service by trade name, trademark, manufacturer, or otherwise does not necessarily constitute or imply its endorsement, recommendation, or favoring by the United States Government or any agency thereof. The views and opinions of document authors expressed herein do not necessarily state or reflect those of the United States Government or any agency thereof, Argonne National Laboratory, or The University of Chicago. 
Future Restrictions on Diesel Fuel Combustion in California: Energy and Emissions Implications of Four Scenarios

by C.L. Saricks, D.M. Rote, F. Stodolsky, and J.J. Eberhardt*

Center for Transportation Research, Energy Systems Division,

Argonne National Laboratory, 9700 South Cass Avenue, Argonne, Illinois 60439

September 1999

Work sponsored by U.S. Department of Energy, Assistant Secretary for Energy Efficiency and Renewable Energy, Office of Transportation Technologies, Office of Heavy Vehicle Technologies 
This report is printed on recycled paper. 


\section{Contents}

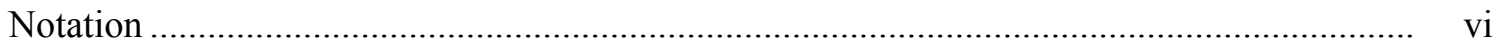

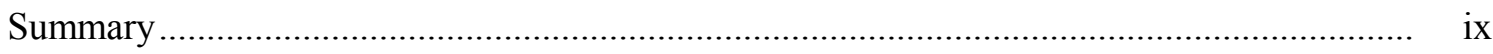

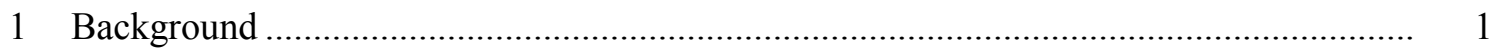

2 Approach: Alternatives to Diesel Displacement ........................................................ 5

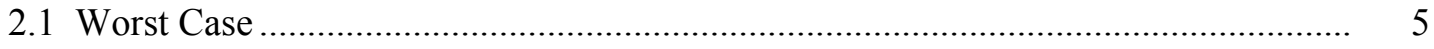

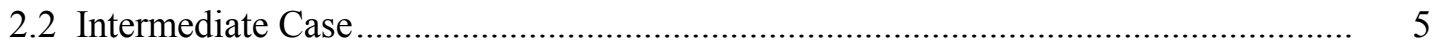

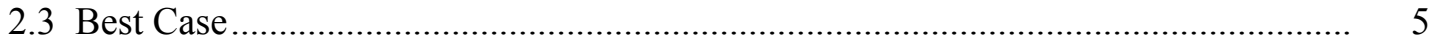

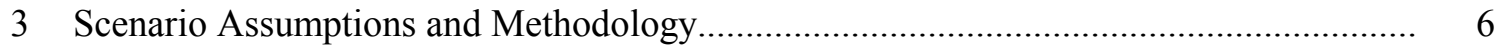

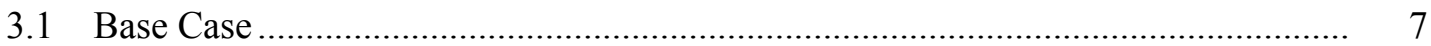

3.1.1 Mobile Sources — Cars and Trucks ............................................................... 9

3.1.2 Mobile Sources — Urban Buses................................................................... 12

3.1.3 Mobile Sources — Off-Road ................................................................. 12

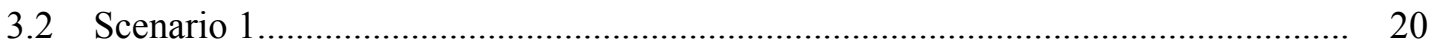

3.2.1 On-Road Vehicles ......................................................................... 20

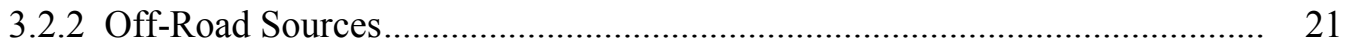

3.2.3 Results for Scenario 1 ........................................................................... 23

3.3 Scenario 2

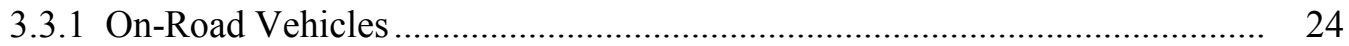

3.3.2 Off-Road Sources............................................................................... 28

3.3.3 Results for Scenario 2 ...................................................................... 30

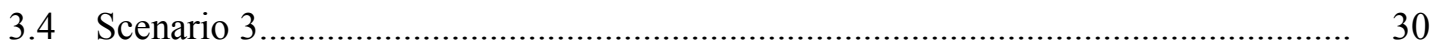

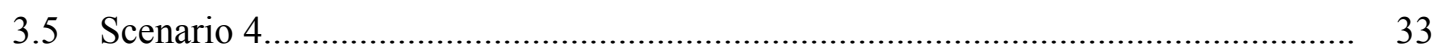

3.6 Changes in Energy Use and Emissions from Production of Alternative Fuels ........ 35

3.7 Changes in Energy Use and Emissions from Production and Combustion of Alternative Fuels ................................................................................... 38

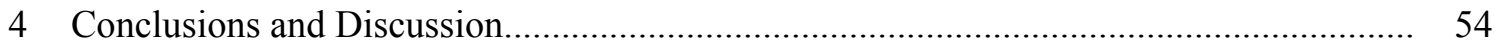

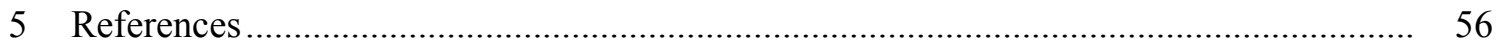




\section{Tables}

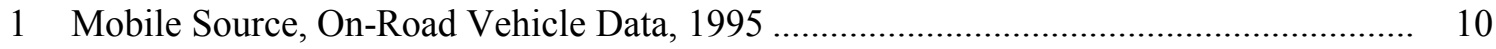

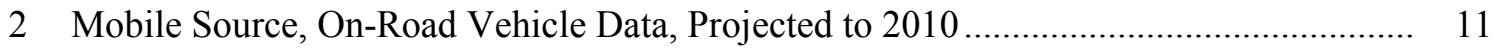

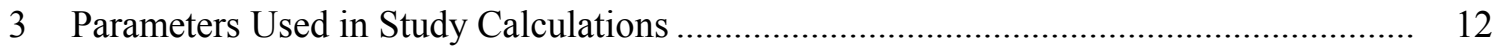

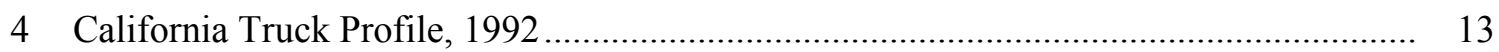

5 California Diesel Truck Profile: VMT vs. Engine Displacement

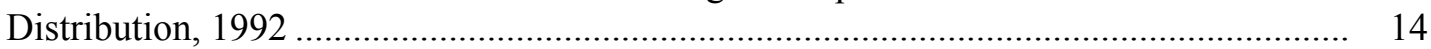

6 Assumptions Regarding California Urban Bus Fleet................................................... 14

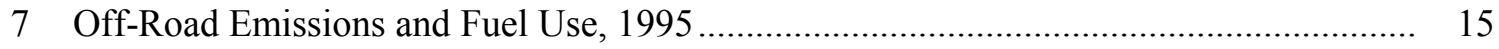

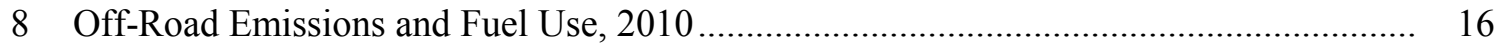

9a Horsepower, Fuel Flow, and Emission Rates by Notch Setting for Unmodified EMD 645 E3B Engine Powered by 100\% Diesel .......................................................... 17

9b Fuel Flow and Emission Rates Weighted by Horsepower and Time in Notch Setting .... 17

10 California Locomotive Fleet, Base Year 1987 ........................................................... 18

11 Factors Used to Calculate Emission Changes for Replacement of Diesel

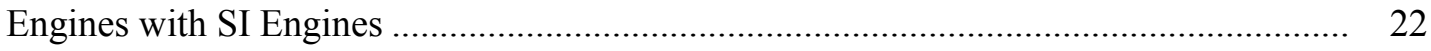

12 Estimated Increases in Energy and Emissions Under Scenario 1, 1995 ......................... 25

13 Estimated Increases in Energy and Emissions Under Scenario 1, 2010 _........................ 26

14 Fuel Use and Emission Rates for Heavy-Duty Vehicles Powered by Alternative Fuels

15 Duty-Cycle-Weighted Emission Factors for the Dual-Fuel EMD 16-645

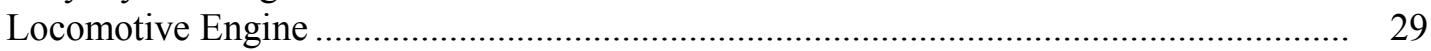

16 Large Diesel and Dual-Fuel Engine Emission Factors at Full Load............................... 31

17 Estimated Increases in Energy and Emissions Under Scenario 2, 2010 ......................... 32

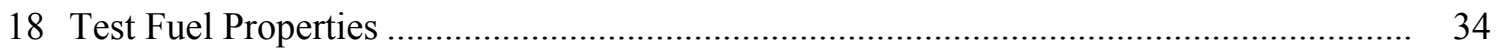

19 Characteristics of Prototype 1991 DDC Series 60 Heavy-Duty Diesel Engine................ 34 


\section{Tables (Cont.)}

20 Summary of Hot-Start Transient Emissions from a Prototype 1991

DDC Series 60

21 Comparisons of Alternative Fuel Properties to California Standards............................. 36

22 Estimated Increases in Energy and Emissions Under Scenario 3, 2010 _........................ 37

23 Combustion-Relevant Properties of DME Compared to Diesel and Other

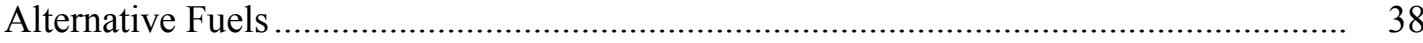

24 Estimated Increases in Energy and Emissions under Scenario 4, 2010 .......................... 39

25 Energy and Emissions from Production of Baseline Diesel Fuel, 2010 .......................... 40

26 Changes in Energy and Emissions Caused by Production of Control Scenario 1

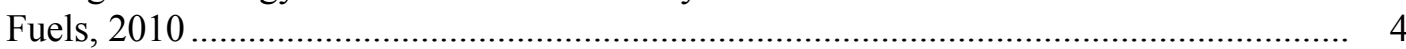

27 Changes in Energy and Emissions Caused by Production of Control Scenario 2

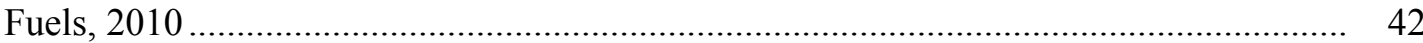

28 Changes in Energy and Emissions Caused by Production of Control Scenario 3 Fuel (FTD), 2010

29 Changes in Energy and Emissions Caused by Production of Control Scenario 4 Fuel (DME), 2010

30 Net Changes in Energy and Emissions Caused by Production and Combustion of Control Scenario 1 Fuels, 2010

31 Net Changes in Energy and Emissions Caused by Production and Combustion of Control Scenario 2 Fuels, 2010.

32 Net Changes in Energy and Emissions Caused by Production and Combustion of Control Scenario 3 Fuel (FTD), 2010

33 Net Changes in Energy and Emissions Caused by Production and Combustion of Control Scenario 4 Fuel (DME), 2010.

34 Summary of Energy Increases for the Various Scenarios for 2010 49

35 Summary of $\mathrm{NO}_{\mathrm{x}}$ Emission Changes for the Various Scenarios for 2010 50

36 Summary of $\mathrm{PM}_{10}$ Emission Changes for the Various Scenarios for 2010 52

37 Summary of GHG Emission Changes for the Various Scenarios for 2010 


\section{Notation}

\section{Acronyms and Abbreviations}

\#2D

ANL

APTA

CARB

CBD

$\mathrm{CI}$

$\mathrm{CNG}$

$\mathrm{CO}$

$\mathrm{CO}_{2}$

$\mathrm{COM}$

CTR

DDC

DME

DOE

EF\&EE

EIA

EMD

EPA

FTA

FTD

GE

GHG

GM

GVW

GWP

$\mathrm{HCHO}$

HD

HEI

LCF

LD

LNG

LPG

MD

MHC

$\mathrm{NG}$

$\mathrm{NO}_{\mathrm{x}}$

NMHC

NRDC

NREL

OCTA number 2 diesel

Argonne National Laboratory

American Public Transit Association

California Air Resources Board

central business district

compression ignition

compressed natural gas

carbon monoxide

carbon dioxide

commercial

Center for Transportation Research

Detroit Diesel Corporation

dimethyl ether

U.S. Department of Energy

Engines, Fuel, and Environmental Engineering

Energy Information Administration

Electro Motive Division

U.S. Environmental Protection Agency

Federal Transit Administration

Fischer-Tropsch diesel

General Electric

greenhouse gas

General Motors

gross vehicle weight

global warming potential

formaldehyde

heavy duty

Health Effects Institute

latent cancer fatality

light duty

liquefied natural gas

liquefied petroleum gas

medium duty

methane hydrocarbon

natural gas

oxides of nitrogen

non-methane hydrocarbon

Natural Resources Defense Council

National Renewable Energy Laboratory

Orange County Transit Authority 


$\begin{array}{ll}\text { OHVT } & \text { Office of Heavy Vehicle Technologies } \\ \text { PM } & \text { particulate matter } \\ \mathrm{PM}_{10} & \text { particulate matter measuring less than } 10 \text { microns } \\ \mathrm{PM}_{2.5} & \text { particulate matter measuring less than } 2.5 \text { microns } \\ \mathrm{PRF} & \text { pseudo-CARB reference fuel } \\ \text { QRA } & \text { quantitative risk assessment } \\ \text { ROG } & \text { reactive organic gas } \\ \mathrm{SI} & \text { spark ignition } \\ \mathrm{SO}_{2} & \text { sulfur dioxide } \\ \mathrm{SO}_{\mathrm{x}} & \text { oxides of sulfur } \\ \mathrm{SRP} & \text { Scientific Review Panel } \\ \text { THC } & \text { total hydrocarbon } \\ \text { TIUS } & \text { Truck Inventory and Use Survey } \\ \mathrm{ULEV} & \text { ultra-low emission vehicle } \\ \mathrm{UWV} & \text { University of West Virginia } \\ \text { VMT } & \text { vehicle miles traveled }\end{array}$

\section{Units of Measure}

$\begin{array}{ll}\text { atm } & \text { atmosphere } \\ \text { bhp-h } & \text { brake-horsepower hour } \\ { }^{\circ} \mathrm{C} & \text { degrees Celsius } \\ \mathrm{d} & \text { day } \\ \mathrm{ft} & \text { foot } \\ \mathrm{g} & \text { gram } \\ \text { gal } & \text { gallon } \\ \mathrm{GJ} & \text { gigajoules } \\ \mathrm{h} & \text { hour } \\ \mathrm{hp} & \text { horsepower } \\ \mathrm{kWh} & \text { kilowatt hour } \\ \mathrm{L} & \text { liter } \\ \mathrm{lb} & \text { pound } \\ \mathrm{MJ} & \text { megajoules } \\ \mathrm{rpm} & \text { rotations per minute } \\ \text { scf } & \text { standard cubic foot }\end{array}$

\section{Terms and Variables Used in Equations}

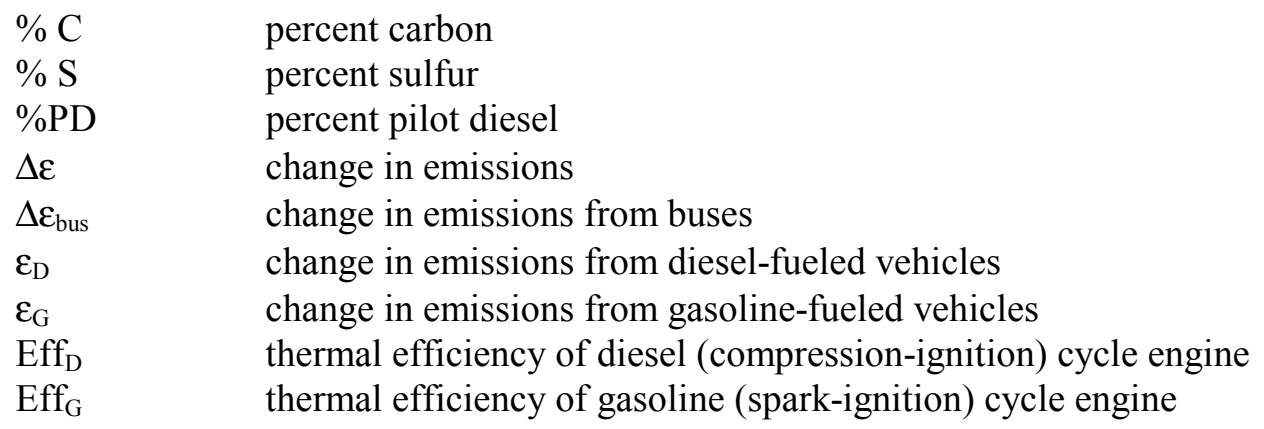


Eff $_{\mathrm{NG}}$

F

fbuses

fbuses $_{\leq 27.5 \mathrm{ft}}$

fbuses $>27.5 \mathrm{ft}$

$\mathrm{F}_{\mathrm{D}}$

$\mathrm{F}_{\text {DME }}$

$\mathrm{F}_{\text {Dreplace }}$

$\mathrm{FE}_{10-16 k}$ lb buses

$\mathrm{FE}_{14-16 \mathrm{k} \text { lb G trucks }}$

$\mathrm{FE}_{\mathrm{D}}$

$\mathrm{FE}_{\text {Dbuses }}$

$\mathrm{FE}_{\mathrm{G}}$

$\mathrm{FE}_{\mathrm{HDD}}$

FE $E_{\text {HDGtrucks }}$

$\mathrm{FE}_{\mathrm{NG} \text { buses }}$

$\mathrm{F}_{\text {FT }}$

$\mathrm{F}_{\mathrm{G}}$

$\mathrm{F}_{\mathrm{NG}}$

$\mathrm{F}_{\mathrm{PD}}$

$\mathrm{HDG}_{\text {equip }}$

$\mathrm{LDG}_{\text {equip }}$

$\mathrm{LHV}_{\mathrm{D}}$

$\mathrm{LHV}_{\mathrm{DME}}$

$\mathrm{LHV}_{\mathrm{FT}}$

$\mathrm{LHV}_{\mathrm{G}}$

Max

Min

$\mathrm{VMT}_{\leq 8 \mathrm{~L}}$

$\mathrm{VMT}_{>8 \mathrm{~L}}$

$\mathrm{VMT}_{\mathrm{D}}$

$\mathrm{VMT}_{\text {Dbuses }}$

$\mathrm{VMT}_{\text {Dreplace }}$

$\mathrm{VMT}_{\mathrm{G}}$

$\mathrm{VMT}_{\text {total }}$

$\mathrm{X}_{\mathrm{DG}}$ thermal efficiency of natural gas (spark-ignition or pilot diesel compressionignition) engine

fuel (consumed)

fraction of buses (having a given property)

fraction of buses in California fleets that are less than or equal to 27.5 feet in length

fraction of buses in California fleets that are greater than 27.5 feet in length

fuel demand by diesel-fueled vehicles

fuel demand by dimethyl ether-fueled vehicles

fuel demand by vehicles replacing diesel-fueled units

fuel efficiency of buses weighing 10,000-16,000 pounds

fuel efficiency of gasoline-fueled trucks weighing 14,000-16,000 pounds

fuel efficiency (travel distance per unit fuel consumption) of diesel-fueled vehicles

fuel efficiency (travel distance per unit fuel consumption) of diesel-fueled buses

fuel efficiency (travel distance per unit fuel consumption) of gasoline-fueled vehicles

fuel efficiency (travel distance per unit fuel consumption) of heavy-duty dieselfueled vehicles

fuel efficiency (travel distance per unit fuel consumption) of heavy-duty gasoline-fueled trucks

fuel efficiency (travel distance per unit fuel consumption) of natural gas-fueled vehicles

Fischer-Tropsch (synthetic) fuel consumed

gasoline consumed

natural gas consumed

diesel consumed as a pilot fuel in natural gas engines

heavy-duty (off-road) equipment powered by gasoline

light-duty (off-road) equipment powered by gasoline

lower heating value (Btu or joules) of diesel fuel

lower heating value (Btu or joules) of dimethyl ether fuel

lower heating value (Btu or joules) of Fischer-Tropsch synthetic diesel

lower heating value (Btu or joules) of gasoline

maximum (highest) value from a choice of values

minimum (lowest) value from a choice of values

(annual) miles of travel by trucks with engine displacement less than or equal to

\section{8 liters}

(annual) miles of travel by trucks with engine displacement greater than 8 liters

(annual) miles of travel by diesel-fueled vehicles

(annual) miles of travel by diesel-fueled buses

(annual) miles of travel required by substitutes when diesel-fueled buses are replaced

(annual) miles of travel by gasoline-fueled vehicles

(annual) total vehicular miles of travel

diesel to gasoline replacement/equivalency factor (i.e., number of gasoline units require to replace one diesel unit) 


\section{Summary}

The office of Heavy Vehicle Technologies (OHVT), U.S. Department of Energy (DOE), requested that the Center for Transportation Research (CTR) at Argonne National Laboratory (ANL) investigate the options for and consequences of possible future restrictions on the use of diesel fuel in California. In a resolution on August 27, 1998, the California Air Resources Board (CARB) declared that the particulate component of diesel exhaust is a carcinogen and is therefore subject to the public notification provisions of California's Proposition 65. The resolution announced that a risk management process would be undertaken to determine the steps necessary to protect the health and safety of the public from diesel particulate matter (PM) as a toxic air contaminant. These steps could include, but are not necessarily limited to, full implementation of all existing regulations controlling diesel particulate exhaust (from any combustion source), plus selective incremental limitations on sources found to be more detrimental to public health. CARB has declared that its intention is not to ban or restrict diesel fuel, per se, at this time. However, successful lawsuits brought by citizens' organizations and environmental activists' groups have the potential to force CARB's hand beyond its stated intent, which could create a situation of much greater interest (and concern) to DOE. In such a case, the following actions - and consequences - are possible:

1. CARB could issue new specifications requiring diesel fuel reformulation on the basis of more detailed investigation of the exhaust products of individual diesel fuel constituents. This action could increase the penetration of Fischer-Tropsch diesel (FTD) and, eventually, biodiesel and/or dimethyl ether (DME) fuels into the marketplace, although at premium cost and lower full-fuel-cycle efficiency.

2. CARB might outlaw operation of any California-registered on- or off-road vehicle fueled by diesel. This action would increase the penetration of natural gas (most likely in liquefied form), liquified petroleum gas (LPG), and possibly lower alcohols (e.g., methanol, ethanol) into the transportation fuels market, but it also would force some reversion from compression-ignition (CI) to spark-ignition (SI) engines for heavyduty applications.

3. Given the limitations of most substitute fuels, promulgation of (2) could lead to complete elimination of CI engines from some current applications. Partial replacement by gas turbine engines is possible but fraught with problems, such as high fuel consumption at idle. Substitution by SI (Otto) engines would increase fuel consumption because of the lower per-unit hauling capability and less advanced engine technology for heavy-duty service.

From the perspective of optimal use of the most efficient propulsion technologies, ANL has defined two bounding case outcomes ("worst" and "least negative") and an intermediate case outcome, on the basis of the size and composition of the CI engine fleet driven out of operation in California. In the most devastating case ("worst case") with respect to diesel removal 
(Scenario 1), gas turbines would be found unequal to performance requirements in all but a handful of applications, resulting in virtually $100 \%$ replacement of CI by SI engines across the spectrum of light- and heavy-duty vehicles for both on- and off-road use. In Scenario 2, which is not extremely stringent with respect to diesel removal ("intermediate case"), inroads would be made by natural gas and propane in CI heavy-duty truck and locomotive applications; otherwise, the fleet (especially the lighter end) would switch to SI engines. In Scenarios 3 and 4, the least detrimental cases ("best cases"), the CI engine not only survives on such diesel substitutes as FTD or DME, but thrives. Although they are expensive to produce and use per feedstock therm, these substitutes may be acceptable fuels under California regulations because they contain no sulfur or aromatic hydrocarbons.

For each case, ANL examined the magnitude of the changeover in vehicle populations required to reach the desired outcome by the year 2010 and the resulting changes in petroleum energy consumption and emissions. To the extent possible, ANL also evaluated the impacts on atmospheric loading of primary and secondary particulate matter with a diameter of 2.5 microns or less $\left(\mathrm{PM}_{2.5}\right)$ and nitrogen oxides $\left(\mathrm{NO}_{\mathrm{x}}\right)$ that massive shifts to gasoline-, alcohol-, and (potentially) compressed natural gas (CNG)-fueled SI engines would be likely to produce.

For the worst case, in which conventional diesel fuel would be banned and gas turbines would have only limited applications, the number of medium- and heavy-duty trucks, in particular, would have to increase by as much as $50 \%$ to accommodate projected requirements for road freight tonnage. All of these trucks would be gasoline-fueled, and the incremental daily demand for gasoline by trucks in California (with diesel demand zeroed out) would be about 15 million gallons, or 350,000 barrels. This amount is just less than half of California's current daily gasoline consumption - about $37 \%$ of projected year-2010 daily gasoline demand (absent the diesel prohibition). This estimate does not include the additional seven million gallons required by other vehicle types and in "upstream" fuel production and transport activities. An overall daily diversion of this magnitude (over 1/2 million barrels) from normal refinery production uses could impose significant short-term stress on domestic refiners and lead to spot shortages elsewhere in the United States. California motorists would also likely have to pay a substantial premium for the diverted gasoline. Not surprisingly, this scenario would cause a large increase in mass emissions of carbon monoxide (CO) and greenhouse gases (GHGs). Daily demand for liquefied natural gas (LNG) as a transportation and process fuel would also increase from a negligible level today to 4.5 million gallons (60,000 diesel-equivalent barrels) in 2010 .

In the intermediate case, diesel pilot fuel would be acceptable for use in natural gas (NG)powered heavy-duty vehicles. Gasoline demand in California would increase by 32.2 million liters per day overall — about $21 \%$ above California's baseline projections. Natural gas demand would increase by 13.6 million diesel-liter equivalents (lower heating value) per day. This increased demand for NG, an incremental 5.4 million standard cubic meters, represents an increase of about $7 \%$ relative to projected statewide consumption. End-use energy consumption and air emissions changes would include increases in $\mathrm{CO}$ and reactive organic gas (ROG) (relative to baseline projections) from engines that shift from CI to gasoline-fueled SI (e.g., refrigeration units, many medium-duty trucks), even when catalytic control is assumed. Sulfur oxides $\left(\mathrm{SO}_{\mathrm{x}}\right)$ and particle matter with a diameter of 10 microns or less $\left(\mathrm{PM}_{10}\right)$ would be reduced relative to the base case, but $\mathrm{NO}_{\mathrm{x}}$ reduction would be less dramatic. On a full-fuel-cycle 
basis, CO and global warming potential (GWP)-weighted GHG emissions would increase less over baseline values than in the diesel removal case (Scenario 1), although methane emissions would increase as a result of greater NG consumption. $\mathrm{SO}_{\mathrm{x}}$ and $\mathrm{PM}_{10}$ would be reduced by a greater amount than in Scenario 1, but $\mathrm{NO}_{\mathrm{x}}$ reduction would be less dramatic.

The case involving replacement of conventional diesel with Fischer-Tropsch synthetic diesel made from NG (Scenario 3) would result in a demand for FTD of almost 76 million liters per day. This total, which includes use of FTD as a replacement process fuel, represents an increase of 26.5 million diesel-equivalent liters over the quantity of diesel displaced. Using DME (Scenario 4) would require somewhat less diesel-equivalent energy to replace diesel fuel (64 million liters - an increase of 14.5 million diesel-equivalent liters). Over the full fuel cycle, emissions of priority pollutants would decline for Scenario 3, while GHG emissions could rise by an amount intermediate to the other diesel replacement scenarios. Reduction in $\mathrm{SO}_{\mathrm{x}}$ emissions would be especially dramatic; reduction of fine particulates would be less so. Scenario 4 requires somewhat less diesel-equivalent energy for replacement fuel (17 million gallons) and results in smaller increases in GHG emissions and greater decreases in $\mathrm{PM}_{10}$ and $\mathrm{NO}_{\mathrm{x}}$ emissions than does Scenario 3, but DME actually increases CO relative to baseline values because of the oxygen in the fuel.

No single scenario yields the least combined impact for all of the important components of emissions and energy use. While Scenario 1, which essentially eliminates CI propulsion engines, would probably be the most straightforward to implement, it would also consume the greatest amount of energy. Use of FTD (Scenario 3) would also be quite easy to implement, but it too would consume a large amount of energy. Scenario 2, which retains CI but uses a substantial amount of NG in SI applications, may be the best choice to minimize overall negative effects on energy and the environment (not just those in California), but it would still require an extraordinary effort in vehicle and engine replacement over the next 10 years.

It is important to note that this study is not a cost/benefit analysis. Dollar value trade-offs for damages avoided by reducing particulate, $\mathrm{NO}_{\mathrm{x}}$, and $\mathrm{SO}_{\mathrm{x}}$ emissions (net benefits) have not been compared to general increases in energy use, GHGs, and other criteria pollutants. Similarly, the net benefits revealed by such comparisons, if any, would have to be shown to exceed the costs of making the changes in fuels and vehicles for each scenario. Such analyses were beyond the scope of this study. 
$\Delta$ 


\section{Section 1 Background}

On April 24, 1998, the California Scientific Review Panel (SRP), a nine-member body of distinguished scientists comparable in function and authority to the President's Science Advisory Board at the federal level, concurred with an earlier decision by the California Air Resources Board (CARB) that whole diesel exhaust is a carcinogen and recommended that it be considered a toxic air contaminant under California state standards (Inside EPA Mobile Source Report [MSR] 1998a). Stating that diesel exhaust is "...the most important public health issue (the panel) has ever addressed," the SRP estimated that as many as 14,850 California residents could ultimately die of cancer as a direct consequence of exposure to diesel. SRP also maintained that a lifetime of exposure to diesel at concentrations typical of those in ambient air in many California locations would cause some 450 incidents of lung cancer per million people exposed.

This finding immediately unleashed a storm of protest from both the petroleum production and heavy-duty vehicle communities; the California Trucking Association went so far as to refer to the data on which SRP's decision was based as "junk science" (MSR 1998a). The concern of these organizations was justified because CARB generally acts in accordance with SRP's recommendations. However, after deliberations carrying over from its July to its August 1998 meeting, CARB voted to limit its declaration of diesel exhaust toxicity to the particulate matter component (CARB 1998a). This limited declaration has several interesting ramifications that we discuss later.

At the heart of the controversy surrounding the potential dangers of diesel combustion exhaust is the Technical Support Document on diesel toxicity that CARB re-released (in revised form) in February 1998 (CARB 1998b). This document provides a technical basis for CARB's estimates and projections of indoor and outdoor exposures to the components of diesel exhaust in the state's populated areas. However, the tone is much more obviously cautious than that of the preceding report released in May 1997 (CARB 1997a). In determining average exposures in California's most densely populated areas, CARB used indigenous source-apportioned measurements of particulate matter with a diameter of 10 microns or less $\left(\mathrm{PM}_{10}\right)$ at local monitors, but the report authors were careful to enumerate the data extrapolation assumptions for areas where no such measurements were taken. Extrapolation was used to estimate total population exposures on the basis of weighted proportions from each source category. The most important assumptions (and limitations) are listed below.

- Virtually all extrapolations are based on data from monitors located near population centers where there are diesel engine exhaust sources. Although researchers attempted to account for this weakness, it is possible that estimates developed for more sparsely populated areas may be too high.

- The inventory that served as the basis for extrapolation was not speciated, and chemical mass balance was not applied at any of the monitors from which the inventory was initially developed. Both CARB and various air management districts around the state 
have documented many uncertainties associated with both the 1990 and $1995 \mathrm{PM}_{10}$ inventories, although fewer concerns are associated with the 1995 study.

- The inventory was developed from 24-h average measurements. To more accurately associate ambient readings and intensities with activity and sources, hourly samples should have been collected.

- The extensive list of "identified or tentatively identified" species and species groups in diesel exhaust does not provide a percentage contribution for any of the known or suspected carcinogens in the list. Further, the sources of most of the information are ten years old or more.

- Neither CARB nor the U.S. Environmental Protection Agency (EPA) defines a diesel exhaust exposure threshold above which carcinogenesis becomes likely. As with inhalation exposure to lead, the threshold may be effectively zero. However, if there is a non-zero threshold, the clear evidence is that average exposures to diesel particulate matter (PM) exhaust in California are moving downward toward it; PM levels declined more than 25\% from 1990 to 1996 . This trend will continue (at least through 2010) with further improvements in diesel emission controls and with expanding use of California reformulated diesel fuel, eventually reaching a level only about $15 \%$ of the 1990 level.

The question of whether chronic exposure to low concentrations or acute exposure to high concentrations of fine PM is more dangerous to human beings and, in particular, to sensitive populations has not been answered. Anecdotal evidence from past high-exposure episodes (such as the Donora, Pennsylvania, and London, England, killer smog episodes) suggests that acute exposure may be more harmful. Carcinogenic potential, however, is dimensionless: quantity is completely overtaken by quality. Thus, EPA's own recent conclusion that diesel exhaust is a probable human carcinogen [MSR 1998b] does not include human exposure levels and does not rely on the rat exposure data that were used as part of the basis of an earlier study. Interestingly, EPA's report also does not include an estimate of the latent cancer fatality (LCF) rate, but it does indicate that risks are spread over a broad range.

Both California and EPA have relied chiefly on data from epidemiological studies, many of which are themselves controversial. In particular, a signal study of exposure to diesel exhaust by U.S. railroad workers (Garshick et al. 1998) that has served as a source of much inferential information was recently determined by an expert panel of the Health Effects Institute (HEI) to be too limited in scope and structure to provide a reliable basis for quantitative risk assessment (QRA) (HEI 1999). Another study of diesel exposure and cancer incidence among teamsters (Steenland et al. 1998) was judged by the HEI panel to be more suitable for QRA because of its greater relevance to ambient exposures by the general public; however, further evaluation of this relatively recent study is required before researchers can judge its suitability for QRA. In Europe, where average human exposure to highway diesel exhaust tends to be higher because of a higher number of light-duty diesel vehicles, effects studies are similarly inconclusive because not all confounding effects (e.g., smoking; "second-hand" exposure to indoor PM, including coal, fuel oil, and tobacco combustion particles) were uniformly controlled. 
Environmental interest groups have been quick to respond to the recent studies. In a set of lawsuits filed April 28, 1998, in the Superior Court of California, the Natural Resources Defense Council (NRDC), and California Attorney General Dan Lundgren charged supermarket chains Safeway, Inc., Lucky Stores, Stater Brothers, and Ralph's Grocery Company with "knowingly and willfully exposing individuals to carcinogenic diesel exhaust" without first providing a clear and reasonable warning about exposure to chemicals that may cause cancer, as required by California Proposition 65 (MSR 1998a). The chief complainants in the case are residents near large warehousing facilities operated by the grocery store chains, where heavy diesel truck activity and idling occurs throughout the day and night. Whether these residents occupied their homes before or after construction of the warehouse facilities is not relevant under California law, because the burden of information disclosure under Proposition 65 remains with the "polluting" facility. The environmental community has made public its objective in this case - to force the supermarket chains to operate a higher proportion of their fleets on natural gas (NG) - although they realize that they will not remove diesel trucks from the road by this or any other maneuver in the near term. The most noteworthy aspect of this proceeding is the state's direct participation on the side of the plaintiffs, a clear indication of its policy.

The Office of Heavy Vehicle Technologies (OHVT), U.S. Department of Energy (DOE), requested that the Center for Transportation Research (CTR) at Argonne National Laboratory investigate the options for and consequences of possible future restrictions on the use of diesel fuel in California. As discussed above, many uncertainties remain in the implication of diesel exhaust as a carcinogen. In its 1998 resolution, CARB stated that a risk management process would be undertaken to determine exactly what steps are necessary to protect the public health and safety from diesel PM as a toxic air contaminant (CARB 1998a). These steps may include, but are not limited to, full implementation of all existing regulations controlling diesel particulate exhaust (from any combustion source), plus selective incremental limitations on sources found to be more detrimental to public health. Meanwhile, though, possibly as a means to defuse (at least temporarily) the lawsuit fervor caused by the April 28, 1998, filings, the Board stipulated that the introduction of risk factors (i.e., rates of latent cancer incidence per microgram per cubic meter of ambient particulate concentration) into civil litigation proceedings would require that "...the proper courts... determine whether the use of the reported unit risk factors is appropriate for the specific sources, fuel formulations, engine technologies, and exposures at issue in any such litigation." (emphasis added) (CARB 1998b).

CARB has declared that its intention is not to ban or restrict diesel fuel, per se, at this time. However, at present, only (largely voluntary) good will efforts are under way by the grocery chains and other fleet operators to accelerate the transition from diesel to (predominantly) liquefied NG as a heavy truck fuel. If these efforts falter, more lawsuits may force CARB's hand beyond its original intent, which could create a situation of much greater interest (and concern) to DOE. In such a case, the following actions - and consequences - are possible:

1. CARB could issue new specifications requiring diesel fuel reformulation on the basis of more detailed investigation of the exhaust products of individual diesel fuel constituents. This action could increase the penetration of Fischer-Tropsch diesel (FTD) and, eventually, biodiesel and dimethyl ether (DME) fuels into the marketplace, although at premium cost and lower full-fuel-cycle efficiency. 
2. CARB could outlaw operation of any California-registered on- or off-road vehicle powered by diesel fuel. This action would increase the penetration of NG (most likely in liquefied form), liquified petroleum gas (LPG), and possibly lower alcohols into the transportation fuels market, but it would force some reversion to spark-ignition (SI) engines for heavy-duty applications.

3. Given the limitations of most substitute fuels, promulgation of action (2) could lead to complete elimination of compression-ignition (CI) engines from some current applications. Partial replacement by gas turbine engines is possible but fraught with problems, such as high fuel consumption at idle. Substitution of CI engines by SI (Otto) engines would increase fuel consumption because of the lower per-unit hauling capability and less advanced engine technology for heavy-duty service. 


\section{Section 2 \\ Approach: Alternatives to Diesel Displacement}

Each of alternatives 1 through 3, outlined in Section 1, would result in some diesel displacement scenario, with variations on either side. Because the specific findings and resulting regulations to control diesel emissions in California were not specified for this study, we needed to define several strategies to encompass the full range of potential regulations. From the perspective of optimal use of the most efficient propulsion technologies, we defined bounding cases ("worst" and "best" [or least negative]) and an intermediate case on the basis of the size and composition of the diesel-fueled, CI engine fleet driven out of operation in California.

\subsection{Worst Case}

In the most devastating case with respect to diesel removal, gas turbines would be found unequal to performance requirements in all but a handful of applications, resulting in virtually $100 \%$ replacement of CI engines by SI engines across the spectrum of light- and heavy-duty vehicles for both on- and off-road use. For this case, we examine the changeover in vehicle populations required for freight and personal hauling and service trips in California in the year 2010, and the resulting change in petroleum energy consumption and total emissions.

\subsection{Intermediate Case}

In the "not extremely stringent" case with respect to diesel removal, the gas turbine niche market would be somewhat more promising and inroads would be made by NG and propane in CI heavy-duty truck and locomotive applications; otherwise, the fleet (especially the lighter end) would switch to SI engines. Again, we examine the magnitude of changeover in vehicle populations in the year 2010 and the resulting change in petroleum energy consumption and emissions. To the extent possible, we also evaluate the impact on emissions of primary and secondary particulate matter with a diameter of 2.5 microns or less $\left(\mathrm{PM}_{2.5}\right)$ and nitrogen oxides $\left(\mathrm{NO}_{\mathrm{x}}\right)$ that massive shifts to gasoline-, (potentially) compressed natural gas (CNG)-, and alcoholfueled SI engines are likely to produce.

\subsection{Best Case}

In our least detrimental cases, the CI engine would not only survive, but would thrive on diesel substitutes such as FTD or DME which, although expensive to produce and use per feedstock therm, may be acceptable under California regulations. Although, of the four cases, use of FTD or DME would logically cause the least overall disruption to the California economy, there would be costs associated with fuel distribution, replacement fuel production capacity and investment, and total energy productivity. However, for our analysis, we do not attempt to quantify these costs. 


\section{Section 3 \\ Scenario Assumptions and Methodology}

Four scenarios have been developed to cover the cases described in Section 2. In each case, the scenario's outcomes must be indexed to a base case energy use and emissions forecast to the year 2010 for California. The forecast sets the output requirements for all alternative futures departing from this baseline. The base case and four other scenarios (Scenarios 1 through 4) examined in this study are as follows.

- Base Case (1995 and 2010): For the base cases, energy and emissions data are projected from data for 1990-1995 assuming that forecasted activity levels and requirements do not change.

- Scenario 1: Eliminate all CI engines and diesel fuel. Under this scenario, the following changes would occur.

- Light-duty (LD) diesel-powered vehicles would be replaced by gasoline-powered vehicles.

- Every medium-duty (MD) and heavy-duty (HD) truck powered by a diesel engine with a displacement of less than 8 liters (L) would be replaced by a gasoline-fueled truck, while each diesel-fueled truck with a displacement greater than or equal to $8 \mathrm{~L}$ would be replaced by two gasoline-fueled trucks.

- Diesel engines in buses measuring less than 27.5 feet (ft) in length would be replaced by gasoline engines with a displacement of $7.5 \mathrm{~L}$, while diesel-powered buses equal to or greater than $27.5 \mathrm{ft}$ in length would be replaced by 2.7 buses, powered by a gasoline engine with a displacement of $7.5 \mathrm{~L}$.

- Locomotives, vessels, and stationary diesel combustion units would be replaced by hypothetical (i.e., not yet commercially available) SI engines burning NG; technologically, these replacement engines would be similar to the 4,020-horsepower (hp) Caterpillar GenSet ${ }^{\mathrm{TM}}$ engine \#3616.

- All other mobile diesel engine applications would be replaced by gasoline units appropriately de-rated (i.e., rated at a lower horsepower).

- $\quad$ Scenario 2: Eliminate all but pilot diesel fuel and allow CI engines. Under this scenario, the following changes would occur.

- All MD and HD trucks and all buses equal to or greater than $27.5 \mathrm{ft}$ in length would be powered by SI engines fueled with CNG on a 1 for 1 (not 2.7 for 1 ) bus replacement basis; buses less than $27.5 \mathrm{ft}$ in length would retain the gasoline engines described for Scenario 1. A representative truck tractor engine for this 
scenario would be the Caterpillar G3406LE powered by CNG on the commercial (COM) test cycle; a representative bus engine would be the Cummins L10-240G powered by $\mathrm{CNG}$ on the central business district (CBD) test cycle.

- Locomotives and vessels would employ a dual-fuel propulsion system using LNG with ignition pilot diesel, operating under California duty-cycle conditions. A representative engine for this application would be the General Motors (GM) Electro-Motive Division (EMD) 16 645E3B engine modified by Energy Conversions, Inc.

- All other mobile applications would use gasoline engines.

- $\quad$ Scenario 3: Replace all diesel fuel in CI engines with FTD made from NG.

- $\quad$ Scenario 4: Replace all diesel fuel in CI engines with DME made from NG.

\subsection{Base Case}

This section presents the base case energy use and emissions estimates (1995 and 2010) for various mobile sources, including on- and off-road vehicles, and for stationary sources of diesel emissions.

The sources of diesel exhaust emissions addressed in this study comprised all diesel source classes listed in CARB's February 1998 report (CARB 1998b). That report listed annual emissions (1990-1995) of $\mathrm{PM}_{10}, \mathrm{PM}_{2.5}, \mathrm{NO}_{\mathrm{x}}$, oxides of sulfur $\left(\mathrm{SO}_{\mathrm{x}}\right)$, carbon monoxide (CO), and reactive organic gases (ROG) for each of the source categories listed below. The source of these data was cited as CARB's 1990 and 1995 Emissions Data System Estimates for Mobile and Stationary Sources for California (January 2, 1998).

- Mobile Sources (diesel only)

$$
\begin{aligned}
& \text { On-Road Vehicles } \\
& \text { LD passenger cars } \\
& \text { LD/MD trucks } \\
& \text { HD trucks } \\
& \text { Urban buses } \\
& \text { Off-Road Vehicles } \\
& \text { Ships } \\
& \text { Trains } \\
& \text { Mobile equipment }
\end{aligned}
$$

- $\quad$ Stationary Area Sources (diesel only)

- Stationary Point Sources (diesel only) 
Because the purpose of the CARB report was quite different from that of the present study, the data contained in it were insufficient for our study in several respects: (1) no energy data were provided; (2) only emissions associated with combustion of the diesel fuel were considered - no estimates of the emissions or energy associated with the production of the fuel were provided; (3) source classes were not broken down in sufficient detail to determine the direct applicability of alternative fuels or engine technologies; and (4) no projections were made of future emissions.

It was necessary, to the extent possible, to find additional sources of emissions and energy data and to resolve any inconsistencies between the data sources. Not all inconsistencies could be resolved and some data were found to be highly suspect. We needed to exercise some judgement in selecting data for this study. The final breakdown of diesel exhaust emission sources and gasoline exhaust emission sources used in our study is given below.

- Mobile Sources (diesel and gasoline vehicles listed separately)

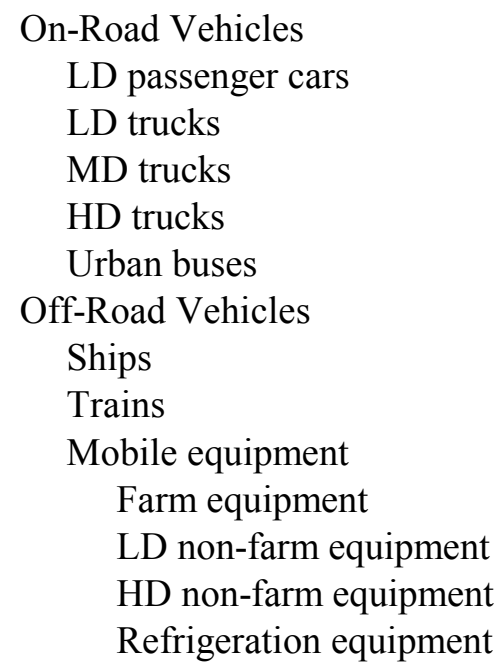

- Stationary Area Sources (diesel only)

- Stationary Point Sources (diesel only)

Each of the source classes listed above is discussed in the following sections. It should be noted that the data used in this study become increasingly uncertain as the discussion progresses through the list of source classes. 


\subsubsection{Mobile Sources - Cars and Trucks}

The base case data for this source category are provided in Tables 1 and 2 for 1995 and 2010, respectively. Most of these data come directly from the statewide totals for the California Vehicle Emissions Ozone Planning Inventory (CARB 1997b), except as follows:

- The breakdown by engine displacement is based on data from the 1992 Truck Inventory and Use Survey (TIUS) database for the State of California (TIUS 1992). The separation by displacement was assumed to be independent of year.

- Carbon dioxide $\left(\mathrm{CO}_{2}\right)$ emissions were calculated by using Equation 1 and the weight percent carbon values listed in Table 3 (Note: terms and variables used in the equations are defined on page vii of the notation list.) The resulting $\mathrm{CO}_{2}$ values are consistent with the limited data given in the California Emission Ozone Planning Inventory (CARB 1997b).

$$
\begin{aligned}
\mathrm{CO}_{2}(\text { tons } / \mathrm{d})=[ & (\% \mathrm{C} / 100 \%) \times 0.5 \times \text { density of fuel }(\mathrm{lb} / \mathrm{gal}) \\
& \times \mathrm{F}(1,000 \mathrm{gal} / \mathrm{d})-0.8 \times \mathrm{ROG}(\text { tons } / \mathrm{d}) \\
& \left.-12 / 28 \times \mathrm{CO}(\text { tons } / \mathrm{d})-\mathrm{PM}_{10}(\text { tons } / \mathrm{d})\right] \times 44 / 12
\end{aligned}
$$

- The sulfur dioxide $\left(\mathrm{SO}_{2}\right)$ emissions were calculated by using Equation 2 and the weight percent sulfur values listed in Table 3 . The resulting $\mathrm{SO}_{2}$ values are consistent with the limited data given in the California Emission Ozone Planning Inventory (CARB 1997b).

$$
\begin{aligned}
\mathrm{SO}_{2}(\text { tons } / \mathrm{d})= & {[(\% \mathrm{~S} / 100 \%) \times 0.5 \times \text { density of fuel }(\mathrm{lb} / \mathrm{gal})} \\
& \left.\times \mathrm{F}(1,000 \text { gal } / \mathrm{d})-0.03 \times \mathrm{PM}_{10}(\text { tons } / \mathrm{d})\right] \times 64 / 32
\end{aligned}
$$

- The $\mathrm{N}_{2} \mathrm{O}$ and $\mathrm{CH}_{4}$ emissions were calculated by using emission factors estimated by Delucchi (1997) and EPA (1998) (the EPA document is in draft form, and the emission factors reported are very preliminary and are believed to be overestimated).

- The fuel economies for the HD gasoline vehicles (with catalysts) and diesel trucks for 1995 and the MD and HD gasoline vehicles (with catalysts) and diesel trucks for 2010 were derived from the TIUS inventory data (TIUS 1992). All other fuel economy values were calculated as the ratio of vehicle miles traveled (VMT) to fuel consumed.

Fuel economy values generally did not show that the diesels operate more efficiently than the gasoline-powered MD and HD trucks. This finding may result from biases in the database; the researchers may have neglected to take into account the differences in loads and driving cycles between gasoline and diesel trucks.

Tables 4 and 5 provide some data on the California truck inventory from the TIUS 1992 database. Note that the population-weighted average fuel economy depends on the type of fuel used, the gross vehicle weight (GVW) of the truck, and the engine displacement. The largest gasoline engines have displacements of less than $8 \mathrm{~L}$. For trucks with GVWs greater than 
Table 1 Mobile Source, On-Road Vehicle Data, 1995

\begin{tabular}{|c|c|c|c|c|c|c|c|c|c|c|c|c|c|c|c|}
\hline \multirow[b]{2}{*}{ Source Type } & \multirow{2}{*}{$\begin{array}{c}\text { Number of } \\
\text { Vehicles } \\
\text { Registered } \\
\left(10^{3}\right) \\
\end{array}$} & \multirow{2}{*}{ 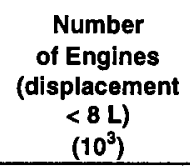 } & \multirow{2}{*}{$\begin{array}{c}\text { Number } \\
\text { of Engines } \\
\text { (displacement } \\
\geq 8 \mathrm{~L}) \\
\left(10^{3}\right)\end{array}$} & \multirow{2}{*}{ 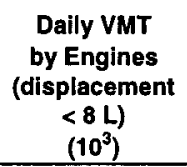 } & \multirow{2}{*}{ 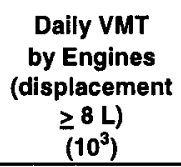 } & \multirow{2}{*}{$\begin{array}{c}\text { Daily Fuel } \\
\text { Use } \\
\left(10^{3} \text { gal }\right) \\
\end{array}$} & \multirow{2}{*}{$\begin{array}{c}\text { Fuel } \\
\text { Economy } \\
\text { (VMT/gal) }\end{array}$} & \multicolumn{8}{|c|}{ Pollutant Emissions $\left(10^{3}\right.$ tons $\left./ \mathrm{d}\right)$} \\
\hline & & & & & & & & ROG & co & $\mathrm{NO}_{x}$ & $\mathrm{CO}_{2}$ & $\mathbf{P M}_{10}$ & so $_{x}$ & $\mathrm{~N}_{2} \mathrm{O}$ & $\mathrm{CH}_{4}$ \\
\hline \multicolumn{16}{|l|}{ Autos } \\
\hline $\begin{array}{l}\text { Gasoline (non catalyst) } \\
\text { Gasoline (catalyst) }\end{array}$ & $\begin{array}{r}958.0 \\
14,106.0\end{array}$ & $\begin{array}{r}958.0 \\
14,106.0\end{array}$ & & $\begin{array}{r}15,700.0 \\
436,100.0\end{array}$ & & $\begin{array}{r}1,291.0 \\
17,954.0\end{array}$ & $\begin{array}{l}12.2 \\
24.3\end{array}$ & $\begin{array}{l}206.0 \\
702.0\end{array}$ & $\begin{array}{l}1,151.0 \\
7,424.0\end{array}$ & $\begin{array}{r}57.8 \\
710.7\end{array}$ & $\begin{array}{r}10.0 \\
159.2\end{array}$ & $\begin{array}{r}0.82 \\
12.20\end{array}$ & $\begin{array}{r}2.64 \\
36.68\end{array}$ & $\begin{array}{r}0.55 \\
130.64\end{array}$ & $\begin{array}{r}3.32 \\
23.05\end{array}$ \\
\hline Auto Total & $\begin{array}{r}180.4 \\
15,244.4\end{array}$ & 180.4 & & $\begin{array}{r}4,230.0 \\
456,030.0\end{array}$ & & $\begin{array}{r}141.0 \\
19,386.0\end{array}$ & 30.0 & $\begin{array}{r}1.9 \\
909.9\end{array}$ & $\begin{array}{r}11.0 \\
8,586.0\end{array}$ & $\begin{array}{r}7.1 \\
775.6\end{array}$ & $\begin{array}{r}1.6 \\
170.8\end{array}$ & $\begin{array}{r}1.52 \\
14.54\end{array}$ & 0.36 & 0.07 & 0.07 \\
\hline \multicolumn{16}{|l|}{ LD Trucks $(<6,000 \mathrm{lb})$} \\
\hline Gasoline (non catalyst) & 359.0 & 359.0 & & $4,700.0$ & & 409.0 & 11.5 & 72.0 & 382.0 & 18.7 & 3.1 & 0.25 & 0.84 & 0.19 & 1.16 \\
\hline Gasoline (catalyst) & $5,882.0$ & $5,882.0$ & & $186,200.0$ & & $8,956.0$ & 20.8 & 351.0 & $3,866.0$ & 493.0 & 79.2 & 5.34 & 18.34 & 77.43 & 11.48 \\
\hline LD Truck Total & $\begin{array}{r}94.7 \\
6,335.7\end{array}$ & 94.7 & & $\begin{array}{r}1,900.0 \\
192,800.0\end{array}$ & & $\begin{array}{r}72.2 \\
9,437.2\end{array}$ & 26.3 & $\begin{array}{r}1.0 \\
424.0\end{array}$ & $\begin{array}{r}5.0 \\
4,253.0\end{array}$ & $\begin{array}{r}3.5 \\
515.2\end{array}$ & $\begin{array}{r}0.8 \\
83.1\end{array}$ & $\begin{array}{l}0.84 \\
6.43\end{array}$ & 0.18 & 0.21 & 0.03 \\
\hline \multicolumn{16}{|l|}{ MD Trucks $(6,000-14,000 \mathrm{lb})$} \\
\hline Gasoline (non catalyst) & 143.0 & 143.0 & & $4,650.0$ & & 804.0 & 5.8 & 16.8 & 277.0 & 35.0 & 7.3 & 1.61 & 1.58 & 2.52 & 1.09 \\
\hline Gasoline (catalyst) & 934.0 & 934.0 & & $36,750.0$ & & $3,723.0$ & 9.9 & 61.0 & 505.0 & 149.0 & 34.9 & 1.78 & 7.65 & 27.26 & 3.56 \\
\hline Diesel MD Truck Total & $\begin{array}{r}162.0 \\
1,239.0\end{array}$ & 118.6 & 43.4 & $\begin{array}{r}4,772.5 \\
46,172.5\end{array}$ & $1,507.5$ & $\begin{array}{l}1,081.0 \\
5,608.0\end{array}$ & 5.8 & $\begin{array}{r}6.0 \\
83.8\end{array}$ & $\begin{array}{r}33.0 \\
815.0\end{array}$ & $\begin{array}{r}42.0 \\
226.0\end{array}$ & $\begin{array}{l}12.2 \\
54.4\end{array}$ & $\begin{array}{l}4.00 \\
7.39\end{array}$ & 3.23 & 0.37 & 0.25 \\
\hline \multicolumn{16}{|l|}{ HD Trucks $(>14,000 \mathrm{lb})$} \\
\hline Gasoline (non catalyst) & 52.0 & 52.0 & & $1,690.0$ & & 297.0 & 5.7 & 10.0 & 190.0 & 20.5 & 2.5 & 0.58 & 0.58 & 1.76 & 0.37 \\
\hline Gasoline (catalyst) & 17.8 & 17.8 & & $1,560.0$ & & 273.0 & 8.5 & 1.8 & 25.0 & 14.0 & 2.6 & 0.13 & 0.56 & 1.67 & 0.21 \\
\hline Diesel & 321.0 & 167.6 & 153.4 & $11,015.8$ & $18,484.2$ & $5,128.0$ & 6.1 & 54.2 & 264.0 & 423.0 & 57.7 & 38.27 & 14.17 & 0.49 & 0.97 \\
\hline HD Truck Total & 390.8 & & & $14,265.8$ & & $5,698.0$ & & 66.0 & 479.0 & 457.5 & 62.8 & 38.98 & & & \\
\hline Diesel Buses & 6.0 & & & 855.0 & & 149.8 & 5.7 & 1.7 & 1.9 & 17.3 & 1.7 & 0.31 & 0.46 & 0.04 & 0.08 \\
\hline Total On-Road Diesel & 764.1 & 561.4 & 196.7 & $22,773.2$ & $19,991.8$ & $6,572.0$ & & 64.8 & 314.9 & 492.9 & 74.0 & 44.94 & 18.41 & 1.18 & 1.41 \\
\hline
\end{tabular}


Table 2 Mobile Source, On-Road Vehicle Data, Projected to 2010

\begin{tabular}{|c|c|c|c|c|c|c|c|c|c|c|c|c|c|c|c|}
\hline \multirow[b]{2}{*}{ Source Type } & \multirow{2}{*}{$\begin{array}{c}\text { Number of } \\
\text { Vehicles } \\
\text { Registered } \\
\left(10^{3}\right) \\
\end{array}$} & \multirow{2}{*}{$\begin{array}{c}\text { Number } \\
\text { of Engines } \\
\text { (displacement } \\
<8 \mathrm{~L} \text { ) } \\
\left(10^{3}\right) \\
\end{array}$} & \multirow{2}{*}{$\begin{array}{l}\text { Number } \\
\text { of Engines } \\
\text { (displacement } \\
\geq 8 \mathrm{~L} \text { ) } \\
\left(10^{3}\right)\end{array}$} & \multirow{2}{*}{$\begin{array}{c}\text { Daily VMT } \\
\text { by Engines } \\
\text { (displacement } \\
<8 \mathrm{~L} \text { ) } \\
\left(10^{3}\right) \\
\end{array}$} & \multirow{2}{*}{$\begin{array}{l}\text { Daily VMT } \\
\text { by Engines } \\
\text { (displacement } \\
\geq 8 \mathrm{~L} \text { ) } \\
\left(10^{3}\right)\end{array}$} & \multirow{2}{*}{$\begin{array}{c}\text { Daily Fuel } \\
\text { Use } \\
\text { (10 gal) } \\
\end{array}$} & \multirow{2}{*}{$\begin{array}{c}\text { Fuel } \\
\text { Economy } \\
\text { (VMT/gal) } \\
\end{array}$} & \multicolumn{8}{|c|}{ Pollutant Emissions $\left(10^{3}\right.$ tons $\left./ \mathrm{d}\right)$} \\
\hline & & & & & & & & ROG & co & $\mathrm{NO}_{\mathbf{x}}$ & $\mathrm{CO}_{2}$ & $\mathrm{PM}_{10}$ & $\mathrm{so}_{\mathrm{x}}$ & $\mathrm{N}_{2} \mathrm{O}$ & $\mathrm{CH}_{4}$ \\
\hline \multicolumn{16}{|l|}{ Autos } \\
\hline $\begin{array}{l}\text { Gasoline (non catalyst) } \\
\text { Gasoline (catalyst) }\end{array}$ & $\begin{array}{c}30.2 \\
18,430.0\end{array}$ & $\begin{array}{c}30.2 \\
18,430.0\end{array}$ & $\begin{array}{l}N^{a} \\
\text { NA }\end{array}$ & $\begin{array}{c}995.0 \\
584,600.0\end{array}$ & $\begin{array}{l}\text { NA } \\
\text { NA }\end{array}$ & $\begin{array}{c}18.5 \\
20,757.0\end{array}$ & $\begin{array}{l}53.7 \\
28.2\end{array}$ & $\begin{array}{c}9.9 \\
235.5\end{array}$ & $\begin{array}{c}71.5 \\
2,771.5\end{array}$ & $\begin{array}{c}3.8 \\
304.2\end{array}$ & $\begin{array}{c}0.0 \\
194.9\end{array}$ & $\begin{array}{c}0.01 \\
15.79\end{array}$ & $\begin{array}{c}0.04 \\
42.31\end{array}$ & $\begin{array}{c}0.04 \\
41.21\end{array}$ & $\begin{array}{c}0.21 \\
30.90\end{array}$ \\
\hline Auto Total & $\begin{array}{c}29.3 \\
18,489.5\end{array}$ & 29.3 & NA & 415.0 & NA & 14.2 & 29.3 & 0.2 & 1.8 & 0.8 & $\begin{array}{c}0.2 \\
195.1\end{array}$ & 0.16 & 0.04 & 0.01 & 0.01 \\
\hline \multicolumn{16}{|l|}{ LD Trucks $(<6,000 \mathrm{lb})$} \\
\hline Gasoline (non catalyst) & 0.0 & 0.0 & NA & 0.0 & NA & 0.0 & NA & 0.0 & 0.0 & 0.0 & 0.0 & 0.00 & 0.00 & 0.00 & 0.00 \\
\hline Gasoline (catalyst) & $8,596.0$ & $8,596.0$ & NA & $277,400.0$ & NA & $12,520.0$ & 22.2 & 89.2 & $1,318.7$ & 218.1 & 118.3 & 7.46 & 25.64 & 28.35 & 14.66 \\
\hline $\begin{array}{l}\text { Diesel } \\
\text { LD Truck Total }\end{array}$ & $\begin{array}{c}6.2 \\
8,602.2\end{array}$ & 6.2 & NA & 88.0 & NA & 3.3 & 26.7 & 0.1 & 0.3 & 0.2 & $\begin{array}{c}0.0 \\
118.3\end{array}$ & 0.04 & 0.01 & 0.00 & 0.00 \\
\hline \multicolumn{16}{|l|}{ MD Trucks $(6,000-14,000 \mathrm{lb})$} \\
\hline Gasoline (non catalyst) & 11.4 & 11.4 & NA & 228.0 & NA & 40.0 & 5.7 & 0.5 & 5.2 & 1.9 & 0.4 & 0.02 & 0.08 & 0.12 & 0.05 \\
\hline Gasoline (catalyst) & $2,043.0$ & $2,043.0$ & NA & $76,110.0$ & NA & $7,138.0$ & 13.1 & 31.5 & 516.7 & 123.5 & 67.9 & 3.41 & 14.67 & 44.53 & 7.04 \\
\hline Diesel MD Truck Total & $\begin{array}{c}342.9 \\
2,397.3\end{array}$ & 251.1 & 91.8 & $8,564.6$ & $2,705.4$ & $1,711.0$ & 14.3 & 1.5 & 57.1 & 38.8 & $\begin{array}{l}19.4 \\
87.6\end{array}$ & 2.86 & 5.32 & 0.37 & 0.38 \\
\hline \multicolumn{16}{|l|}{ HD Trucks $(>14,000 \mathrm{lb})$} \\
\hline Gasoline (non catalyst) & 3.5 & 3.5 & NA & 77.0 & NA & 13.7 & 5.6 & 0.2 & 2.3 & 1.0 & 0.1 & 0.01 & 0.03 & 0.08 & 0.02 \\
\hline Gasoline (catalyst) & 47.0 & 47.0 & NA & $3,026.0$ & NA & 531.0 & 8.5 & 2.2 & 53.8 & 15.4 & 5.0 & 0.26 & 1.09 & 3.23 & 0.40 \\
\hline Diesel $\quad$ HD Truck Total & $\begin{array}{l}427.4 \\
477.9\end{array}$ & 223.2 & 204.2 & $15,138.3$ & $25,401.7$ & $6,135.0$ & 6.1 & 36.5 & 343.3 & 350.9 & $\begin{array}{l}69.1 \\
74.3\end{array}$ & 14.52 & 18.83 & 0.67 & 1.07 \\
\hline Diesel Buses & 6.9 & & & 986.0 & & 150.4 & 6.6 & 1.9 & 1.8 & 15.0 & 1.7 & 0.09 & 0.48 & 0.04 & 0.07 \\
\hline Total On-Road Diesel & 812.7 & 509.8 & 296.0 & $25,191.9$ & $28,107.1$ & $8,013.8$ & & 38.2 & 402.5 & 390.6 & 88.7 & 17.58 & 24.20 & 1.09 & 1.52 \\
\hline
\end{tabular}

a $\mathrm{NA}=$ not applicable. 
Table 3 Parameters Used in Study Calculations

\begin{tabular}{|c|c|c|c|c|c|}
\hline \multirow[b]{2}{*}{ Fuel } & \multirow{2}{*}{$\begin{array}{l}\text { Lower Heating } \\
\text { Value (Btu/gal } \\
\text { or Btu/scf) }\end{array}$} & \multirow{2}{*}{$\begin{array}{c}\text { Density } \\
\text { (liquid: Ib/gal) } \\
\text { (gas: Ib/scf) }\end{array}$} & \multirow{2}{*}{$\begin{array}{l}\text { Fraction } \\
\text { of Carbon }\end{array}$} & \multicolumn{2}{|c|}{ Weight Percentage of Sulfur } \\
\hline & & & & On-Road & Off-Road \\
\hline Gasoline & 115,400 & 6.148 & 0.855 & 0.034 & 0.034 \\
\hline \#2 diesel & 128,700 & 7.137 & 0.870 & 0.045 & 0.390 \\
\hline LNG & 72,900 & 3.500 & 0.740 & 0.003 & 0.003 \\
\hline CNG & 1,031 & 0.045 & 0.740 & 0.003 & 0.003 \\
\hline Methanol & 57,000 & 6.600 & 0.375 & 0 & 0 \\
\hline LPG & 84,000 & 4.405 & 0.740 & 0.003 & 0.003 \\
\hline
\end{tabular}

$19,500 \mathrm{lb}$, the percentage of diesel trucks with engine displacements greater than or equal to $8 \mathrm{~L}$ increases significantly. Also, the percentage of VMT by trucks with the largest diesel engines increases with GVW.

\subsubsection{Mobile Sources — Urban Buses}

Emissions and fuel use data for urban buses in California are shown in Tables 1 and 2. These data are from the California Vehicle Emissions Ozone Planning Inventory (CARB 1997b). On the basis of data from the bus testing program of the Federal Transit Administration (FTA 1997) and recent sales data provided in the American Public Transit Association's (APTA) Transit Fact Book (APTA 1996), we decided that the present bus fleet can be divided into two size classes: vehicles less than or equal to $27.5 \mathrm{ft}$ in length (mostly demand-response [on-call] vehicles) and those longer than $27.5 \mathrm{ft}$ (mostly standard transit buses). Roughly $28 \%$ of the total bus population falls into the first size class, assumed to be powered $50 \%$ by gasoline and $50 \%$ by diesel. The larger size class is assumed to be an all diesel-powered fleet, although some of the uses - depending on the urban area and timeframe - are actually powered by CNG, LNG, or LPG in dedicated- or dual-fuel mode. Urban bus fleet assumptions adopted for this study are summarized in Table 6.

\subsubsection{Mobile Sources - Off-Road}

Vehicle types, emissions, and fuel use data for the off-road diesel and some gasolinepowered sources are shown in Tables 7 and 8 for 1995 and 2010, respectively.

\subsubsection{Locomotives}

The vast majority of locomotives in the United States employ diesel engines to drive electric generators that, in turn, supply electric power to the drive train. The diesel engines range in power rating from about $2,500 \mathrm{hp}$ to the newest 6,000 -hp units. The operating parameters for the diesel engine and electric generator are controlled by throttle positions ("notch" settings) calibrated to maintain near-optimal operating conditions. Fuel use and emission rates are generally measured at each notch setting. Typical duty cycles have been defined for different 
Table 4 California Truck Profile, 1992a

\begin{tabular}{|c|c|c|c|c|c|c|}
\hline \multirow[b]{2}{*}{$\begin{array}{c}\text { GVW Range } \\
\left(10^{3} \mathrm{lb}\right)\end{array}$} & \multirow[b]{2}{*}{$\begin{array}{c}\text { Displacement } \\
\left(\text { in. }{ }^{3}\right)\end{array}$} & \multirow[b]{2}{*}{$\begin{array}{c}\text { Displacement } \\
\text { (L) }\end{array}$} & \multicolumn{2}{|c|}{ Gasoline Trucks } & \multicolumn{2}{|c|}{ Diesel Trucks } \\
\hline & & & $\begin{array}{c}\text { Number } \\
\text { (thousands) }\end{array}$ & $\begin{array}{c}\text { Fuel } \\
\text { Economy } \\
(\mathrm{mpg})\end{array}$ & $\begin{array}{c}\text { Number } \\
\text { (thousands) }\end{array}$ & $\begin{array}{c}\text { Fuel } \\
\text { Economy } \\
\text { (mpg) }\end{array}$ \\
\hline \multirow[t]{6}{*}{$\leq 6$} & $\begin{array}{l}\leq 300 \\
300-399\end{array}$ & $\begin{array}{l}\leq 4.9 \\
4.9-6.5\end{array}$ & $\begin{array}{c}4,244.6 \\
632.6\end{array}$ & $\begin{array}{l}18.6 \\
14.2\end{array}$ & $\begin{array}{l}58.9 \\
11.8\end{array}$ & $\begin{array}{l}17.1 \\
22.9\end{array}$ \\
\hline & $400-499$ & $6.6-8.2$ & 24.6 & 12.3 & 0.0 & NA \\
\hline & $500-599$ & $8.2-9.8$ & 0.0 & $N A^{b}$ & 0.0 & NA \\
\hline & $600-699$ & $9.8-11.5$ & 0.0 & NA & 0.0 & NA \\
\hline & $700-799$ & $11.5-13.1$ & 0.0 & NA & 0.0 & NA \\
\hline & $800-899$ & $13.1-14.7$ & 0.0 & NA & 0.0 & NA \\
\hline \multirow[t]{7}{*}{$6-14$} & $\leq 300$ & $\leq 4.9$ & 172.4 & 13.3 & 3.3 & 5.8 \\
\hline & $300-399$ & $4.9-6.5$ & $1,165.3$ & 13.6 & 49.9 & 17.1 \\
\hline & $400-499$ & $6.6-8.2$ & 275.7 & 12.0 & 61.4 & 12.0 \\
\hline & $500-599$ & $8.2-9.8$ & 0.0 & NA & 0.0 & NA \\
\hline & $600-699$ & $9.8-11.5$ & 0.0 & NA & 0.0 & NA \\
\hline & $700-799$ & $11.5-13.1$ & 0.0 & NA & 0.0 & NA \\
\hline & $800-899$ & $13.1-14.7$ & 0.0 & NA & 0.0 & NA \\
\hline \multirow[t]{7}{*}{$\geq 14$} & $\leq 300$ & $\leq 4.9$ & 18.7 & 12.6 & 86.8 & 6.2 \\
\hline & $300-399$ & $4.9-6.5$ & 44.9 & 8.0 & 20.2 & 7.5 \\
\hline & $400-499$ & $6.6-8.2$ & 95.3 & 8.1 & 38.9 & 7.4 \\
\hline & $500-599$ & $8.2-9.8$ & 0.0 & NA & 13.9 & 5.6 \\
\hline & $600-699$ & $9.8-11.5$ & 0.0 & NA & 24.8 & 6.0 \\
\hline & $700-799$ & $11.5-13.1$ & 0.0 & NA & 3.0 & 5.7 \\
\hline & 800-899 & $13.1-14.7$ & 0.0 & NA & 54.6 & 5.7 \\
\hline $10-14$ & All & NA & 20.8 & 8.7 & 16.6 & 6.6 \\
\hline $14-16$ & All & NA & 11.6 & 9.7 & 8.4 & 7.5 \\
\hline $16-19.5$ & All & NA & 14.1 & 7.6 & 3.1 & 8.3 \\
\hline $19.5-26$ & All & NA & 125.6 & 8.8 & 62.7 & 7.3 \\
\hline $26-33$ & All & NA & 2.9 & 4.2 & 30.3 & 6.6 \\
\hline$>33$ & All & NA & 4.6 & 7.4 & 137.7 & 5.6 \\
\hline \multirow[t]{7}{*}{ Subtotals } & $\leq 300$ & $\leq 4.9$ & $4,435.7$ & NA & 149.0 & NA \\
\hline & $300-399$ & $4.9-6.5$ & $1,842.8$ & NA & 81.9 & NA \\
\hline & $400-499$ & $6.6-8.2$ & 395.6 & NA & 100.3 & NA \\
\hline & $500-599$ & $8.2-9.8$ & 0.0 & NA & 13.9 & NA \\
\hline & $600-699$ & $9.8-11.5$ & 0.0 & NA & 24.8 & NA \\
\hline & $700-799$ & $11.5-13.1$ & 0.0 & NA & 3.0 & NA \\
\hline & $800-899$ & $13.1-14.7$ & 0.0 & NA & 54.6 & NA \\
\hline Grand Total & All & All & $6,673.5$ & & 427.5 & \\
\hline
\end{tabular}

a Values are weighted average fuel economy.

b $\quad$ NA $=$ not applicable. 
Table 5 California Diesel Truck Profile: VMT vs. Engine Displacement Distribution, 1992

\begin{tabular}{|cccc|}
$\begin{array}{c}\text { GVW Range } \\
(\mathbf{1 , 0 0 0} \text { lb) }\end{array}$ & $\begin{array}{c}\text { Displacement } \\
\mathbf{( L )}\end{array}$ & $\begin{array}{c}\text { Diesel } \\
\text { Trucks (\%) }\end{array}$ & $\begin{array}{c}\text { Diesel } \\
\text { VMT (\%) }\end{array}$ \\
\hline$\leq 6$ & $<8$ & 100 & 100 \\
$6-10$ & $<8$ & 100 & 100 \\
$10-14$ & $<8$ & 100 & 100 \\
$14-16$ & $<8$ & 100 & 100 \\
$16-19.5$ & $<8$ & 100 & 100 \\
$19.5-26$ & $<8$ & 74.40 & 53.70 \\
& $>8$ & 25.60 & 46.30 \\
& $>10$ & 11.25 & 27.36 \\
$26-33$ & $<8$ & 47.10 & 27.30 \\
& $>8$ & 52.90 & 72.70 \\
& $>10$ & 41.54 & 54.27 \\
$>33$ & $<8$ & 53.40 & 39.80 \\
& $>8$ & 46.60 & 60.20 \\
& $>10$ & 43.75 & 57.65 \\
\hline
\end{tabular}

Table 6 Assumptions Regarding California Urban Bus Fleet

\begin{tabular}{|ccccc|}
\hline Length (ft) & \% of Fleet & \% Diesel & $\begin{array}{c}\text { Representative } \\
\text { Gasoline Engine } \\
\text { Displacement } \\
\text { Range (L) }\end{array}$ & $\begin{array}{c}\text { Representative } \\
\text { Diesel Engine } \\
\text { Displacement } \\
\text { Range (L) }\end{array}$ \\
\hline $\begin{array}{c}527.5 \\
>27.5\end{array}$ & 28 & 50 & $7-7.5$ & $5.9-10.4$ \\
& 72 & 100 & & $5.9-10.4$ \\
\hline
\end{tabular}

types of locomotive service. The duty-cycle-weighted average fuel use and emission rates are computed by combining notch-dependent fuel use and emission rates with the time and horsepower at each setting. Tables $9 \mathrm{a}$ and b provide a sample calculation, which applies a California duty cycle defined by Engines, Fuels, and Environmental Engineering, Inc. (EF\&EE 1993). According to a study performed for CARB by EF\&EE, the composition of the California locomotive fleet in 1987 was as shown in Table 10.

The locomotive fuel use shown in Table 10 was increased for 1995 and 2010 to 598,000 and $792,000 \mathrm{gal} / \mathrm{d}$, respectively, in response to recent data on diesel fuel sales to railroads (DOE and EIA 1996). The emissions were left unchanged in accordance with estimates made by EF\&EE (1993) and CARB (1997a,b), which assume that improvements in emissions 
Table 7 Off-Road Emissions and Fuel Use, 1995

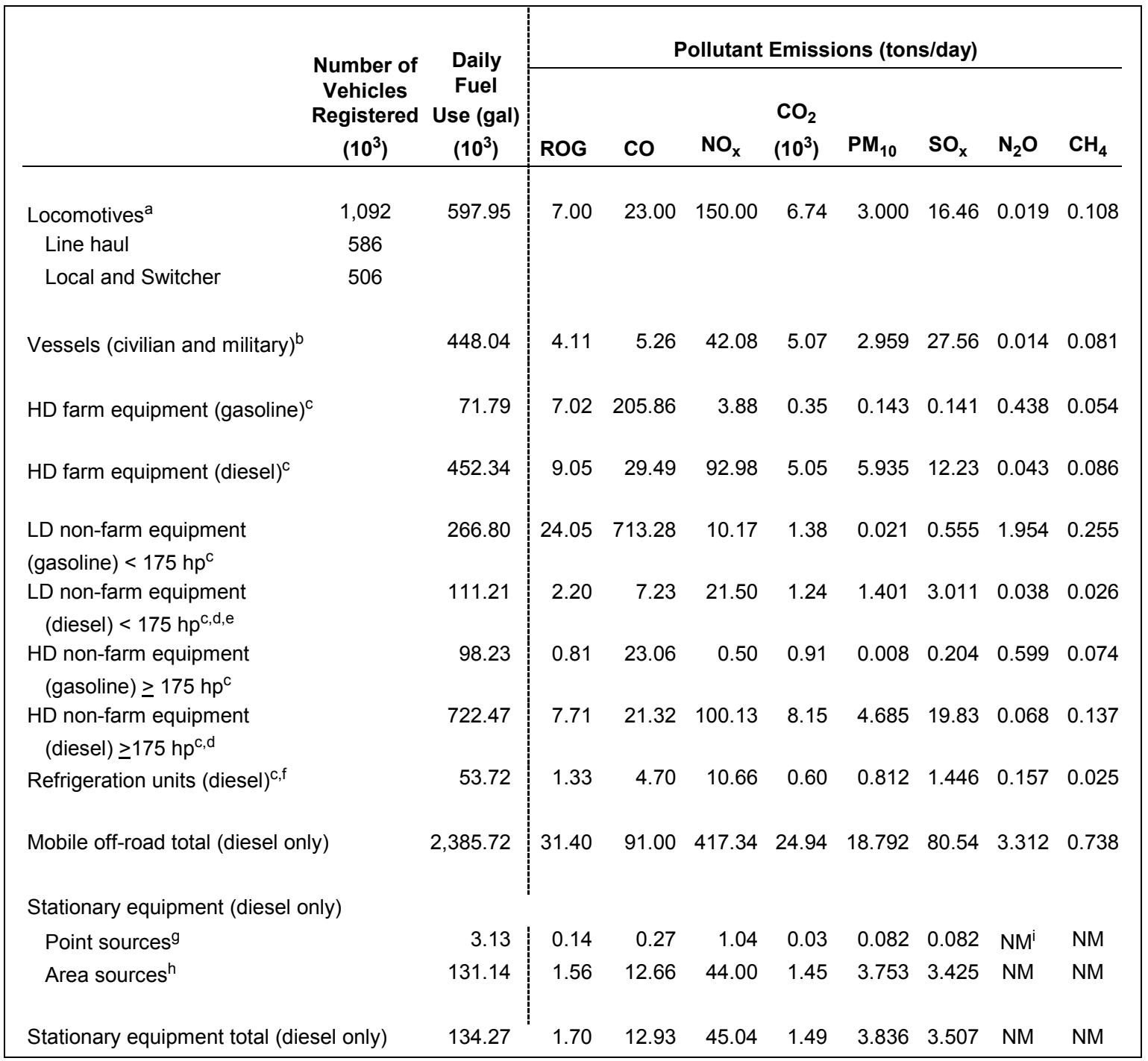

a Locomotive fuel use data are from DOE and EIA 1996; emissions data are from CARB 1998b.

b Fuel use is estimated from diesel fuel sales to commercial vessels and to the military (we assumed all fuel was used for vessels) (fuel data are from DOE and EIA 1996; emissions data and from CARB 1998b).

c Farm equipment data from CARB 1995 for 1990, and growth factors from CARB 1995 for 1990 to 2010 (for diesels only).

d To reconcile with state fuel use data (DOE and EIA 1996), LD and HD fuel use values from CARB were reduced by a factor of 10.

e For consistency with the other sources in the mobile equipment category, emissions for LD non-farm equipment were reduced by a factor of 10 .

$f$ For convenience, refrigeration units are shown at farm equipment rate.

g To estimate diesel fuel used, we assumed point sources equal electric utilities (fuel use data are from DOE and EIA 1996); emissions data are from CARB 1998b).

h Fuel use is estimated from sulfur emissions, assuming area source fuel has the same sulfur content as farm and LD non-farm equipment (emissions data are from CARB 1998b).

i $\mathrm{NM}=$ not measured. 
Table 8 Off-Road Emissions and Fuel Use, 2010

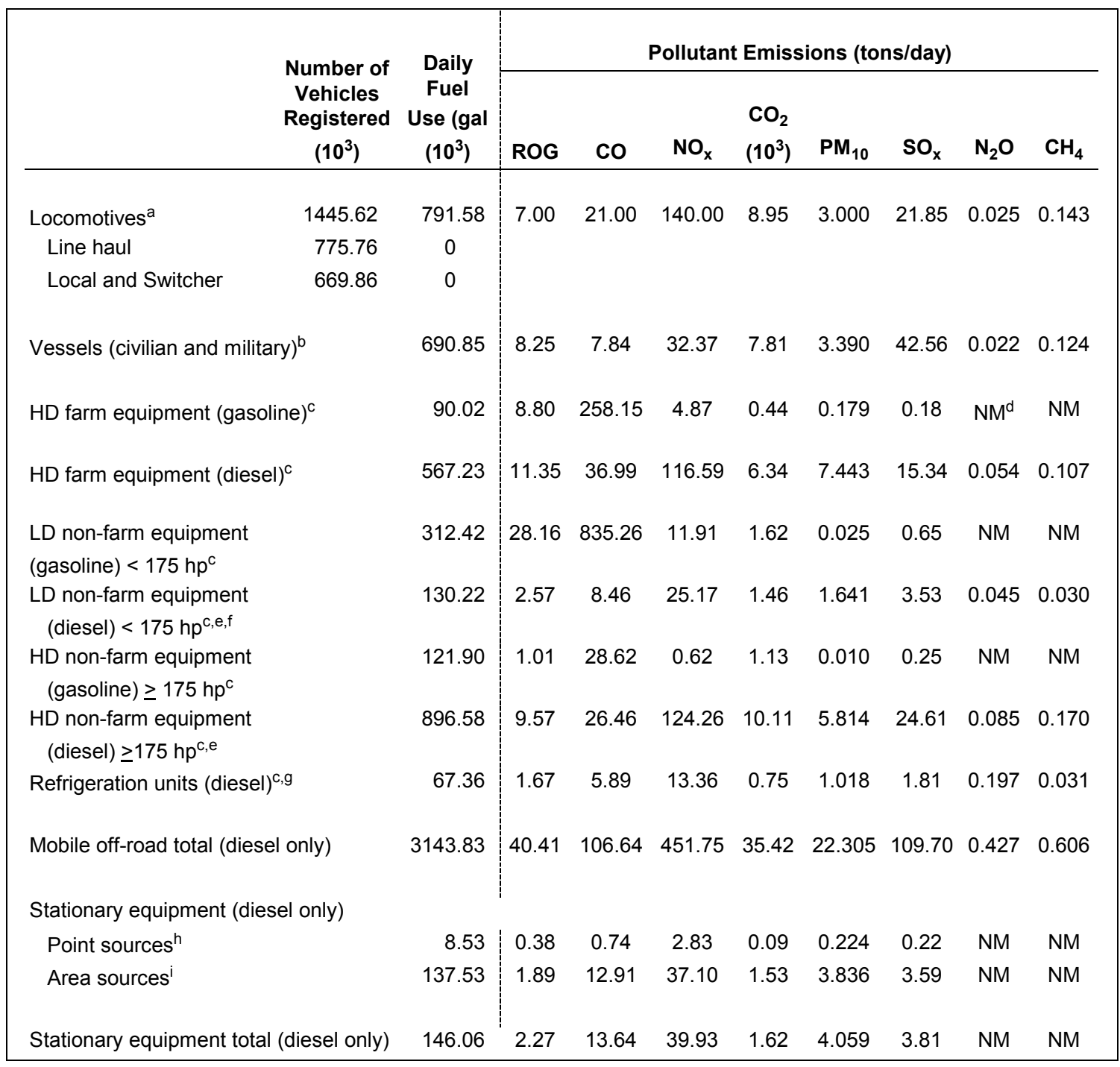

a Locomotive fuel use data are from DOE and EIA 1996; emissions data are from CARB 1998b.

b Fuel use is estimated from diesel fuel sales to commercial vessels and to the military (we assumed all fuel was used for vessels) (fuel data are from DOE and EIA 1996; emissions data are from CARB 1998b).

c Data from CARB 1995 for 1990, and growth factors from CARB 1995 for 1990 to 2010 (for diesels only).

d $\mathrm{NM}=$ not measured.

e To reconcile with state fuel use data (DOE and EIA, 1996), LD and HD fuel use values from CARB were reduced by a factor of 10.

$f$ For consistency with the other sources in the mobile equipment category, emissions for LD non-farm equipment were reduced by a factor of 10 .

$g$ For convenience, refrigeration units are shown at farm equipment rate.

$\mathrm{h}$ To estimate diesel fuel used, we assumed point sources equal electric utilities (fuel use data are from DOE and EIA 1996); emissions data are from CARB 1998b).

i Fuel use is estimated from sulfur emissions, assuming area source fuel has the same sulfur content as farm and LD non-farm equipment (emissions data are from CARB 1998b). 
Table 9a Horsepower, Fuel Flow, and Emission Rates by Notch Setting for Unmodified EMD 645 E3B Engine Powered by $100 \%$ Diesel $^{a, b}$

\begin{tabular}{|c|c|c|c|c|c|c|c|c|c|c|}
\hline \multirow{2}{*}{$\begin{array}{l}\text { Notch } \\
\text { Setting }\end{array}$} & \multirow{2}{*}{$\begin{array}{c}\text { Time in } \\
\text { Setting (\%) }\end{array}$} & \multirow{2}{*}{ Horsepower ${ }^{c}$} & \multirow{2}{*}{$\begin{array}{c}\text { Fuel Flow } \\
\text { Rate } \\
\text { (gal/h) }\end{array}$} & \multirow{2}{*}{$\begin{array}{c}\text { Time-Weighted } \\
\text { Brake- } \\
\text { Horsepower } \\
\text { Hours (bhp-h) }\end{array}$} & \multicolumn{6}{|c|}{ Emission Rates (g/bhp-h) } \\
\hline & & & & & ROG & $\mathrm{CO}$ & $\mathrm{NO}_{\mathrm{x}}$ & $\mathrm{CO}_{2}$ & PM & $\mathrm{SO}_{2}$ \\
\hline Off & 23 & & & 0 & 0 & 0 & 0 & 0 & 0 & 0 \\
\hline Brake & 6.1 & 138 & 7.107 & 8.418 & 5.84 & 12.7 & 48.9 & 556 & 1.35 & 7.91 \\
\hline$I d l e^{C}$ & 39.7 & 241 & 12.412 & 95.677 & 2.65 & 6.35 & 36.1 & 556 & 1.88 & 3.15 \\
\hline 1 & 3 & 312 & 16.069 & 9.360 & 0.84 & 1.8 & 16.5 & 556 & 0.17 & 1.95 \\
\hline 2 & 3.2 & 584 & 30.078 & 18.688 & 0.21 & 0.8 & 11.9 & 423 & 0.3 & 1.48 \\
\hline 3 & 3.1 & 857 & 44.138 & 26.567 & 0.32 & 0.5 & 11.9 & 417 & 0.31 & 1.46 \\
\hline 4 & 3.9 & 1213 & 62.474 & 47.307 & 0.32 & 0.4 & 11.7 & 408 & 0.27 & 1.43 \\
\hline 5 & 3.1 & 1681 & 86.577 & 52.111 & 0.32 & 0.4 & 11.4 & 407 & 0.27 & 1.43 \\
\hline 6 & 2.9 & 2247 & 115.728 & 65.163 & 0.21 & 0.6 & 10.8 & 402 & 0.27 & 1.41 \\
\hline 7 & 2.2 & 3002 & 154.613 & 66.044 & 0.21 & 0.5 & 10.8 & 398 & 0.27 & 1.4 \\
\hline 900 rpm 8 & 9.9 & 3359 & 173.000 & 332.541 & 0.21 & 0.5 & 10.9 & 397 & 0.25 & 1.39 \\
\hline
\end{tabular}

a Notch setting $=$ throttle position.

b Values are for in-line freight services in California.

c Horsepower vs. notch setting data and idle emission data are from McGregor (1994) and Bekken (1994).

Table 9b Fuel Flow and Emission Rates Weighted by Horsepower and Time in Notch Setting

\begin{tabular}{|c|c|c|c|c|c|c|c|}
\hline \multirow[b]{2}{*}{ Notch Setting } & \multirow{2}{*}{$\begin{array}{c}\text { Fuel Flow } \\
\text { Rate (gal/h) }\end{array}$} & \multicolumn{6}{|c|}{ Emission Rates (g) } \\
\hline & & ROG & $\mathrm{CO}$ & $\mathrm{NO}_{\mathbf{x}}$ & $\mathrm{CO}_{2}$ & PM & $\mathrm{SO}_{2}$ \\
\hline Off & 0.000 & 0.000 & 0.000 & 0.000 & 0.000 & 0.000 & 0.000 \\
\hline Brake & 0.434 & 49.162 & 106.909 & 411.640 & 4680.408 & 11.364 & 66.586 \\
\hline Idle & 4.928 & 253.985 & 607.549 & $3,453.940$ & $5,3196.412$ & 180.098 & 301.383 \\
\hline 1 & 0.482 & 7.951 & 16.848 & 154.440 & $5,204.160$ & 1.591 & 18.252 \\
\hline 2 & 0.962 & 3.969 & 14.950 & 222.387 & $7,905.024$ & 5.606 & 27.658 \\
\hline 3 & 1.368 & 8.463 & 13.284 & 316.147 & $11,078.439$ & 8.236 & 38.788 \\
\hline 4 & 2.436 & 15.070 & 18.923 & 553.492 & $19,301.256$ & 12.773 & 67.649 \\
\hline 5 & 2.684 & 16.600 & 20.844 & 594.065 & $21,209.177$ & 14.070 & 74.519 \\
\hline 6 & 3.356 & 13.839 & 39.098 & 703.760 & $26,195.526$ & 17.594 & 91.880 \\
\hline 7 & 3.401 & 14.026 & 33.022 & 713.275 & $26,285.512$ & 17.832 & 92.462 \\
\hline 8 & 17.127 & 70.621 & 166.271 & 3624.697 & $132,018.777$ & 83.135 & 462.232 \\
\hline $\begin{array}{l}\text { Weighted } \\
\text { Average }^{a}\end{array}$ & 37.179 & 0.628 & 1.438 & 14.889 & 425.384 & 0.488 & 1.720 \\
\hline
\end{tabular}

a Weighted averages were calculated using the following formula: $\frac{\sum_{i}(h p \times t i m e)_{i} \sum_{i}}{\sum_{i}(h p \times t i m e)_{i}}$ 
Table 10 California Locomotive Fleet, Base Year 1987

\begin{tabular}{lccrrr}
\hline $\begin{array}{l}\text { Locomotive } \\
\text { Application }\end{array}$ & $\begin{array}{c}\text { Number of } \\
\text { GP60s } \\
\text { (GM/EMD) }\end{array}$ & $\begin{array}{c}\text { Number of } \\
\text { Dash 8s } \\
\text { (GE) }\end{array}$ & $\begin{array}{c}\text { Number of } \\
\text { SD40s } \\
\text { (EMD) }\end{array}$ & $\begin{array}{c}\text { Number of } \\
\text { GP38s } \\
\text { (EMD) }\end{array}$ & Totals \\
\hline Line haul & 70 & 141 & 369 & 0 & 579 \\
Local service & 0 & 0 & 235 & 0 & 235 \\
Switcher & 0 & 0 & 0 & 271 & 271 \\
Fuel use (gal/d) & 57,300 & 113,400 & 329,500 & 39,600 & 540,000 \\
\hline
\end{tabular}

a $\quad \mathrm{GE}=$ General Electric

control technologies will compensate for growth in locomotive populations and fuel use. As an independent check on the locomotive data, we estimated 1995 emissions using duty-cycle emissions factor data for the locomotive types listed above and fuel consumption data from Railroad Facts (1997). Using 7.593 gal/freight-train mile, and assuming an average of three locomotives per train, fuel use and emissions are consistent with the data in Table 7 projected to 1995. The locomotive emissions data for 1995 and 2010 are also very close to the emissions estimates reported on CARB's Internet site for computer runs dated October 17, 1997 (for 1995 data) and July 15, 1997 (for 2010 data).

\subsubsection{Vessels}

Very limited information on marine vessel fuel use, emissions, and vehicle population was available. Estimates of diesel fuel use are based on diesel sales to "vessel bunkering" (which includes sales to commercial and private boats but excludes sales to the military) and "military" in California (DOE and EIA 1996). The 2010 fuel use estimate is based on a linear projection of fuel sales for 1992 and 1996. Emissions data for 1995 were adopted from CARB 1998b. Emissions data for 2010 were estimated from a linear projection of the 1990 and 1995 data contained in CARB 1998b. No independent data sources could be found to validate the CARB vessel data, which do not distinguish between fuel types sold; this is significant because vessels use both distillate and residual fuel oils.

\subsubsection{Mobile Farm Equipment}

Fuel use and emissions data for this source class were available for gasoline and dieselpowered units from CARB (1995), which also provided growth factors for the diesel sources for 1990-2010. The emissions estimates from the 1995 CARB study are essentially the same as those published on CARB's Internet site for computer runs dated October 10, 1997 (for 1995 data) and July 15, 1997 (for 2010 data).

\subsubsection{Mobile Industrial/Commercial Equipment (non-farm equipment)}

Fuel use and emissions data for this source class were available for gasoline and dieselpowered units from CARB 1995, which also provided growth factors for the diesel sources for 
1990-2010. CARB divides this source category into LD (less than $75 \mathrm{hp}$ ) and HD (greater than $75 \mathrm{hp}$ ) equipment.

Consistency checks indicated that CARB's data for this source category were highly suspect. For example, diesel fuel use in the Mobile Industrial/Commercial Equipment category was listed as 8,330,000 gal/d. The fuel sales value from the DOE and EIA (1996) report was $421,000 \mathrm{gal} / \mathrm{d}$ - one twentieth the CARB estimate. If the CARB reference were correct, equipment in this source category would consume more diesel fuel than all on-road vehicles. CARB's overestimate may result from double counting across other source categories, but we have no way to verify this speculation.

We also compared values listed for this source category with those listed for mobile farm equipment and compared ratios of gasoline to diesel fuel for the various source categories; we found that the CARB diesel fuel use figures were much too high. We decided to reduce the CARB diesel fuel use figures for both LD and HD equipment by a factor of ten (i.e., multiply by 0.1). Similar consistency checks of CARB's emissions values, taking into account fuel use ratios, indicated that, while the emissions associated with HD equipment appeared to be consistent with emissions for other source categories, the emissions listed for LD equipment appeared too high (by a factor of 10 or 20) relative to those for HD equipment and those in the farm equipment category. We also decided to reduce the LD equipment emissions estimates by a factor of ten.

The reductions we made in the fuel use and emissions estimates for the LD equipment and in fuel use estimates for the HD equipment categories were intended to bring these source data into concurrence with the other source data for the purposes of this study only. We consider the data for these source categories highly suspect; they should not be used without further investigation in cases where more reliable data are required. ANL analysts brought these data inconsistencies to the attention of CARB staff, who are in the process of updating their emissions inventory, but to date we have received no comment on the suspect data.

\subsubsection{Mobile Refrigeration Equipment}

According to CARB (1995), mobile refrigeration equipment is powered mostly by dieselfueled engines. CARB's fuel use and emissions data for this source class were available for both gasoline and diesel-powered units. Growth factors for the diesel sources were available for 1990-2010. For convenience, the same growth factors were used for this category as for the farm equipment category because they are closely related. No adjustments of the data for this category were made.

\subsubsection{Stationary Point and Area Sources}

Stationary point and area source categories were the least delineated in the literature. Emissions data for both source categories for 1990 and 1995 were taken from CARB 1998b, and projected (linearly) to 2010. Diesel fuel use at point sources was estimated from fuel sales data (DOE and EIA 1996), assuming that point sources and power plants are approximately synonymous. Because no detailed information was available regarding area source populations and types of fuel used, we devised an alternative estimation scheme. Fuel use was estimated from 
sulfur emissions, assuming that the sulfur content of diesel fuel is the same as that used by other off-road sources - namely, farm and LD industrial and commercial equipment (see Table 3).

\subsection{Scenario 1}

\subsubsection{On-Road Vehicles}

In this scenario, every LD diesel-powered highway vehicle would be replaced by catalystcontrolled gasoline-powered vehicles. Fuel use values for this scenario were calculated in two ways, and the higher value was used as the final estimate. In the first method, we used the ratio of miles traveled by diesel vehicles to gasoline fuel economy (see Table 1 for parameter values applied). For the second method, we used the ratio of lower heating values of diesel to those of gasoline multiplied by the ratio of engine efficiencies of diesel to those of gasoline multiplied by the quantity of diesel fuel being displaced, as shown in Equation 3 (see Table 3 for parameter values applied). In general, the second method produced a higher estimate of replacement fuel use.

$$
\mathrm{F}_{\mathrm{G}}=\operatorname{Max}\left(\mathrm{VMT}_{\mathrm{D}} / \mathrm{FE}_{\mathrm{G}}, \mathrm{LHV}_{\mathrm{D}} / \mathrm{LHV}_{\mathrm{G}} \times \mathrm{Eff}_{\mathrm{D}} / \mathrm{Eff}_{\mathrm{G}} \times \mathrm{F}_{\mathrm{D}}\right)
$$

For our analysis (i.e., in the range of effects that can be measured on this scale of engine activity), any potential synergism between the lower heating value and the engine efficiency of diesel was disregarded.

This scenario assumes that MD and HD trucks powered by diesel engines with displacements of less than $8 \mathrm{~L}$ would be replaced by catalyst-controlled gasoline-fueled trucks; those with displacements greater than or equal to $8 \mathrm{~L}$ would be replaced by two catalytically controlled gasoline-fueled trucks, each with an engine displacement of less than $8 \mathrm{~L}$. The 8-L cutoff point was chosen because there are no conventional gasoline-fueled engines with displacements greater than or equal to $8 \mathrm{~L}$.

To calculate fuel use for MD and HD diesel trucks, we had to separate the fleet, fuel use, VMT, and emissions by engine displacement. The fleet VMT breakdown by displacement was estimated on the basis of the TIUS data for California. The replacement gasoline fuel was calculated by using a method similar to that used for the LD vehicles, as shown in Equation 4:

$$
\begin{gathered}
\mathrm{F}_{\mathrm{G}}=\left(\mathrm{VMT} \leq_{8} / \text { total VMT }\right) \times \operatorname{Max}\left(\mathrm{FE}_{\mathrm{D}} / \mathrm{FE}_{\mathrm{G}}, \mathrm{LHV}_{\mathrm{D}} / \mathrm{LHV}_{\mathrm{G}} \times \mathrm{Eff}_{\mathrm{D}} / \mathrm{Eff}_{\mathrm{G}}\right) \times \mathrm{F}_{\mathrm{D}} \\
+\left(\mathrm{VMT}>_{8} / \text { total VMT }\right) \times \mathrm{LHV}_{\mathrm{D}} / \mathrm{LHV}_{\mathrm{G}} \times \mathrm{Eff}_{\mathrm{D}} / \mathrm{Eff}_{\mathrm{G}} \times \mathrm{F}_{\mathrm{D}}
\end{gathered}
$$

In Scenario 1, diesel engines in buses $27.5 \mathrm{ft}$ long or less would be replaced by catalystcontrolled gasoline engines with a displacement of $7.5 \mathrm{~L}$; those greater than $27.5 \mathrm{ft}$ long would be replaced by 2.7 buses powered by catalyst-controlled gasoline engines with a displacement of 7.5 L. The equivalency value of 2.7 results from a comparison of replacement fuel estimated by using two different methods, which in turn depend on the estimated fuel economies of gasoline engines for 14,000 - to $16,000-\mathrm{lb}$ trucks and 26,000 - to $33,000-\mathrm{lb}$ trucks, respectively. These two truck sizes are assumed to have power requirements equivalent to those of the short and long buses (respectively). The following equations were used for this comparison. 
$\mathrm{F}_{\mathrm{G}}=\mathrm{VMT}_{\text {Dreplace }} / \mathrm{FE}_{\mathrm{HDG} \text { trucks }}$

and $\mathrm{F}_{\mathrm{G}}=\left\{\left[\right.\right.$ fbuses $\leq_{27.5 \mathrm{ft}} \times \mathrm{VMT}_{\text {Dbuses }} / \mathrm{FE}_{14-16 \mathrm{k} \mathrm{lb} \mathrm{G} \mathrm{trucks}}+$ fbuses $>27.5 \mathrm{ft} \times \mathrm{VMT}_{\text {Dbuses }} \div$

$\operatorname{Min}\left(\mathrm{FE}_{26-33 \mathrm{k}} \mathrm{lb} \mathrm{G}\right.$ trucks, $\left.\left.\left.\mathrm{LHV}_{\mathrm{G}} / \mathrm{LHV}_{\mathrm{D}} \times \mathrm{Eff}_{\mathrm{G}} / \mathrm{Eff}_{\mathrm{D}} \times \mathrm{FE}_{\mathrm{HDD}}\right)\right]\right\}$,

where $\mathrm{VMT}_{\text {Dreplace }}=\left(\right.$ fbuses $\left.\leq 27.5 \mathrm{ft}_{\text {fbuses }}>27.5 \mathrm{ft} \times \mathrm{X}_{\mathrm{DG}}\right) \times \mathrm{VMT}_{\text {Dbuses }}$

For the parameter values used, both equations yield the same replacement fuel value $(245,000 \mathrm{gal} / \mathrm{d})$ if $\mathrm{X}_{\mathrm{DG}}=2.7$. The change in emissions that results from replacing diesel buses with gasoline buses was computed from Equation 8 using the gasoline VMT and emissions and the diesel emissions listed in Tables 1 and 2 and the diesel VMT replacement value from Equation 7:

$$
\Delta \varepsilon=\left(\varepsilon_{\mathrm{G}} / \mathrm{VMT}_{\mathrm{G}}\right) \times \mathrm{VMT}_{\text {Dreplace }}-\varepsilon_{\mathrm{D}}
$$

\subsubsection{Off-Road Sources}

\subsubsection{Locomotives, Vessels, and Stationary Units}

For Scenario 1, the diesel engines in these three off-road categories would be replaced with hypothetical SI engines burning NG (similar to the 4,020-hp Caterpillar GenSet ${ }^{\mathrm{TM}}$ engine \#3616). We assumed that the NG would be stored as LNG, which affects the production energy and emissions but not the combustion energy and emissions. This option was used in the control scenario because no gasoline engines are available to replace the diesel engines used in these applications.

The options for replacing CI engines in locomotives and vessels are actually limited by both cost and technical factors. Generally, large internal combustion engines are designed for operation within a narrow range of rotations per minute (rpm) and load. They are not readily adaptable to variable-speed applications without substantial modifications or additions (gearboxes, etc.). Diesel electric locomotive drive systems use electric drive components to manage the variable speed requirements. Similarly, other prime movers, such as SI engines or gas turbines, can be combined with electric drive components. Some large vessels, including cruise ships, are now produced with combined diesel/electric drive systems, so their prime movers could also be converted.

For locomotives, electrification of the railway network is a possibility, but would require major infrastructure changes and would be very expensive. Gas turbine engines have been used for high-speed passenger trains ("turbo trains") but they are not considered practical for the current low-speed freight or passenger operations in California. As noted above, gas turbines could be combined with electric drivetrain components to expand their application to locomotives, but this is currently not being done. Examples of possible gas turbine engines used for turbo trains are the Turbomeca model Makila TI 1600, Allied Signal Locomotive Model TF40 (Mull 1995), and Eurodyn's 3,572-hp unit (Davila 1998). All of these gas turbines can burn liquid or gaseous fuels. Gas turbine engines are much lighter, occupy much less space, and 
generate fewer emissions than diesels, but their performance must be de-rated for high altitude, high ambient temperature, and low-load operation.

Another option for locomotives and vessels is the use of large SI engines. Normally, these engines are designed for constant-speed, stationary applications, but they could be adapted to variable-speed applications as described above. However, replacing diesels with SI engines of the same displacement would require that the output of the SI engines be de-rated by $10-50 \%$, depending on the manufacturer and auxiliary equipment (such as turbochargers and air cooling devices) used on the diesel being replaced.

An additional option is the modification of diesel engines to operate in a dual-fuel mode; a small percentage of diesel (5-7\%) would be used as a pilot ignition fuel, but the engine would be powered primarily by NG. Conversion kits are available that make the conversion to dual-fuel use straightforward (Energy Conversions Inc. of Tacoma, Washington, has performed many such conversions for large diesel engines). Burlington Northern Railroad has conducted long-term field tests of a locomotive converted to dual-fuel use with the Energy Conversions Inc. technology; the results have compared favorably with identical but unconverted locomotives in the same freight service. The study did find, though, that operation in low notch settings required $100 \%$ diesel fuel use. As a consequence, the potential benefits in reduced $\mathrm{NO}_{\mathrm{x}}$ and $\mathrm{PM}$ emissions are not as great as expected, and the $\mathrm{CO}$ and total hydrocarbon (THC) emissions were greater. The fuel use penalty was found to be quite small — taking into account the difference in lower heating values of diesel and $\mathrm{NG}$, about $5 \%$ extra fuel was required.

We estimated the changes in emissions associated with replacing large diesel engines with large SI engines burning NG on the basis of a simple comparison of emission factors for the Caterpillar 3616 SI engine powered by NG and typical locomotive diesel engines in the 3,000- to 4,000-hp class operated under full-load (notch 8) conditions. A review of emission factors as a function of notch setting indicates that these factors change relatively little down to notch settings around 4 (i.e., roughly $40-50 \%$ full load). The factors used are listed in Table 11.

On the basis of these data, the change in emissions is equal to change $(\%)+100 \%$ multiplied by the diesel emissions.

Table 11 Factors Used to Calculate Emission Changes for Replacement of Diesel Engines with SI Engines

\begin{tabular}{|lcccc|}
\hline & $\begin{array}{c}\text { Range for } \\
\text { Emission }\end{array}$ & Diesel Engines & $\begin{array}{c}\text { Caterpiller } \\
\text { Average }\end{array}$ & $\begin{array}{c}\text { Change } \\
\text { GenSet }\end{array}$ \\
\hline $\mathrm{TH}$ & & & & \\
$\mathrm{TH}$ & $0.28-0.37$ & 0.325 & 0.89 & 174 \\
$\mathrm{CO}$ & $0.39-1.9$ & 1.145 & 1.9 & 66 \\
$\mathrm{NO}_{\mathrm{x}}$ & $10.3-14.7$ & 12.5 & 0.7 & -94 \\
$\mathrm{PM}_{10}$ & $0.17-0.26$ & 0.215 & 0.15 & -30 \\
\hline
\end{tabular}

a Data from Rheinhart 1998. 
No data regarding the relative amounts of energy used for these engines were available. However, a duty-cycle-weighted calculation by EF\&EE (1993) indicated an 18\% energy penalty on the basis of the results of tests on a $3516 \mathrm{NG}$ engine and a 3516 diesel engine reported by Caterpillar, Inc. The tests revealed that the energy penalty ranged from about $3 \%$ at full load up to $22 \%$ at $50 \%$ of full load, and increased as the load decreased. For this study, we use the same net energy penalty as EF\&EE (18\%).

\subsubsection{Farming, Commercial/Industrial, and Refrigeration Equipment}

Because no details regarding the population of diesel engines in these source classes were available, we made the following assumptions regarding the sizes of gasoline engines required to replace the diesel engines in these sources. For farm equipment, we assumed that the diesel engines could be replaced by the same gasoline engines used in HD trucks. The corresponding emissions were estimated from HD truck emissions using the following equations:

$$
\mathrm{F}_{\text {Dreplace }}=1.25 \times \mathrm{F}_{\mathrm{D}} \times \mathrm{LHV}_{\mathrm{D}} / \mathrm{LHV}_{\mathrm{G}}
$$

and $\Delta \varepsilon=\mathrm{F}_{\text {Dreplace }} \times\left(\varepsilon_{\mathrm{G}} / \mathrm{F}_{\mathrm{G}}\right)_{\mathrm{HDtrucks}}-\varepsilon_{\mathrm{D}}$

The factor 1.25 in Equation 9 is an estimated de-rating factor to account for the differences in duty cycles to which diesel and gasoline engines are typically subject. For LD commercial and industrial equipment (less than $175 \mathrm{hp}$ ) the following equations were used:

$$
\mathrm{F}_{\text {Dreplace }}=1.1 \times \mathrm{F}_{\mathrm{D}} \times \mathrm{LHV}_{\mathrm{D}} / \mathrm{LHV}_{\mathrm{G}}
$$

and $\Delta \varepsilon=\mathrm{F}_{\text {Dreplace }} \times\left(\varepsilon_{\mathrm{G}} / \mathrm{F}_{\mathrm{G}}\right)_{\text {LDGequip }}-\varepsilon_{\mathrm{D}}$

For HD commercial and industrial equipment (greater than $175 \mathrm{hp}$ ), the following equations were used:

$$
\mathrm{F}_{\text {Dreplace }}=1.25 \times \mathrm{F}_{\mathrm{D}} \times \mathrm{LHV}_{\mathrm{D}} / \mathrm{LHV}_{\mathrm{G}}
$$

and $\Delta \varepsilon=\mathrm{F}_{\text {Dreplace }} \times\left(\varepsilon_{\mathrm{G}} / \mathrm{F}_{\mathrm{G}}\right)_{\mathrm{HDGequip}}-\varepsilon_{\mathrm{D}}$

For refrigeration equipment, all of which was assumed to be fueled by diesel in accordance with CARB (1997b), the following equations were used:

$$
\mathrm{F}_{\text {Dreplace }}=1.1 \times \mathrm{F}_{\mathrm{D}} \times \mathrm{LHV}_{\mathrm{D}} / \mathrm{LHV}_{\mathrm{G}}
$$

and $\Delta \varepsilon=\mathrm{F}_{\text {Dreplace }} \times\left(\varepsilon_{\mathrm{G}} / \mathrm{F}_{\mathrm{G}}\right)_{\text {LDGequip }}-\varepsilon_{\mathrm{D}}$

\subsubsection{Results for Scenario 1}

The changes in fuel use and emissions for this scenario are provided in Tables 12 and 13 for 1995 and 2010, respectively. Note that these results assume that each control scenario was in place long enough for it to be implemented completely (obviously impossible for 1995). 


\subsection{Scenario 2}

\subsubsection{On-Road Vehicles}

\subsubsection{Light-Duty Diesel-Powered Highway Vehicles}

Under Scenario 2, these units would be replaced by catalytically controlled gasoline-fueled vehicles, as in Scenario 1.

\subsubsection{Medium- and Heavy-Duty Trucks and Buses}

MD and HD trucks with diesel engine displacements of less than $8 \mathrm{~L}$ would be replaced by gasoline engines, while those with displacements greater than or equal to $8 \mathrm{~L}$ would be replaced by SI engines burning CNG. For trucks with diesel engine displacements greater than or equal to $8 \mathrm{~L}$ but less than $10 \mathrm{~L}$, the representative CNG engines are the Cummins $10 \mathrm{~L}-240 \mathrm{G}$ and $-260 \mathrm{G}$. For trucks with diesel engine displacements greater than or equal to $10 \mathrm{~L}$, the representative CNG engine is the Caterpillar G3406LE powered by CNG. Data for these and other alternativefueled vehicles are summarized in Table 14 (truck and Orange County Transit Authority [OCTA] bus data from the National Renewable Energy Laboratory [NREL 1996a,b]). Comparison with a control vehicle indicated a 30\% reduction in fuel economy after accounting for the difference in lower heating values. Emissions data from the CBD and COM emission test cycles were used here for the smaller and larger engines, respectively.

Aside from the limited data available, the justification for using different cycles is that the larger engines tend to be used on longer-haul trips (see TIUS data for 1992). The following equations were used to calculate the required replacement fuels:

$$
\mathrm{F}_{\mathrm{G}}=\mathrm{VMT}<_{8 \mathrm{~L}} / \mathrm{VMT}_{\text {Total }} \times \operatorname{Max}\left(\mathrm{FE}_{\mathrm{D}} / \mathrm{FE}_{\mathrm{G}}, \mathrm{LHV}_{\mathrm{D}} / \mathrm{LHV}_{\mathrm{G}} \times \mathrm{Eff}_{\mathrm{D}} / \mathrm{Eff}_{\mathrm{G}}\right) \times \mathrm{F}_{\mathrm{D}}
$$

and $\mathrm{F}_{\mathrm{NG}}=\mathrm{VMT} \geq_{8 \mathrm{~L}} / \mathrm{VMT}_{\text {Total }} \times \mathrm{LHV}_{\mathrm{D}} / \mathrm{LHV}_{\mathrm{NG}} \times(1+0.30) \times \mathrm{F}_{\mathrm{D}}$

The factor 0.30 in Equation 18 represents the loss of engine efficiency obtained in the 14-month test reported in NREL 1996a. Note that the VMT factors in Equations 17, 18, and 19 refer to specific truck size classes, e.g. $\mathrm{VMT}_{\text {Total }}$ refers to the total VMT within a given size class. The change in emissions was computed by using Equations 19 and 20 for MD and HD trucks, respectively.

For MD trucks:

$$
\begin{gathered}
\Delta \varepsilon_{\mathrm{MD}}=\left[\left(\varepsilon_{\mathrm{G}} / \mathrm{F}_{\mathrm{G}}\right)_{\mathrm{MDtrucks}} \times \operatorname{Max}\left(\mathrm{FE}_{\mathrm{D}} / \mathrm{FE}_{\mathrm{G}}, \mathrm{LHV}_{\mathrm{D}} / \mathrm{LHV}_{\mathrm{G}} \times \mathrm{Eff}_{\mathrm{D}} / \mathrm{Eff}_{\mathrm{G}}\right) \times \mathrm{F}_{\mathrm{D}}-\varepsilon_{\mathrm{D}}\right] \times \\
\mathrm{VMT}_{\mathrm{MD}<8 \mathrm{~L}} / \mathrm{VMT}_{\mathrm{MD} \text { Total }}+\left[\left(\mathrm{EF}_{\mathrm{NG}} / \mathrm{EF}_{\mathrm{D}}\right)_{10 \mathrm{~L}}-1\right] \times \varepsilon_{\mathrm{D}} \times \mathrm{VMT}_{\mathrm{MD}} \geq_{8 \mathrm{~L}} / \mathrm{VMT}_{\mathrm{MD} \text { Total }}
\end{gathered}
$$

EF stands for emission factor (averaged over field tests in four cities) and the subscript "10L" refers to the diesel and NG versions of the Cummins 10-L engine. 
Table 12 Estimated Increases in Energy and Emissions Under Scenario 1, 1995

\begin{tabular}{|c|c|c|c|c|c|c|c|c|c|c|c|c|}
\hline \multirow[b]{2}{*}{ Source Category } & \multirow[b]{2}{*}{$\begin{array}{c}\text { Added } \\
\text { Daily } \\
\text { Energy } \\
\left(10^{6} \mathrm{Btu}\right)\end{array}$} & \multirow[b]{2}{*}{$\begin{array}{c}\text { Added } \\
\text { Fuel/Day } \\
\left(10^{3} \mathrm{gal}\right) \\
\text { Gasoline } \\
\end{array}$} & \multirow[b]{2}{*}{$\begin{array}{c}\text { Added } \\
\text { Fuel/Day } \\
\left(10^{3} \mathrm{gal}\right) \\
\text { LNG } \\
\end{array}$} & \multicolumn{9}{|c|}{ Daily Emissions (tons/d) from Combustion } \\
\hline & & & & ROG & co & $\mathrm{NO}_{\mathrm{x}}$ & $\begin{array}{c}\mathrm{CO}_{2} \\
\left(10^{3}\right)\end{array}$ & $\mathbf{P M}_{10}$ & $\mathrm{SO}_{\mathrm{x}}$ & $\mathrm{N}_{2} \mathrm{O}$ & $\mathrm{CH}_{4}$ & $\begin{array}{c}\text { GHGs } \\
\left(\mathrm{CO}_{2^{-}}\right. \\
\text {equivalent }) \\
\left(10^{3}\right) \\
\end{array}$ \\
\hline \multicolumn{13}{|l|}{ On-Road Mobile Sources } \\
\hline Autos (gasoline) & 2,016 & 175 & 0 & 4.93 & 61.25 & -0.18 & -0.03 & -1.40 & 0.00 & 1.19 & 0.15 & 0.346 \\
\hline LD Trucks (gasoline) & 1,254 & 91 & 0 & 2.58 & 34.45 & 1.53 & 0.00 & -0.79 & 0.01 & 0.58 & 0.08 & 0.181 \\
\hline MD Trucks (gasoline) & 52,566 & 1,661 & 0 & 21.22 & 192.32 & 24.48 & 3.35 & -3.21 & 0.18 & 5.29 & 0.42 & 4.998 \\
\hline HD Trucks (gasoline) & 532,807 & 10,336 & 0 & 13.95 & 682.53 & 107.05 & 40.24 & -33.35 & 7.07 & 49.94 & 3.74 & 55.799 \\
\hline Buses (gasoline) & 8,849 & 244 & 0 & 0.69 & 31.30 & 1.29 & 1.74 & -0.14 & 0.28 & 2.17 & 0.20 & 2.416 \\
\hline Subtotal & 597,493 & 12,507 & 0 & 43.37 & 1001.84 & 134.17 & 45.30 & -38.88 & 7.54 & 59.18 & 4.60 & 63.741 \\
\hline \multicolumn{13}{|l|}{ Off-Road Mobile Sources } \\
\hline Trains (locomotives) - NG SI engines & 13,852 & 0 & 1,246 & 12.18 & 15.18 & -141.00 & -0.89 & -0.90 & -16.51 & 0.00 & 0.87 & -0.873 \\
\hline Vessels - NG SI engines & 10,379 & 0 & 933 & 7.15 & 3.47 & -39.56 & -0.67 & -0.89 & -12.37 & 0.00 & 0.65 & -0.654 \\
\hline Farm equipment (gasoline) & 14,554 & 631 & 0 & -4.90 & 28.25 & -60.64 & 0.92 & -5.64 & -10.94 & 0.99 & 0.04 & 1.228 \\
\hline LD non-farm equipment (gasoline) & 1,431 & 136 & 0 & 10.10 & 357.51 & -16.30 & -0.54 & -1.39 & -2.73 & 0.33 & 0.02 & -0.436 \\
\hline HD non-farm equipment (gasoline) & 23,245 & 1,007 & 0 & 0.63 & 215.16 & -95.01 & 1.16 & -4.60 & -17.73 & 2.22 & 0.10 & 1.847 \\
\hline Refrigeration units (gasoline) & 691 & 66 & 0 & 4.61 & 171.49 & -8.14 & -0.26 & -0.81 & -1.31 & 0.53 & 0.08 & -0.091 \\
\hline Subtotal & 64,153 & 1,840 & 2,179 & 29.78 & 791.06 & -360.64 & -0.28 & -14.22 & -61.59 & 4.06 & 1.76 & 1.020 \\
\hline Stationary Sources (natural gas) & 3,110 & 0 & 280 & 2.96 & 8.53 & -38.82 & -0.20 & -1.15 & -3.71 & 0.00 & 0.00 & -0.200 \\
\hline Total - All Sources & 664,757 & 14,347 & 2,459 & 76.10 & 1801.43 & -265.29 & 44.82 & -54.25 & -57.76 & 63.24 & 6.36 & 64.561 \\
\hline
\end{tabular}


Table 13 Estimated Increases in Energy and Emissions Under Scenario 1, 2010

\begin{tabular}{|c|c|c|c|c|c|c|c|c|c|c|c|c|}
\hline \multirow[b]{2}{*}{ Source Category } & \multirow[b]{2}{*}{$\begin{array}{c}\text { Added } \\
\text { Daily } \\
\text { Energy } \\
\left(10^{6} \mathrm{Btu}\right) \\
\end{array}$} & \multirow[b]{2}{*}{$\begin{array}{l}\text { Added } \\
\text { Fuel/Day } \\
\left(10^{3} \text { gal) }\right. \\
\text { Gasoline } \\
\end{array}$} & \multirow[b]{2}{*}{$\begin{array}{c}\text { Added } \\
\text { Fuel/Day } \\
\left(10^{3} \text { gal) }\right. \\
\text { LNG } \\
\end{array}$} & \multicolumn{9}{|c|}{ Daily Emissions from Combustion (tons/d) } \\
\hline & & & & ROG & co & $\mathrm{NO}_{\mathrm{x}}$ & $\begin{array}{l}\mathrm{CO}_{2} \\
\left(10^{3}\right) \\
\end{array}$ & $\mathrm{PM}_{10}$ & sox & $\mathrm{N}_{2} \mathrm{O}$ & $\mathrm{CH}_{4}$ & $\begin{array}{c}\text { GHGs } \\
\left(\mathrm{CO}_{2} \text {-equivalent }\right) \\
\left(10^{3}\right) \\
\end{array}$ \\
\hline \multicolumn{13}{|l|}{ On-Road Mobile Sources } \\
\hline Autos (gasoline) & 202 & 18 & 0 & -0.04 & 0.50 & -0.55 & 0.01 & -0.15 & 0.00 & 0.02 & 0.01 & 0.02 \\
\hline LD Trucks (gasoline) & 47 & 4 & 0 & -0.02 & 0.09 & -0.09 & 0.00 & -0.04 & 0.00 & 0.01 & 0.00 & 0.00 \\
\hline MD Trucks (gasoline) & 83,201 & 2,629 & 0 & 10.16 & 133.26 & 6.65 & 5.63 & -1.60 & 0.08 & 7.69 & 0.80 & 8.03 \\
\hline HD Trucks (gasoline) & 637,436 & 12,366 & 0 & 15.70 & 908.42 & 7.78 & 47.88 & -8.47 & 6.58 & 68.63 & 5.86 & 69.28 \\
\hline Buses (gasoline) & 13,083 & 281 & 0 & 0.84 & 36.45 & 6.41 & 2.26 & 0.11 & 0.38 & 2.51 & 0.25 & 3.04 \\
\hline Subtotal & 733,970 & 15,298 & 0 & 26.64 & 1078.73 & 20.20 & 55.78 & -10.14 & 7.04 & 78.86 & 6.91 & 80.37 \\
\hline \multicolumn{13}{|l|}{ Off-Road Mobile Sources } \\
\hline Trains (locomotives) - NG SI engines & 18,338 & 0 & 1,649 & 12.18 & 13.86 & -131.60 & -1.18 & -0.90 & -21.86 & 0.00 & 1.15 & -1.16 \\
\hline Vessels - NG SI engines & 16,004 & 0 & 1,439 & 14.36 & 5.18 & -30.43 & -1.03 & -1.02 & -19.08 & 0.00 & 1.01 & -1.01 \\
\hline Farm equipment (gasoline) & 18,251 & 791 & 0 & -8.02 & 43.06 & -93.66 & 1.15 & -7.06 & -13.72 & 1.25 & 0.09 & 1.53 \\
\hline LD non-farm equipment (gasoline) & 1,676 & 160 & 0 & 11.82 & 418.64 & -19.08 & -0.63 & -1.63 & -3.19 & 0.56 & 0.03 & -0.46 \\
\hline HD non-farm equipment (gasoline) & 28,848 & 1,250 & 0 & 0.78 & 267.01 & -117.91 & 1.44 & -5.71 & -22.01 & 1.97 & 0.15 & 2.05 \\
\hline Refrigeration units (gasoline) & 867 & 83 & 0 & 5.78 & 215.04 & -10.21 & -0.32 & -1.01 & -1.64 & 0.13 & 0.05 & -0.28 \\
\hline Subtotal & 83,983 & 2,283 & 3,088 & 36.92 & 962.79 & -402.89 & -0.58 & -17.32 & -81.49 & 3.91 & 2.48 & 0.69 \\
\hline $\begin{array}{l}\text { Stationary Sources } \\
\text { (natural gas) }\end{array}$ & 3,384 & 0 & 304 & 3.95 & 9.00 & -34.34 & -0.12 & -1.22 & -3.81 & 0.00 & 0.00 & -0.12 \\
\hline Total - All Sources & 821,336 & 17,581 & 3,392 & 67.51 & 2050.53 & -417.02 & 55.08 & -28.68 & -78.27 & 82.77 & 9.40 & 80.93 \\
\hline
\end{tabular}


Table 14 Fuel Use and Emission Rates for Heavy-Duty Vehicles Powered by Alternative Fuelsa

\begin{tabular}{|c|c|c|c|c|c|c|c|c|c|c|c|}
\hline \multirow[b]{2}{*}{ Vehicle } & \multirow[b]{2}{*}{$\begin{array}{c}\text { Fuel/Cycle } \\
\text { (no. of tests) }\end{array}$} & \multirow[b]{2}{*}{$\begin{array}{c}\text { Fuel Use } \\
\text { (mi/gal) }\end{array}$} & \multicolumn{9}{|c|}{ Emission Rates ( $\mathrm{g} / \mathrm{mi})$} \\
\hline & & & ROG & $\mathrm{co}$ & $\mathrm{NO}_{\mathrm{x}}$ & $\mathrm{CO}_{2}$ & $\mathrm{PM}_{10}$ & $\mathrm{So}_{\mathrm{x}}$ & THC & $\begin{array}{c}\text { Formaldehyde } \\
(\mathrm{HCHO})\end{array}$ & $\mathrm{CH}_{4}$ \\
\hline \multirow[t]{3}{*}{ Cat. G3406LE tractor $(14.6 \mathrm{~L})^{\mathrm{b}}$} & CNG/CBD (1) & 1.18 & 5.747 & 0.25 & 31.10 & 4465 & 0.037 & 0.13 & 114.90 & NM & 109.38 \\
\hline & CNG/EPA (4) & 2.27 & 2.015 & 0.23 & 19.55 & 2370 & 0.060 & 0.07 & 40.28 & 0.01 & 38.34 \\
\hline & $\mathrm{CNG} / \mathrm{COM}(3)$ & 3.18 & 0.787 & 0.10 & 16.83 & 1722 & 0.014 & 0.05 & 15.73 & 0.01 & 14.98 \\
\hline \multirow[t]{2}{*}{ DDC 6V-92TA sludge hauler } & Meth/CBD & 2.27 & $N M^{c}$ & 66.38 & 13.47 & 3964 & 1.241 & 0.00 & 8.25 & 1.65 & NM \\
\hline & Meth/EPA & 4.42 & NM & 19.04 & 8.62 & 2064 & 0.425 & 0.00 & 3.14 & 1.06 & NM \\
\hline Cat. 3306B Ref. Hauler & Meth/CBD & 3.74 & NM & 20.80 & 28.30 & 2430 & 0.120 & 0.00 & 8.10 & 0.95 & NM \\
\hline Cummins L10 240G OCTA bus & $\mathrm{CNG} / \mathrm{CBD}$ & 2.32 & 0.206 & 0.00 & 6.05 & 2981 & 0.043 & 0.07 & 4.12 & 0.03 & 3.92 \\
\hline Cummins L10 OCTA bus & LPG/CBD & 2.54 & NM & 0.02 & 3.04 & 3618 & 0.036 & 0.07 & 3.09 & 0.03 & NM \\
\hline Cummins L10 OCTA bus & LPG/COM & 5.24 & NM & 0 & 0.22 & 1758 & 0.014 & 0.04 & 0.11 & NM & NM \\
\hline Cummins L10 OCTA bus & $\mathrm{D} / \mathrm{CBD}$ & 4.22 & NM & 14.83 & 31.08 & 2421 & 0.538 & 0.66 & 1.11 & 0.07 & 0.02 \\
\hline Cummins L10-240G Miami bus & $\mathrm{CNG/CBD}$ & 3.40 & 1.030 & 15.80 & 29.00 & 1957 & 0.010 & 0.05 & 20.60 & NM & 19.61 \\
\hline Cummins L10-240G Tacoma bus & $\mathrm{CNG} / \mathrm{CBD}$ & 4.50 & 0.465 & 21.80 & 30.40 & 1481 & 0.010 & 0.03 & 9.30 & NM & 8.85 \\
\hline Cummins L10-260G NY bus & $\mathrm{CNG} / \mathrm{CBD}$ & 2.60 & 0.805 & 1.60 & 12.00 & 2621 & 0.030 & 0.06 & 16.10 & NM & 15.33 \\
\hline Cummins L10-260G Tacoma bus & $\mathrm{CNG} / \mathrm{CBD}$ & 4.50 & 0.775 & 0.70 & 11.20 & 1496 & 0.020 & 0.03 & 15.50 & NM & 14.76 \\
\hline Cummins L10 Diesel Miami bus & $\mathrm{D} / \mathrm{CBD}$ & 3.50 & 1.985 & 23.50 & 22.00 & 2903 & 1.990 & 0.71 & 1.90 & NM & 0.03 \\
\hline Cummins L10 Diesel Tacoma bus & $\mathrm{D} / \mathrm{CBD}$ & 5.80 & 2.508 & 11.20 & 24.60 & 1751 & 1.740 & 0.40 & 2.40 & NM & 0.04 \\
\hline Cummins L10 Diesel NY bus & $D / C B D$ & 3.00 & & & & & & & & & 0.00 \\
\hline
\end{tabular}

a Data from "Alternative Fuel Transit Buses." Bus length $=40 \mathrm{ft}$.

b This engine was installed in a Ford LTLA-9000 AeroMax tractor operated by Vons Companies, Inc., and tested for 14 months, starting on Oct. 12 , 1992. See "Alternative-Fueled Truck Demonstration Natural Gas Program: Caterpillar G3406LE Development and Demonstration." This report is available from NTIS at the following web address http://www.afdc.nrel.gov/demopro//hdv/hdvrpts/vonsec1.html

c $\mathrm{NM}=$ not measured 
For HD trucks:

$$
\begin{aligned}
& \Delta \varepsilon_{\mathrm{HD}}=\left(\varepsilon_{\mathrm{G}} / \mathrm{F}_{\mathrm{G}}\right)_{\mathrm{HD} \text { trucks }} \times \operatorname{Max}\left(\mathrm{FE}_{\mathrm{D}} / \mathrm{FE}_{\mathrm{G}}, \mathrm{LHV}_{\mathrm{D}} / \mathrm{LHV}_{\mathrm{G}} \times \mathrm{Eff}_{\mathrm{D}} / \mathrm{Eff}_{\mathrm{G}}\right) \times\left(\mathrm{F}_{\mathrm{D}}-\varepsilon_{\mathrm{D}}\right) \times \\
& \mathrm{VMT}_{\mathrm{HD}<8 \mathrm{~L}} / \mathrm{VMT}_{\mathrm{HD} \text { Total }}+\left[\mathrm{A} \times\left(\mathrm{EF}_{\mathrm{NG}} / \mathrm{EF}_{\mathrm{D}}\right)_{14 \mathrm{~L}}+(1-\mathrm{A}) \times\left(\mathrm{EF}_{\mathrm{NG}} / \mathrm{EF}_{\mathrm{D}}\right)_{10 \mathrm{~L}}-1\right] \times \varepsilon_{\mathrm{D}} \times \\
& \mathrm{VMT}_{\mathrm{HD}} \geq 8 \mathrm{~L} / \mathrm{VMT}_{\mathrm{HD} \text { Total }}
\end{aligned}
$$

where $\quad \mathrm{A}=\mathrm{VMT}_{>10 \mathrm{~L}} / \mathrm{VMT}_{\text {Total }}$

Note that Equations 19 and 20 differ both in terms of MD vs. HD parameters and in the factor "A" in Equation 20. Factor "A" is the ratio of the VMT for HD trucks with displacements greater than $10 \mathrm{~L}$ to the VMT for all HD trucks. These trucks would be replaced by trucks with the representative 14.5-L Caterpillar G3406LE powered by a CNG engine. The remainder of HD diesels (1-A) having displacements equal to or greater than $8 \mathrm{~L}$ would be replaced by Cummins 10 -L engines fueled by CNG. One additional complication in Equation 20 is that no data are available regarding a diesel control for the 14.5-L Caterpillar G3406LE CNG engine. Consequently, we had to substitute a nearly equivalent diesel control engine, the Cummins 855 diesel engine that was tested on the University of West Virginia (UWV) transient test cycle.

Diesel buses measuring $27.5 \mathrm{ft}$ long or less would be replaced with gasoline-fueled units as in Scenario 1. Buses greater than $27.5 \mathrm{ft}$ long (all assumed to be diesel-fueled) would be replaced with SI engines burning CNG. The representative SI replacement engine is the Cummins L10$240 \mathrm{G}$ (see test data in Table 14). Emissions from the CBD emissions test cycle were employed in this case. The fuel use and emissions changes were computed from Equations 21 and 22.

$$
\mathrm{F}_{\mathrm{G}}=\text { fbuses }_{<27.5 \mathrm{ft}} \times \mathrm{VMT}_{\text {Dbuses }} / \mathrm{FE}_{10-16 \mathrm{k} \mathrm{lb} \mathrm{G} \mathrm{trucks}}
$$

$\mathrm{F}_{\mathrm{NG}}=$ fbuses $_{>27.5 \mathrm{ft}} \times \mathrm{VMT}_{\text {Dbuses }} / \mathrm{Min}\left(\mathrm{FE}_{\mathrm{NG} \text { buses }}, \mathrm{LHV}_{\mathrm{NG}} / \mathrm{LHV}_{\mathrm{D}} \times \mathrm{Eff}_{\mathrm{NG}} / \mathrm{Eff}_{\mathrm{D}} \times \mathrm{FE}_{\mathrm{D} \text { buses }}\right)$

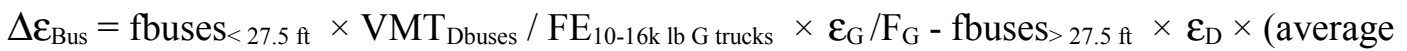
emission factor for NG buses)/(average emission factor for diesel buses) $-\varepsilon_{\mathrm{D}}$

The "average emission factors" for NG and diesel buses are averages for field tests in three areas: Orange County, California; Miami, Florida; and Tacoma, Washington. The term " $\varepsilon_{\mathrm{D}}$ " is the base case emissions for diesel buses, and the terms " $\varepsilon_{\mathrm{G}}$ " and " $F_{\mathrm{G}}$ " refer to the emissions and fuel use of MD catalytically controlled gasoline-fueled trucks. (No data on field tests of gasolinefueled buses were found).

\subsubsection{Off-Road Sources}

\subsubsection{Locomotives and Vessels}

Under Scenario 2, locomotives and vessels would be dual-fueled (LNG and pilot diesel) and would operate on the California duty cycle. Our representative engines are the EMD 16 645E3B (unmodified) and the same engine modified to use dual fuel with a conversion kit supplied by Energy Conversions, Inc. (Jensen 1998). The emission factors for the unmodified engine are shown in Tables 9a and 9b. The emission factors for the dual-fuel engine are listed in Table 15. 
Table 15 Duty-Cycle-Weighted Emission Factors for the Dual-Fuel EMD 16-645 Locomotive Engine

\begin{tabular}{|c|c|c|c|c|c|c|c|c|c|}
\hline \multirow{2}{*}{$\begin{array}{l}\text { Throttle } \\
\text { Position }\end{array}$} & \multirow{2}{*}{$\begin{array}{c}\% \text { Time } \\
\text { in Notch }\end{array}$} & \multirow{2}{*}{ Horsepower } & \multicolumn{7}{|c|}{ Emission Rates (g/bhp-h) } \\
\hline & & & ROG & $\mathrm{CO}$ & $\mathrm{NO}_{x}$ & $\mathrm{CO}_{2}$ & PM & $\mathrm{SO}_{\mathrm{x}}$ & NMHC \\
\hline Off & 23.00 & 0.00 & 0.00 & 0.00 & 0.00 & 0 & 0.00 & 0.00 & 0.00 \\
\hline Brake & 6.10 & 138.00 & 47.26 & 74.92 & 332.51 & 12501 & 13.97 & 44.53 & 46.30 \\
\hline Idle & 39.70 & 241.00 & 243.20 & 607.55 & 3453.94 & 53196 & 180.10 & 301.38 & 238.24 \\
\hline 1 & 3.00 & 312.00 & 23.89 & 53.82 & 149.76 & 6426 & 10.90 & 20.08 & 23.40 \\
\hline 2 & 3.20 & 584.00 & 27.66 & 55.13 & 238.27 & 8129 & 12.80 & 28.03 & 27.10 \\
\hline 3 & 3.10 & 857.00 & 117.97 & 389.21 & 151.43 & 10693 & 6.64 & 10.23 & 115.57 \\
\hline 4 & 3.90 & 1213.00 & 48.29 & 391.07 & 312.23 & 17172 & 6.78 & 14.03 & 47.31 \\
\hline 5 & 3.10 & 1681.00 & 42.56 & 416.89 & 276.19 & 18656 & 7.82 & 12.51 & 41.69 \\
\hline 6 & 2.90 & 2247.00 & 28.83 & 417.04 & 286.72 & 21960 & 9.34 & 12.82 & 28.24 \\
\hline 7 & 2.20 & 3002.00 & 26.97 & 518.45 & 270.78 & 23380 & 11.56 & 8.59 & 26.42 \\
\hline 8 & 9.90 & 3359.00 & 203.68 & 3059.38 & 1152.81 & 113396 & 62.07 & 44.34 & 199.52 \\
\hline $\begin{array}{l}\text { Weighted } \\
\text { Averages }\end{array}$ & & & 1.12 & 8.29 & 9.18 & 396 & 0.45 & 0.69 & 1.10 \\
\hline
\end{tabular}

These engines were field-tested by Burlington Northern Railroad in freight service (Olsen 1998). Fuel use for both locomotives and vessels was computed as follows.

$$
\mathrm{F}_{\mathrm{PD}}=\mathrm{Eff}_{\mathrm{D}} / \mathrm{Eff}_{\mathrm{NG}} \times \% \mathrm{PD} / 100 \% \times \mathrm{F}_{\mathrm{D}}
$$

and $\mathrm{F}_{\mathrm{NG}}=\mathrm{Eff}_{\mathrm{D}} / \mathrm{Eff}_{\mathrm{NG}} \times \% \mathrm{NG} / 100 \% \times \mathrm{LHV}_{\mathrm{D}} / \mathrm{LHV}_{\mathrm{NG}} \times \mathrm{F}_{\mathrm{D}}$

We assumed for these equations that pilot diesel was used $6 \%$ of the time and that the ratio of efficiencies was 1.0459 (average of 1.032 and 1.06) (Olsen 1997). The change in emissions from both locomotives and vessels was estimated by taking a simple ratio of duty-cycle-weighted emission factors multiplied by the base-case emissions:

$$
\Delta \varepsilon=(\text { emission factor for DF }) /(\text { emission factor for D }) \times \varepsilon_{\mathrm{D}}
$$

\subsubsection{Mobile Equipment}

Units in this category that use diesel fuel in CI engines would be replaced by units equipped with gasoline-fueled engines. The estimation procedures used here were identical to those described in Section 3.2.2.2. 


\subsubsection{Stationary Diesel Engines}

Because no details were available for these engine populations, we assumed that stationary diesel engines could be replaced by dual-fuel engines operating at full load (see Table 16 for the full-load emission factors used). The same representative engines listed in Section 3.3.2.1 were assumed here.

\subsubsection{Results for Scenario 2}

The results for this scenario are presented in Table 17. The amount of energy used in combustion would increase for all source categories under Scenario 2. There would be a net increase in the emissions of ROG, $\mathrm{CO}, \mathrm{N}_{2} \mathrm{O}$, and $\mathrm{CH}_{4}$, and $\mathrm{GHGs}$ (expressed in terms of $\mathrm{CO}_{2}-$ equivalent emissions, even though the actual emissions of $\mathrm{CO}_{2}$ decrease). Comparing the results for Scenarios 1 and 2 (Tables 12 and 17, respectively) reveals that the added energy for Scenario 2 is quite a bit lower than that for Scenario 1.

Two points should be noted. First, the total energy for the base case for 2010 is $1.45 \times 10^{12} \mathrm{Btu} /$ day; the added energies estimated for Scenarios 1 and 2 are, respectively, $0.821 \times 10^{12}$ and $0.286 \times 10^{12} \mathrm{Btu} / \mathrm{day}$. That is, the changes are the same order of magnitude as the base case, and are therefore significant. Second, the main contribution to the difference between the added energies for the two scenarios is from HD trucks. For Scenario 1, each HD diesel-fueled truck was replaced by two gasoline-fueled trucks with smaller engines (less than 8 L). For Scenario 2, each HD diesel-fueled truck was replaced by one truck powered by a HD SI engine burning NG. The HD SI engines are built from the same blocks as their CI diesel engine counterparts, and have similar ruggedness, rpm, and torque characteristics. Field tests have shown that they provide similar performance, although they must be de-rated for differences in the energy content of the fuels, differences in engine efficiency resulting from different compression ratios, and other factors (including effectiveness of turbochargers). As noted above, de-ratings can range in horsepower from $10 \%$ to $50 \%$ depending on the engine type and components used to enhance the diesel engine performance.

\subsection{Scenario 3}

There are three replacement fuels dispensed as liquids that can be relatively easily used in conventional CI engines: biodiesel, FTD, and DME. All three offer some emissions benefits. Both FTD and DME can be manufactured from NG and are therefore not limited by feedstock availability. Biodiesel, on the other hand, is produced from vegetable (and some waste animal) oils; the supply of these oils for non-nutritional uses is presently very limited. Of the three, FTD is most compatible with existing infrastructure for conventional diesel. Only minimal adjustments would be required to obtain optimal performance from existing CI engines fueled by FTD. Its physical properties are very similar to those of number 2 diesel (\#2D) fuel, and its chemical properties are superior because the FT process yields middle distillates very low in aromatics and sulfur compounds; the substitute straight-chain hydrocarbons (predominantly paraffins) provide a better cetane rating than \#2D. Use of DME would require modification of diesel injector pressures because it must be stored on-board as a lightly pressurized liquid. Only 
Table 16 Large Diesel and Dual-Fuel Engine Emission Factors at Full Load

\begin{tabular}{|c|c|c|c|c|c|c|c|c|c|}
\hline \multirow[b]{2}{*}{ Reciprocating Engines } & \multicolumn{9}{|c|}{ Emission Rates (g/bh/h) } \\
\hline & ROG & $\mathrm{CO}$ & $\mathrm{NO}_{\mathrm{X}}$ & $\mathrm{CO}_{2}$ & $\mathbf{P M}_{10}$ & so $_{x}$ & THC & NMHC & $\mathrm{CH}_{4}$ \\
\hline $\begin{array}{l}\text { Burlington Northern unmodified EMD } 16-645 \text { on diesel (fuel flow } \\
@ \text { full load }=713 \mathrm{gal} / \mathrm{h} \text { ) }\end{array}$ & 0.21 & 0.50 & 10.90 & 397 & 0.25 & 1.39 & 0.20 & 0.20 & 0.00 \\
\hline Burlington Northern modified EMD $16-645$ on diesel & 0.32 & 0.20 & 7.90 & 396 & 0.36 & 1.39 & 0.30 & 0.30 & 0.00 \\
\hline Burlington Northern modified EMD $16-645$ on diesel plus NGa & 0.40 & 8.60 & 3.60 & 316 & 0.19 & 0.13 & 7.90 & 0.90 & 7.00 \\
\hline MUSE 1,500-KW DF Gen Set, before conversion, $2,119 \mathrm{hp}$ & $\mathrm{NM}^{\mathrm{b}}$ & 0.25 & 10.50 & NM & 0.35 & NM & NM & NM & NM \\
\hline MUSE $1,500-K W$ DF Gen Set, after conversion, $2,119 \mathrm{hp}$ & NM & 11.40 & 2.40 & NM & 0.21 & NM & NM & NM & NM \\
\hline Caterpillar 3516 Gen Set, 1,200 rpm, 1,034 hp, NG, full load & 0.61 & 1.50 & 2.00 & NM & & NM & 3.90 & 0.60 & 3.30 \\
\hline $\begin{array}{l}\text { Caterpillar } 3616 \text { Gen Set, } 900 \text { rpm, } 4,021 \text { hp, NG, full load } \\
\text { Gas Turbines }\end{array}$ & 0.91 & 1.90 & 0.70 & NM & 0.15 & NM & 5.95 & 0.89 & 5.06 \\
\hline Eurodyn gas turbine, NG, 3,472 hp (@236.2 gal//hc) & 0.04 & 0.96 & 0.84 & 0.84 & NM & 0.00 & NM & 0.72 & NM \\
\hline Eurodyn gas turbine, liquid fuel, $3,472 \mathrm{hp}\left(@ 237.8 \mathrm{gal} / \mathrm{h}^{\mathrm{c}}\right.$ ) & 0.75 & 0.96 & 1.08 & 0.84 & NM & 1.26 & NM & 0.72 & NM \\
\hline$@$ full load, energy consumption $=10.8 \mathrm{MJ} / \mathrm{kWh}=28 \mathrm{GJ} / \mathrm{h}$ & NM & NM & NM & NM & NM & NM & NM & NM & NM \\
\hline$@ 3 / 4 \mathrm{load}$, energy consumption $=11.9 \mathrm{MJ} / \mathrm{kWh}=23 \mathrm{GJ} / \mathrm{h}$ & NM & NM & NM & NM & NM & NM & NM & NM & NM \\
\hline @ $1 / 2$ load, energy consumption $=14.4 \mathrm{MJ} / \mathrm{kWh}=18.6 \mathrm{GJ} / \mathrm{h}$ & NM & NM & NM & NM & NM & NM & NM & NM & NM \\
\hline Makila TM1600 gas turbine on diesel (duty-cycle weighted) & 0.28 & 1.73 & 2.06 & 949.95 & 0.19 & 1.83 & 0.27 & NM & NM \\
\hline Makila TM1600 gas turbine on diesel (full load) & 0.16 & 0.53 & 2.83 & 0.69 & 0.04 & 1.33 & 0.16 & NM & NM \\
\hline
\end{tabular}

Note: Trains using Makila gas turbines require two engines: a diesel engine and a hoteling engine. Turbo trains are used only in passenger service.

a Fuel consumption was greater by $3.2-6.7 \%$ during train runs (average $=4.95 \%$ greater).

$\mathrm{NM}=$ Not measured.

c Diesel-equivalent gal/h. 
Table 17 Estimated Increases in Energy and Emissions Under Scenario 2, 2010

\begin{tabular}{|c|c|c|c|c|c|c|c|c|c|c|c|c|}
\hline \multirow[b]{2}{*}{ Source Category } & \multirow[b]{2}{*}{$\begin{array}{c}\text { Added Daily } \\
\text { Energy } \\
\left(10^{6} \text { Btu }\right)\end{array}$} & \multirow[b]{2}{*}{$\begin{array}{l}\text { Added } \\
\text { Fuel/Day } \\
\left(10^{3} \text { gal }\right)\end{array}$} & \multirow[b]{2}{*}{$\begin{array}{c}\text { Added } \\
\text { Fuel/Day } \\
\left(10^{3} \mathrm{gal}\right)\end{array}$} & \multicolumn{9}{|c|}{ Increased Daily Emissions (tons/day) Due to Operation } \\
\hline & & & & ROG & Co & $\mathrm{NO}_{\mathrm{x}}$ & $\mathrm{CO}_{2}\left(10^{3}\right)$ & $\mathbf{P M}_{10}$ & $\mathrm{SO}_{\mathrm{x}}$ & $\mathrm{N}_{2} \mathrm{O}$ & $\mathrm{CH}_{4}$ & $\begin{array}{c}\text { GHGs } \\
\left(\mathrm{CO}_{2} \text {-equivalent }\right) \\
\left(10^{3}\right)\end{array}$ \\
\hline On-Road Mobile Sources & & Gasoline & CNG & & & & & & & & & \\
\hline Autos & 202 & 18 & 0 & -0.04 & 0.50 & -0.55 & 0.01 & -0.15 & 0.00 & 0.02 & 0.01 & 0.02 \\
\hline LD trucks & 47 & 4 & 0 & -0.02 & 0.09 & -0.09 & 0.00 & -0.04 & 0.00 & 0.01 & 0.00 & 0.00 \\
\hline MD trucks & 34,452 & 1,611 & 943 & 5.77 & 69.11 & -4.59 & -2.35 & -2.08 & -1.88 & 4.68 & 7.74 & -0.73 \\
\hline HD trucks & 181,180 & 2,839 & 8,822 & -16.77 & -32.37 & -44.16 & -8.21 & -12.97 & -11.22 & 15.64 & 111.05 & -1.03 \\
\hline Buses & 10,546 & 17 & 384 & -1.32 & 0.84 & -5.21 & -0.95 & -0.08 & -0.40 & 0.17 & 3.59 & -0.82 \\
\hline Subtotal & 226,428 & 4,488 & 10,149 & -12.38 & 38.17 & -54.61 & -11.49 & -15.31 & -13.51 & 20.52 & 122.39 & -2.56 \\
\hline \multicolumn{13}{|l|}{ Off-Road Mobile Sources } \\
\hline Trains (locomotives) & 5,043 & 50 & 1,379 & 5.50 & 100.09 & -53.71 & -0.63 & -0.26 & -13.11 & -0.01 & 34.85 & 0.10 \\
\hline \multirow[t]{2}{*}{ Vessels } & 4,401 & 44 & 1,203 & 6.49 & 37.38 & -12.42 & -0.55 & -0.29 & -25.54 & -0.01 & 30.41 & 0.09 \\
\hline & & Gasoline & & & & & & & & & & \\
\hline Farm equipment (diesel) & 18,251 & 791 & 0 & -8.02 & 43.06 & -93.66 & 1.15 & -7.06 & -13.72 & 1.25 & 0.09 & 1.53 \\
\hline $\begin{array}{l}\text { LD non-farm equipment } \\
\text { (diesel) }\end{array}$ & 1,676 & 160 & 0 & 11.82 & 418.64 & -19.08 & -0.63 & -1.63 & -3.19 & 0.56 & 0.03 & -0.46 \\
\hline $\begin{array}{l}\text { HD non-farm equipment } \\
\text { (diesel) }\end{array}$ & 28,848 & 1,250 & 0 & 0.78 & 267.01 & -117.91 & 1.44 & -5.71 & -22.01 & 1.97 & 0.15 & 2.05 \\
\hline Refrigeration units (diesel) & 867 & 83 & 0 & 5.78 & 215.04 & -10.21 & -0.32 & -1.01 & -1.64 & 0.13 & 0.05 & -0.28 \\
\hline Subtotal & 59,085 & 2,376 & 2,582 & 22.37 & $1,081.22$ & -306.99 & 0.45 & -15.95 & -79.21 & 3.90 & 65.58 & 3.04 \\
\hline Stationary Sources & 930 & 9 & 254 & 10.22 & 234.64 & 13.19 & 1.29 & 3.09 & 0.36 & 0.00 & 0.00 & 1.29 \\
\hline Total - All Sources & 286,444 & 6,874 & 12,985 & 20.20 & $1,354.03$ & -348.41 & -9.75 & -28.18 & -92.36 & 24.42 & 187.97 & 1.77 \\
\hline
\end{tabular}


FTD and DME are considered as feasible near-term substitutes in our analysis. For Scenario 3, FTD would replace diesel.

Southwest Research Institute (Leet 1998) recently reported on tests comparing three FT fuels with two conventional diesel fuels (a \#2D and a "pseudo-CARB" reference fuel [PRF]). The properties of the different fuels are shown in Table 18. A prototype 1991 DDC Series $60 \mathrm{HD}$ diesel engine was used in the laboratory tests. The characteristics of this engine are listed in Table 19. No attempt was made to fine tune the engines or adjust the fuel flow rates to compensate for any inherent differences in the fuel properties. Table 20 provides the results of the hot-start transient emission measurements.

The fuel use and changes in emissions that would result from replacing diesel with FTD were estimated by using the following equations.

$$
\mathrm{F}_{\mathrm{FT}}=\mathrm{F}_{\mathrm{D}} \times \mathrm{LHV}_{\mathrm{D}} / \mathrm{LHV}_{\mathrm{FT}}
$$

and $\Delta \varepsilon=\left(\right.$ emission factor for FT)/(emission factor for diesel) $\times \varepsilon_{\mathrm{D}}$

The parameter values used in Equations 26 and 27 are given in Table 21. The results of the calculations are listed in Table 22. As the table shows, use of FTD would result in a net reduction of all end-use emissions except $\mathrm{CO}_{2}$.

\subsection{Scenario 4}

Scenario 4 assumes that DME would replace diesel in CI engines. DME is a light fuel, similar to propane. It is a gas at temperatures above $-25^{\circ} \mathrm{C}$ and can be stored in the liquid state under modest pressure (its vapor pressure at $20^{\circ} \mathrm{C}$ is about $5 \mathrm{~atm}$ ). DME has an auto-ignition temperature slightly lower than that of diesel (allowing compression ignition at nearly the same compression ratio) and a slightly higher cetane number (providing good startability), which make it a good candidate for diesel substitution. However, currently available fuel injection systems are not suitable for DME. Although the existing propane infrastructure might be adaptable to DME, it would require substantial expansion if DME were to replace diesel. (See Hansen and Mikkelsen 1998 for a recent discussion of DME as a neat diesel fuel. Also see Table 23, derived from Fleisch and Meurer [1995] for a comparison of DME with several other fuels.)

The fuel use and changes in emissions that would result from replacing diesel with FTD were estimated by using the following equations:

$$
\mathrm{F}_{\mathrm{DME}}=\mathrm{F}_{\mathrm{D}} \times \mathrm{LHV}_{\mathrm{D}} / \mathrm{LHV}_{\mathrm{DME}}
$$

and $\Delta \varepsilon=($ emission factor for DME $) /($ emission factor for diesel $) \times \varepsilon_{\mathrm{D}}$.

The parameter values used in these equations are listed in Table 20 (DME data from Fleisch 1995). The results of the calculations are provided in Table 24. With the exception of $\mathrm{CH}_{4}$ and $\mathrm{CO}$, all end-use emissions would be reduced by substituting DME for diesel. The reduction in ROG associated with the use of DME would be similar to that for FTD, but the reduction in $\mathrm{NO}_{\mathrm{x}}$ 
Table 18 Test Fuel Properties

\begin{tabular}{|c|c|c|c|c|c|}
\hline Test & Method & \#2 Diesel & $\begin{array}{l}\text { Fischer- } \\
\text { Tropsch } \\
\text { Formula } 1\end{array}$ & $\begin{array}{c}\text { Fischer- } \\
\text { Tropsch } \\
\text { Formula } 2\end{array}$ & $\begin{array}{l}\text { Fischer- } \\
\text { Tropsch } \\
\text { Formula } 3\end{array}$ \\
\hline Distillation & D86 & & & & \\
\hline $\operatorname{IBP}\left({ }^{\circ} \mathrm{F}\right)$ & & 376 & 338 & 348 & 382 \\
\hline $10 \%$ evaporation & & 438 & 427 & 394 & 448 \\
\hline $50 \%$ evaporation & & 501 & 590 & 458 & 546 \\
\hline $90 \%$ evaporation & & 587 & 646 & 536 & 620 \\
\hline $100 \%$ total evaporation & & 651 & 672 & 562 & 640 \\
\hline Cetane number & D613 & 45.5 & $>74.0$ & $>74.0$ & 74.0 \\
\hline \multirow[t]{2}{*}{ Cetane index } & D976 & 47.5 & 80.5 & 72.9 & 77.2 \\
\hline & D4737 & & 94.1 & 77.9 & 87.6 \\
\hline API gravity & D1298 (@60º) & 36.0 & 49.1 & 52.5 & 49.1 \\
\hline Density (g/mL) & $\left(@ 15^{\circ} \mathrm{C}\right)$ & & 0.7832 & 0.7688 & 0.7830 \\
\hline Specific gravity & & 0.8447 & 0.7835 & 0.7690 & 0.7833 \\
\hline Sulfur (wt. \%) & & 0.033 & $<0.001$ & $<0.001$ & $<0.001$ \\
\hline Flash point $\left({ }^{\circ} \mathrm{C}\right)$ & & 70.6 & 60 & 62.2 & 80.6 \\
\hline Cloud point $\left({ }^{\circ} \mathrm{C}\right)$ & & -16.6 & 8 & -23 & -12 \\
\hline Pour point $\left({ }^{\circ} \mathrm{C}\right)$ & & -26.0 & 11 & -20 & -16 \\
\hline Viscosity & $\begin{array}{c}\left(@ 40^{\circ} \mathrm{C},\right. \\
\text { Centistokes })\end{array}$ & 2.75 & 3.85 & 1.58 & 2.66 \\
\hline
\end{tabular}

\section{Table 19 Characteristics of Prototype 1991 DDC Series 60 Heavy-Duty Diesel Engine}

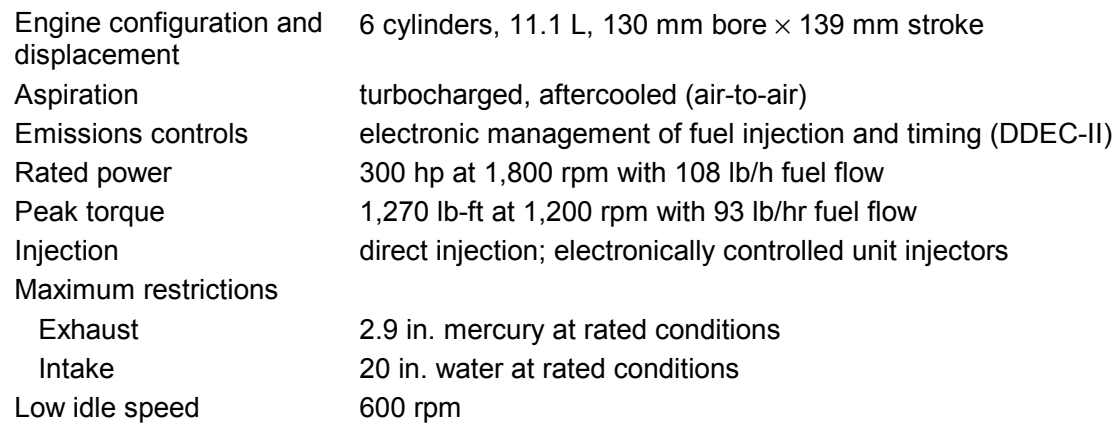


Table 20 Summary of Hot-Start Transient Emissions from a Prototype 1991 DDC Series 60

\begin{tabular}{|c|c|c|c|c|c|c|}
\hline \multirow[b]{2}{*}{ Parameter } & \multicolumn{6}{|c|}{ Hot-Start Transient Emissions (g/hp-h) } \\
\hline & $\mathrm{HC}$ & $\mathrm{CO}$ & $\mathrm{NO}_{\mathbf{x}}$ & PM & Sulfate & $\begin{array}{c}\text { Soluble Organic } \\
\text { Fraction }\end{array}$ \\
\hline Overall Mean - \#2 Diesel & 0.6142 & 1.9483 & 4.2318 & 0.1715 & 0.00313 & 0.0605 \\
\hline Standard deviation & 0.0187 & 0.0333 & 0.0201 & 0.0010 & 0.00022 & 0.0046 \\
\hline Coefficient of variance (\%) & 3.1 & 1.7 & 0.5 & 0.6 & 6.9 & 7.7 \\
\hline Overall Mean - FT Formula 1 & 0.3313 & 1.0733 & 3.8457 & 0.1253 & 0.00050 & 0.0322 \\
\hline Standard deviation & 0.0274 & 0.0352 & 0.0536 & 0.0010 & 0.00017 & 0.0040 \\
\hline Coefficient of variance (\%) & 8.3 & 3.3 & 1.4 & 0.8 & 33.5 & 12.3 \\
\hline Overall Mean - FT Formula 2 & 0.4578 & 1.0233 & 3.9490 & 0.1168 & 0.00020 & 0.0385 \\
\hline Standard deviation & 0.0054 & 0.0133 & 0.0010 & 0.0013 & 0.00008 & 0.0031 \\
\hline Coefficient of variance $(\%)$ & 1.2 & 1.3 & 0.0 & 1.1 & 40.8 & 8.1 \\
\hline Overall Mean - FT Formula 3 & 0.3608 & 1.0798 & 3.8455 & 0.1233 & 0.00068 & 0.0320 \\
\hline Standard deviation & 0.0316 & 0.0223 & 0.0101 & 0.0017 & 0.00029 & 0.0032 \\
\hline Coefficient of variance (\%) & 8.8 & 2.1 & 0.3 & 1.4 & 42.6 & 9.9 \\
\hline
\end{tabular}

would be substantially greater. The $\mathrm{CO}_{2}$ emissions would be lower, consistent with the lower carbon weight fraction of DME.

\subsection{Changes in Energy Use and Emissions from Production of Alternative Fuels}

To this point, only the changes resulting from the combustion of fuels have been considered. In this section, we discuss changes in fuel production, including the energy and emissions associated with the production of the feedstocks. Because energy consumption and emission factors for the production of various fuels have already been reported by Wang (1996) for use in the GREET model, those factors will be used here. The results for the base case and the four control scenarios for the year 2010 are provided in Tables 25 through 29. The base case results, shown in Table 25, are the total production energy use and emissions. The values for Scenarios 1 through 4 shown in Tables 26 through 29 are the increases in energy use and emissions caused by the production of the alternative fuels.

Several points are worth noting. First, the production energy is significantly greater for all four control scenario fuels than for the base case scenario. Second, Scenario 2 requires the least additional expenditure of energy; Scenario 3 (use of FTD) requires the greatest [Note: This conclusion is based on energy intensity for a straight-run FTD conversion plant using flared gas in conjunction with petroleum extraction. Either waste energy $/ \mathrm{CO}_{2}$ recovery modules or $\mathrm{CO}_{2}$ sequestration added to such a plant would significantly reduce both the input energy required and 
Table 21 Comparisons of Alternative Fuel Properties to California Standards ${ }^{a}$

\begin{tabular}{|c|c|c|c|c|c|c|c|c|}
\hline \multirow[b]{2}{*}{ Parameter } & \multicolumn{8}{|c|}{ Emission Factors in (g/bhp-h) } \\
\hline & ROG & CO & $\mathrm{NO}_{\mathrm{x}}$ & $\mathrm{CO}_{2}$ & $\mathrm{PM}_{10}$ & $\mathrm{so}_{\mathrm{x}}$ & $\mathrm{N}_{2} \mathrm{O}$ & $\mathrm{CH}_{4}$ \\
\hline $\begin{array}{l}\text { California ultra-low emission } \\
\text { vehicle (ULEV) standards }\end{array}$ & $N A^{b}$ & 7.200 & 2.000 & NA & 0.050 & NA & NA & NA \\
\hline $\begin{array}{l}\text { California } \mathrm{HD} \mathrm{Cl} \text { and urban bus } \\
\text { standards }\end{array}$ & 1.200 & 15.500 & 4.000 & NA & 0.050 & NA & NA & NA \\
\hline $\begin{array}{l}\text { DME emission factors - Navistar } \\
\text { T444 V8 tk. Engine }\end{array}$ & 0.168 & 3.200 & 2.190 & 176.73 & 0.040 & 0 & 0.077 & 0.042 \\
\hline $\begin{array}{l}\text { Diesel emission factors } \\
\text { (certification tests in U.S.) }\end{array}$ & 0.250 & 1.950 & 4.230 & 185.81 & 0.080 & 0.030 & 0.077 & 0.012 \\
\hline $\begin{array}{l}\text { Ratio of DME to diesel emission } \\
\text { factors }\end{array}$ & 0.672 & 1.641 & 0.518 & 0.95 & 0.500 & 0 & 1 & 3.511 \\
\hline $\begin{array}{l}\text { FTD emission factors }-1991 \text { series } \\
60 \text { DDC engine }\end{array}$ & 0.383 & 1.059 & 3.880 & 188.47 & 0.122 & 0 & 0.021 & 0.029 \\
\hline $\begin{array}{l}\text { \#2D emission factors }-1991 \text { series } \\
60 \text { DDC engine }\end{array}$ & 0.610 & 1.950 & 4.230 & 184.43 & 0.170 & 0.030 & 0.021 & 0.029 \\
\hline $\begin{array}{l}\text { Ratio of FTD to diesel emission } \\
\text { factors }\end{array}$ & 0.628 & 0.543 & 0.917 & 1.02 & 0.716 & 0 & 1 & 1 \\
\hline
\end{tabular}

a Notes:

Ratio of LHV (Btu/lb) of FTD to \#2D = 1.005

Ratio of LHV (Btu/lb) of DME to \#2D $=0.655$

Ratio of LHV (Btu/lb) of biodiesel to \#2D = 0.892

Ratio of LHV (Btu/lb) of LNG to \#2D = 1.155

$\mathrm{DME}=\mathrm{CO}_{3}-\mathrm{O}-\mathrm{CO}_{3}, \mathrm{MW}=46, \% \mathrm{C}=52.2$

Ratio of FTD to diesel fuel masses to produce equal torque curves is about 1.1; assume same ratio for energies.

The "well-to-wheel" $\mathrm{CO}_{2}$ emissions for DME are about $2 \%$ greater than for diesel.

The "well-to-wheel" $\mathrm{CO}_{2}$ emissions for LNG are about $4 \%$ greater than for diesel.

The "well-to-wheel" $\mathrm{CO}_{2}$ emissions for LPG are about $20 \%$ greater than for diesel

The ratio of volume of DME to volume of diesel containing same energy is 1.8 (Hansen and Mikkelsen 1998).

The ratio of volume of propane to volume of diesel containing same energy is 1.52 .

b $\mathrm{NA}=$ not applicable. 
Table 22 Estimated Increases in Energy and Emissions Under Scenario 3, 2010

\begin{tabular}{|c|c|c|c|c|c|c|c|c|c|c|c|}
\hline \multirow[b]{2}{*}{ Source Category } & \multirow[b]{2}{*}{$\begin{array}{c}\text { Added Daily } \\
\text { Energy } \\
\left(10^{6} \text { Btu }\right) \\
\end{array}$} & \multirow[b]{2}{*}{$\begin{array}{c}\text { Added } \\
\text { Fuel/Day } \\
\left(10^{3} \text { gal FTD }\right) \\
\end{array}$} & \multicolumn{9}{|c|}{ Daily Emissions (tons/day) due to Combustion } \\
\hline & & & ROG & $\mathrm{co}$ & $\mathrm{NO}_{\mathrm{x}}$ & $\mathrm{CO}_{2}\left(10^{3}\right)$ & $\mathrm{PM}_{10}$ & $\mathrm{SO}_{\mathrm{x}}$ & $\mathrm{N}_{2} \mathrm{O}^{\mathrm{b}}$ & $\mathrm{CH}_{4}{ }^{\mathrm{b}}$ & $\begin{array}{c}\text { GHGs } \\
\left(\mathrm{CO}_{2} \text {-equivalent }\right) \\
\left(10^{3}\right) \\
\end{array}$ \\
\hline \multicolumn{12}{|l|}{ On-Road Mobile Sources } \\
\hline Autos & 0 & 15.316 & -0.089 & -0.841 & -0.067 & 0.003 & -0.045 & -0.036 & 0 & 0 & 0.003 \\
\hline LD trucks & 0 & 3.559 & -0.019 & -0.155 & -0.013 & 0.001 & -0.011 & -0.008 & 0 & 0 & 0.001 \\
\hline MD trucks & 0 & $1,850.703$ & -0.539 & -26.078 & -3.211 & 0.425 & -0.811 & -5.323 & 0 & 0 & 0.425 \\
\hline HD trucks & 0 & $6,635.922$ & -13.550 & -156.892 & -29.025 & 1.517 & -4.117 & -18.831 & 0 & 0 & 1.517 \\
\hline Buses & 0 & 162.637 & -0.714 & -0.836 & -1.243 & 0.037 & -0.026 & -0.477 & 0 & 0 & 0.037 \\
\hline Subtotal & 0 & $8,668.136$ & -14.910 & -184.803 & -33.560 & 1.984 & -5.010 & -24.676 & 0 & 0 & 1.984 \\
\hline \multicolumn{12}{|l|}{ Off-Road Mobile Sources } \\
\hline Trains (locomotives) & 0 & 856.211 & -2.601 & -9.598 & .11 .582 & 0.196 & -0.851 & -21.852 & 0 & 0 & 0.196 \\
\hline Vessels & 0 & 747.262 & -3.068 & -3.584 & -2.678 & 0.171 & -0.961 & -42.560 & 0 & 0 & 0.171 \\
\hline Farm equipment (diesel) & 0 & 613.546 & -4.219 & -16.904 & -9.645 & 0.139 & -2.110 & -15.341 & 0 & 0 & 0.139 \\
\hline LD non-farm equipment (diesel) & 0 & 140.854 & -0.957 & -3.867 & -2.082 & 0.032 & -0.465 & -3.526 & 0 & 0 & 0.032 \\
\hline $\begin{array}{l}\text { HD non-farm equipment } \\
\text { (diesel) }\end{array}$ & 0 & 969.787 & -3.555 & -12.092 & -10.279 & 0.222 & -1.648 & -24.605 & 0 & 0 & 0.222 \\
\hline Refrigeration units (diesel) & 0 & 72.863 & -0.619 & -2.694 & -1.106 & 0.016 & -0.289 & -1.814 & 0 & 0 & 0.016 \\
\hline Subtotal & 0 & $3,400.524$ & -15.020 & -48.739 & -37.372 & 0.777 & -6.324 & -109.698 & 0 & 0 & 0.777 \\
\hline Stationary Sources & 0 & 157.984 & -0.844 & -6.235 & -3.303 & 0.036 & -1.151 & -3.813 & 0 & 0 & 0.036 \\
\hline Total - All Sources & 0 & $12,226.645$ & -30.773 & -239.777 & .74 .235 & 2.796 & -12.485 & 138.187 & 0 & 0 & 2.796 \\
\hline
\end{tabular}

a Efficiency of engines burning diesel and FTD assumed to be the same after proper tuning.

b Emissions factors were assumed to be the same for diesel and for FTD. 
Table 23 Combustion-Relevant Properties of DME Compared to Diesel and Other Alternative Fuels

\begin{tabular}{|c|c|c|c|c|c|}
\hline Property & DME & Diesel Fuel & Methanol & Ethanol & $\begin{array}{c}\text { CNG } \\
\text { (Methane) }\end{array}$ \\
\hline Chemical structure & $\mathrm{CH}_{3}-\mathrm{O}-\mathrm{CH}_{3}$ & $N A^{a}$ & $\mathrm{CH}_{3}-\mathrm{OH}$ & $\mathrm{CH}_{3}-\mathrm{CH}_{2}-\mathrm{OH}$ & $\mathrm{CH}_{4}$ \\
\hline $\begin{array}{l}\text { Lower calorific value } \\
(\mathrm{MJ} / \mathrm{kg})\end{array}$ & 27.6 & 42.5 & 19.5 & 25.0 & 50.0 \\
\hline Density $(\mathrm{g} / \mathrm{mL})$ & 0.66 & 0.84 & 0.79 & 0.81 & NA \\
\hline Cetane Number & $>55$ & $40-55$ & 5 & 8 & NA \\
\hline $\begin{array}{l}\text { Autoignition temperature } \\
\left({ }^{\circ} \mathrm{C}\right)\end{array}$ & 235 & 250 & 450 & 420 & 650 \\
\hline Octane number & NA & NA & 111 & 108 & 130 \\
\hline Stoichiometric air/fuel ratio & 9.0 & 14.6 & 6.5 & 9 & 17.2 \\
\hline Boiling Point $\left({ }^{\circ} \mathrm{C}\right)$ & -25 & $180-370$ & 65 & 78 & -162 \\
\hline Heat of evaporation $(\mathrm{kJ} / \mathrm{kg})$ & $\begin{array}{l}460\left(-20^{\circ}\right), 410 \\
\left(20^{\circ}\right)\end{array}$ & 250 & 1110 & 904 & NA \\
\hline $\begin{array}{l}\text { Explosion limits (\% gas in } \\
\text { air) }\end{array}$ & $3.4-18$ & $0.6-6.5$ & $5.5-26$ & $3.5-15$ & $5.0-15$ \\
\hline Percent weight carbon & 52.2 & 86.0 & 37.5 & 52.2 & 75.0 \\
\hline Percent weight hydrogen & 13.0 & 14.0 & 12.5 & 13.0 & 25.0 \\
\hline Percent weight oxygen & 34.8 & 0 & 50.0 & 34.8 & 0 \\
\hline
\end{tabular}

a $\mathrm{NA}=$ not applicable.

the GHGs emitted during FTD production]. Third, the GHG emissions are greater for all four control scenarios. Scenario 2 yields the smallest increase. With respect to precursors to ozone formation, only FTD and DME production result in reduced ROG emissions, but both of these scenarios increase $\mathrm{NO}_{\mathrm{x}}$ emissions.

\subsection{Changes in Energy Use and Emissions from Production and Combustion of Alternative Fuels}

Tables 30 through 33 show the changes in energy use and emissions caused by both fuel production and combustion for the four control scenarios.

Changes in energy consumption in the year 2010 resulting from production and combustion are summarized in Table 34. This table shows that the combined additional energy associated with combustion and production is lowest for Scenario 2. The table also shows that, although no additional energy is required for combustion of either FTD or DME, the production energy requirement for these alternative fuels is quite substantial (see Section 3.7 concerning possible alternative FTD plant factor assumptions).

Changes in $\mathrm{NO}_{\mathrm{x}}$ emissions are summarized in Table 35. The table shows that Scenarios 1 and 4 yield the greatest reductions in $\mathrm{NO}_{\mathrm{x}}$ emissions. Use of FTD (Scenario 3) produces almost no change in $\mathrm{NO}_{\mathrm{x}}$ emissions. 
Table 24 Estimated Increases in Energy and Emissions under Scenario 4, 2010

\begin{tabular}{|c|c|c|c|c|c|c|c|c|c|c|c|}
\hline \multirow[b]{2}{*}{ Source Category } & \multirow[b]{2}{*}{$\begin{array}{c}\text { Added Daily } \\
\text { Energy } \\
\left(10^{6} \text { Btu }\right)\end{array}$} & \multirow[b]{2}{*}{$\begin{array}{c}\text { Added } \\
\text { Fuel/Day } \\
\left(10^{3} \text { gal DME }\right)\end{array}$} & \multicolumn{9}{|c|}{ Changes in Emissions (tons/day) due to Combustion } \\
\hline & & & ROG & co & $\mathrm{NO}_{\mathrm{x}}$ & $\mathrm{CO}_{2}\left(10^{3}\right)$ & $\mathrm{PM}_{10}$ & $\mathrm{SO}_{\mathbf{x}}$ & $\mathrm{N}_{2} \mathrm{O}^{\mathrm{b}}$ & $\mathrm{CH}_{4}$ & $\begin{array}{c}\text { GHGs } \\
\begin{array}{c}\left(\mathrm{CO}_{2} \text {-equivalent }\right) \\
\left(10^{3}\right)\end{array} \\
\end{array}$ \\
\hline \multicolumn{12}{|l|}{ On-Road Mobile Sources } \\
\hline Autos & 0 & 27 & -0.08 & 1.18 & -0.39 & -0.01 & -0.08 & -0.04 & 0 & 0.02 & -0.01 \\
\hline LD trucks & 0 & 6 & -0.02 & 0.22 & -0.08 & 0 & -0.02 & -0.01 & 0 & 0.00 & 0.00 \\
\hline MD trucks & 0 & 3,225 & -0.48 & 36.58 & -18.72 & -0.95 & -1.43 & -5.32 & 0 & 0.95 & -0.93 \\
\hline HD trucks & 0 & 11,563 & -11.96 & 220.06 & -169.20 & -3.38 & -7.26 & -18.83 & 0 & 2.68 & -3.32 \\
\hline Buses & 0 & 283 & -0.63 & 1.17 & -7.25 & -0.08 & -0.05 & -0.48 & 0 & 0.17 & -0.08 \\
\hline Subtotal & 0 & 15,104 & -13.16 & 259.21 & -195.64 & -4.42 & -8.84 & -24.68 & 0 & 3.82 & -4.34 \\
\hline \multicolumn{12}{|l|}{ Off-Road Mobile Sources } \\
\hline Trains (locomotives) & 0 & 1,492 & -2.30 & 13.46 & -67.52 & -0.44 & -1.50 & -21.85 & 0 & 0.36 & -0.43 \\
\hline Vessels & 0 & 1,302 & -2.71 & 5.03 & -15.61 & -0.38 & -1.69 & -42.56 & 0 & 0.31 & -0.38 \\
\hline Farm equipment (diesel) & 0 & 1,069 & -3.72 & 23.71 & -56.23 & -0.31 & -3.72 & -15.34 & 0 & 0.27 & -0.30 \\
\hline LD Non-farm equipment (diesel) & 0 & 245 & -0.84 & 5.42 & -12.14 & -0.07 & -0.82 & -3.53 & 0 & 0.08 & -0.07 \\
\hline HD Non-farm equipment (diesel) & 0 & 1,690 & -3.14 & 16.96 & -59.92 & -0.49 & -2.91 & -24.61 & 0 & 0.43 & -0.49 \\
\hline Refrigeration units (diesel) & 0 & 127 & -0.55 & 3.78 & -6.44 & -0.04 & -0.51 & -1.81 & 0 & 0.08 & -0.03 \\
\hline Subtotal & 0 & 5,925 & -13.26 & 68.36 & -217.87 & -1.73 & -11.15 & -109.70 & 0 & 1.52 & -1.70 \\
\hline Stationary Sources & 0 & 275 & -0.74 & 8.74 & -19.26 & -0.08 & -2.03 & -3.81 & 0 & 0.00 & -0.08 \\
\hline Total - All Sources & 0 & 21,305 & -27.16 & 336.31 & -432.77 & -6.23 & -22.02 & -138.19 & 0 & 5.34 & -6.12 \\
\hline
\end{tabular}

a Efficiency of engines burning diesel and FTD were assumed equal (after proper tuning).

b Emissions factors were assumed to be the same for diesel and FTD. 
Table 25 Energy and Emissions from Production of Baseline Diesel Fuel, 2010

\begin{tabular}{|c|c|c|c|c|c|c|c|c|c|c|}
\hline \multirow[b]{2}{*}{ Source Category } & \multirow[b]{2}{*}{$\begin{array}{c}\text { Added Daily } \\
\text { Energy } \\
\left(10^{6} \text { Btu }\right) \\
\end{array}$} & \multicolumn{9}{|c|}{ Changes in Daily Emissions (ton/d) } \\
\hline & & ROG & Co & $\mathrm{NO}_{\mathrm{x}}$ & $\mathrm{CO}_{2}$ & $\mathbf{P M}_{10}$ & SO $_{x}$ & $\mathrm{~N}_{2} \mathrm{O}$ & $\mathrm{CH}_{4}$ & $\begin{array}{c}\text { GHGs } \\
\left(\mathrm{CO}_{2} \text {-equivalent }\right) \\
\left(10^{3}\right)\end{array}$ \\
\hline \multicolumn{11}{|l|}{ On-Road Mobile Sources } \\
\hline Autos & 282 & 0.02 & 0.04 & 0.05 & 0.02 & 0 & 0.03 & 0 & 0.15 & 0.03 \\
\hline LD trucks & 66 & 0 & 0.01 & 0.01 & 0.01 & 0 & 0.01 & 0 & 0.04 & 0.01 \\
\hline MD trucks & 34,134 & 2.24 & 4.90 & 5.75 & 2.78 & 0.56 & 3.54 & 0.31 & 18.38 & 3.26 \\
\hline HD trucks & 122,391 & 8.04 & 17.58 & 20.61 & 9.98 & 2.01 & 12.68 & 1.09 & 65.92 & 11.70 \\
\hline Buses & 3,000 & 0.20 & 0.43 & 0.51 & 0.24 & 0.05 & 0.31 & 0.03 & 1.62 & 0.29 \\
\hline Subtotal & 159,873 & 10.50 & 22.97 & 26.92 & 13.03 & 2.62 & 16.56 & 1.43 & 86.11 & 15.28 \\
\hline \multicolumn{11}{|l|}{ Off-Road Mobile Sources } \\
\hline Trains (locomotives) & 15,792 & 1.04 & 2.27 & 2.66 & 1.29 & 0.26 & 1.64 & 0.14 & 8.51 & 1.51 \\
\hline Vessels & 13,782 & 0.91 & 1.98 & 2.32 & 1.12 & 0.23 & 1.43 & 0.12 & 7.42 & 1.32 \\
\hline Farm equipment (diesel) & 11,316 & 0.74 & 1.63 & 1.91 & 0.92 & 0.19 & 1.17 & 0.10 & 6.09 & 1.08 \\
\hline LD Non-farm equipment (diesel) & 2,598 & 0.17 & 0.37 & 0.44 & 0.21 & 0.04 & 0.27 & 0.02 & 1.40 & 0.25 \\
\hline HD Non-farm equipment (diesel) & 17,886 & 1.17 & 2.57 & 3.01 & 1.46 & 0.29 & 1.85 & 0.16 & 9.63 & 1.71 \\
\hline Refrigeration units (diesel) & 1,344 & 0.09 & 0.19 & 0.23 & 0.11 & 0.02 & 0.14 & 0.01 & 0.72 & 0.13 \\
\hline Subtotal & 62,718 & 4.12 & 9.01 & 10.56 & 5.11 & 1.03 & 6.50 & 0.56 & 33.78 & 6.00 \\
\hline Stationary Sources & 2,914 & 0.19 & 0.42 & 0.49 & 0.24 & 0.05 & 0.30 & 0.03 & 1.57 & 0.28 \\
\hline Total - All Sources & 225,505 & 14.81 & 32.40 & 37.97 & 18.38 & 3.70 & 23.36 & 2.02 & 121.45 & 21.56 \\
\hline
\end{tabular}


Table 26 Changes in Energy and Emissions Caused by Production of Control Scenario 1 Fuels, 2010

\begin{tabular}{|c|c|c|c|c|c|c|c|c|c|c|}
\hline \multirow[b]{2}{*}{ Source Category } & \multirow[b]{2}{*}{$\begin{array}{c}\text { Added Daily } \\
\text { Energy } \\
\left(10^{6} \text { Btu }\right)\end{array}$} & \multicolumn{9}{|c|}{ Changes in Daily Emissions (ton/d) } \\
\hline & & ROG & Co & $\mathrm{NO}_{\mathbf{x}}$ & $\mathrm{CO}_{2}$ & $\mathrm{PM}_{10}$ & $\mathrm{SO}_{\mathrm{x}}$ & $\mathrm{N}_{2} \mathrm{O}$ & $\mathrm{CH}_{4}$ & $\begin{array}{c}\text { GHGs } \\
\left(\mathrm{CO}_{2} \text {-equivalent }\right) \\
\left(10^{3}\right) \\
\end{array}$ \\
\hline \multicolumn{11}{|l|}{ On-Road Mobile Sources } \\
\hline Autos & 241 & 0.02 & 0.01 & 0.03 & 0.02 & 0 & 0.03 & 0 & 0.04 & 0.02 \\
\hline LD trucks & 56 & 0 & 0 & 0.01 & 0 & 0 & 0.01 & 0 & 0.01 & 0 \\
\hline MD trucks & 44,294 & 3.73 & 2.74 & 5.65 & 3.46 & 0.68 & 4.60 & 0.39 & 10.23 & 3.80 \\
\hline HD trucks & 246,478 & 20.05 & 18.35 & 32.99 & 19.40 & 3.83 & 25.59 & 2.16 & 68.65 & 21.51 \\
\hline Buses & 5,384 & 0.44 & 0.39 & 0.71 & 0.42 & 0.08 & 0.56 & 0.05 & 1.44 & 0.47 \\
\hline Subtotal & 296,454 & 24.25 & 21.49 & 39.38 & 23.31 & 4.59 & 30.78 & 2.60 & 80.36 & 25.80 \\
\hline \multicolumn{11}{|l|}{ Off-Road Mobile Sources } \\
\hline Trains (locomotives) & 22,546 & 2.45 & 5.36 & 11.39 & 1.07 & 1.27 & 0.55 & 1.27 & 20.78 & 1.90 \\
\hline Vessels & 19,677 & 2.14 & 4.67 & 9.94 & 0.93 & 1.11 & 0.48 & 1.11 & 18.13 & 1.66 \\
\hline Farm equipment (diesel) & 12,272 & 1.05 & 0.67 & 1.52 & 0.96 & 0.19 & 1.27 & 0.11 & 2.51 & 1.04 \\
\hline LD Non-farm equipment (diesel) & 2,168 & 0.19 & 0.09 & 0.25 & 0.17 & 0.03 & 0.23 & 0.02 & 0.34 & 0.18 \\
\hline HD Non-farm equipment (diesel) & 19,398 & 1.66 & 1.06 & 2.41 & 1.51 & 0.30 & 2.01 & 0.17 & 3.97 & 1.65 \\
\hline Refrigeration units (diesel) & 1,121 & 0.10 & 0.05 & 0.13 & 0.09 & 0.02 & 0.12 & 0.01 & 0.18 & 0.09 \\
\hline Subtotal & 77,182 & 7.60 & 11.90 & 25.64 & 4.72 & 2.92 & 4.67 & 2.68 & 45.91 & 6.52 \\
\hline Stationary Sources & 4,160 & 0.45 & 0.99 & 2.10 & 0.20 & 0.23 & 0.10 & 0.23 & 3.83 & 0.35 \\
\hline Total - All Sources & 377,796 & 32.30 & 34.38 & 67.13 & 28.23 & 7.75 & 35.55 & 5.52 & 130.10 & 32.67 \\
\hline
\end{tabular}


Table 27 Changes in Energy and Emissions Caused by Production of Control Scenario 2 Fuels, 2010

\begin{tabular}{|c|c|c|c|c|c|c|c|c|c|c|}
\hline \multirow[b]{2}{*}{ Source Category } & \multirow[b]{2}{*}{$\begin{array}{c}\text { Added Daily } \\
\text { Energy } \\
\left(10^{6} \text { Btu }\right)\end{array}$} & \multicolumn{9}{|c|}{ Changes in Daily Emissions (ton/d) } \\
\hline & & ROG & co & $\mathrm{NO}_{\mathbf{x}}$ & $\mathrm{CO}_{2}$ & $\mathbf{P M}_{10}$ & so $_{x}$ & $\mathrm{~N}_{\mathbf{2}} \mathrm{O}$ & $\mathrm{CH}_{4}$ & $\begin{array}{c}\text { GHGs } \\
\left.\text { ( } \mathrm{CO}_{2} \text {-equivalent }\right) \\
\left(10^{3}\right)\end{array}$ \\
\hline \multicolumn{11}{|l|}{ On-Road Mobile Sources } \\
\hline Autos & 241 & 0.02 & 0.01 & 0.03 & 0.02 & 0 & 0.03 & 0 & 0.04 & 0.02 \\
\hline LD trucks & 56 & 0 & 0 & 0.01 & 0 & 0 & 0.01 & 0 & 0.01 & 0 \\
\hline MD trucks & $-15,781$ & 1.88 & 1.70 & 5.77 & 2.39 & 0.47 & 4.16 & 0.43 & 15.83 & 2.86 \\
\hline HD trucks & 49,374 & 2.74 & 8.64 & 34.11 & 9.39 & 1.82 & 21.43 & 2.59 & 121.11 & 12.74 \\
\hline Buses & 4,469 & 0.03 & 0.40 & 1.41 & 0.34 & 0.07 & 0.84 & 0.11 & 5.36 & 0.49 \\
\hline Subtotal & 38,358 & 4.68 & 10.75 & 41.33 & 12.15 & 2.35 & 26.46 & 3.13 & 142.34 & 16.11 \\
\hline \multicolumn{11}{|l|}{ Off-Road Mobile Sources } \\
\hline Trains (locomotives) & 17,465 & 1.95 & 4.25 & 9.25 & 0.76 & 1.04 & 0.30 & 1.05 & 16.51 & 1.44 \\
\hline Vessels & 15,242 & 1.70 & 3.71 & 8.08 & 0.67 & 0.91 & 0.26 & 0.91 & 14.41 & 1.25 \\
\hline Farm equipment (diesel) & 12,272 & 1.05 & 0.67 & 1.52 & 0.96 & 0.19 & 1.27 & 0.19 & 7.21 & 1.88 \\
\hline LD Non-farm equipment (diesel) & 2,168 & 0.19 & 0.09 & 0.25 & 0.17 & 0.03 & 0.23 & .0 .12 & .7 .89 & -1.28 \\
\hline HD Non-farm equipment (diesel) & 19,398 & 1.66 & 1.06 & 2.41 & 1.51 & 0.30 & 2.01 & 0.32 & 12.88 & 3.23 \\
\hline Refrigeration units (diesel) & 1,121 & 0.10 & 0.05 & 0.13 & 0.09 & 0.02 & 0.12 & -0.54 & -32.88 & -5.77 \\
\hline Subtotal & 67,665 & 6.65 & 9.85 & 21.64 & 4.15 & 2.48 & 4.19 & 1.81 & 10.23 & 0.74 \\
\hline Stationary Sources & 3,222 & 0.36 & 0.78 & 1.71 & 0.14 & 0.19 & 0.05 & 0.22 & 4.62 & 0.54 \\
\hline Total - All Sources & 109,246 & 11.69 & 21.37 & 64.68 & 16.45 & 5.02 & 30.71 & 5.16 & 157.19 & 17.39 \\
\hline
\end{tabular}


Table 28 Changes in Energy and Emissions Caused by Production of Control Scenario 3 Fuel (FTD), 2010

\begin{tabular}{|c|c|c|c|c|c|c|c|c|c|c|}
\hline \multirow[b]{2}{*}{ Source Category } & \multirow[b]{2}{*}{$\begin{array}{c}\text { Added Daily } \\
\text { Energy } \\
\left(10^{6} \text { Btu }\right)\end{array}$} & \multicolumn{9}{|c|}{ Changes in Daily Emissions (ton/d) } \\
\hline & & ROG & $\mathrm{CO}$ & $\mathrm{NO}_{x}$ & $\mathrm{CO}_{2}$ & $\mathrm{PM}_{10}$ & $\mathrm{so}_{\mathrm{x}}$ & $\mathrm{N}_{2} \mathrm{O}$ & $\mathrm{CH}_{4}$ & $\begin{array}{c}\text { GHGs } \\
\left(\mathrm{CO}_{2} \text {-equivalent }\right) \\
\left(10^{3}\right)\end{array}$ \\
\hline \multicolumn{11}{|l|}{ On-Road Mobile Sources } \\
\hline Autos & 1380 & -0.01 & 0.04 & 0.06 & 0.10 & 0.00 & -0.01 & 0 & 0.07 & 0.10 \\
\hline LD trucks & 321 & 0 & 0.01 & 0.01 & 0.02 & 0 & 0 & 0 & 0.02 & 0.02 \\
\hline MD trucks & 166,747 & -1.17 & 5.14 & 7.17 & 11.52 & -0.02 & -1.29 & -0.15 & 8.10 & 11.64 \\
\hline HD trucks & 597,892 & -4.20 & 18.43 & 25.70 & 41.30 & -0.08 & -4.63 & -0.52 & 29.04 & 41.74 \\
\hline Buses & 14,653 & -0.10 & 0.45 & 0.63 & 1.01 & 0.00 & -0.11 & -0.01 & 0.71 & 1.02 \\
\hline Subtotal & 780,993 & -5.48 & 24.07 & 33.57 & 53.94 & -0.11 & -6.05 & -0.68 & 37.93 & 54.53 \\
\hline \multicolumn{11}{|l|}{ Off-Road Mobile Sources } \\
\hline Trains (locomotives) & 77,144 & -0.54 & 2.38 & 3.32 & 5.33 & -0.01 & -0.60 & -0.07 & 3.75 & 5.39 \\
\hline Vessels & 67,328 & -0.47 & 2.07 & 2.89 & 4.65 & -0.01 & -0.52 & -0.06 & 3.27 & 4.70 \\
\hline Farm equipment (diesel) & 55,280 & -0.39 & 1.70 & 2.38 & 3.82 & -0.01 & -0.43 & -0.05 & 2.69 & 3.86 \\
\hline LD Non-farm equipment (diesel) & 12,691 & -0.09 & 0.39 & 0.55 & 0.88 & 0 & -0.10 & -0.01 & 0.62 & 0.89 \\
\hline HD Non-farm equipment (diesel) & 87,377 & -0.61 & 2.69 & 3.76 & 6.04 & -0.01 & -0.68 & -0.08 & 4.24 & 6.10 \\
\hline Refrigeration units (diesel) & 6565 & -0.05 & 0.20 & 0.28 & 0.45 & 0 & -0.05 & -0.01 & 0.32 & 0.46 \\
\hline Subtotal & 306,385 & -2.15 & 9.44 & 13.17 & 21.16 & -0.04 & -2.37 & -0.27 & 14.88 & 21.39 \\
\hline Stationary Sources & 14,234 & -0.10 & 0.44 & 0.61 & 0.98 & 0 & -0.11 & -0.01 & 0.69 & 0.99 \\
\hline Total - All Sources & $1,101,612$ & -7.73 & 33.95 & 47.36 & 76.09 & -0.15 & -8.54 & -0.96 & 53.51 & 76.91 \\
\hline
\end{tabular}


Table 29 Changes in Energy and Emissions Caused by Production of Control Scenario 4 Fuel (DME), 2010

\begin{tabular}{|c|c|c|c|c|c|c|c|c|c|c|}
\hline \multirow[b]{2}{*}{ Source Category } & \multirow[b]{2}{*}{$\begin{array}{c}\text { Added Daily } \\
\text { Energy } \\
\left(10^{6} \mathrm{Btu}\right)\end{array}$} & \multicolumn{9}{|c|}{ Changes in Daily Emissions (ton/d) } \\
\hline & & ROG & Co & $\mathrm{NO}_{\mathbf{x}}$ & $\mathrm{CO}_{2}$ & $\mathrm{PM}_{10}$ & $\mathrm{SO}_{\mathrm{x}}$ & $\mathrm{N}_{2} \mathrm{O}$ & $\mathrm{CH}_{4}$ & $\begin{array}{c}\text { GHGs } \\
\left(\mathrm{CO}_{2} \text {-equivalent }\right) \\
\left(10^{3}\right) \\
\end{array}$ \\
\hline \multicolumn{11}{|l|}{ On-Road Mobile Sources } \\
\hline Autos & 937 & 0 & 0.03 & 0.04 & 0.04 & 0 & -0.01 & 0 & 0.07 & 0.04 \\
\hline LD trucks & 218 & 0 & 0.01 & 0.01 & 0.01 & 0 & 0.00 & 0 & 0.02 & 0.01 \\
\hline MD trucks & 113,223 & -0.52 & 3.28 & 4.49 & 4.92 & -0.12 & -1.42 & -0.15 & 7.91 & 5.03 \\
\hline HD trucks & 405,974 & -1.87 & 11.76 & 16.08 & 17.63 & -0.43 & -5.09 & -0.55 & 28.35 & 18.05 \\
\hline Buses & 9,950 & -0.05 & 0.29 & 0.39 & 0.43 & -0.01 & -0.12 & -0.01 & 0.69 & 0.44 \\
\hline Subtotal & 530,301 & -2.45 & 15.36 & 21.01 & 23.02 & -0.57 & -6.65 & -0.72 & 37.03 & 23.58 \\
\hline \multicolumn{11}{|l|}{ Off-Road Mobile Sources } \\
\hline Trains (locomotives) & 52,381 & -0.24 & 1.52 & 2.08 & 2.27 & -0.06 & -0.66 & -0.07 & 3.66 & 2.33 \\
\hline Vessels & 45,716 & -0.21 & 1.32 & 1.81 & 1.98 & -0.05 & -0.57 & -0.06 & 3.19 & 2.03 \\
\hline Farm equipment (diesel) & 37,536 & -0.17 & 1.09 & 1.49 & 1.63 & -0.04 & -0.47 & -0.05 & 2.62 & 1.67 \\
\hline LD Non-farm equipment (diesel) & 8,617 & -0.04 & 0.25 & 0.34 & 0.37 & -0.01 & -0.11 & -0.01 & 0.60 & 0.38 \\
\hline HD Non-farm equipment (diesel) & 59,330 & -0.27 & -0.06 & -0.74 & -0.08 & 4.14 & 2.64 & -0.08 & 4.14 & 2.64 \\
\hline Refrigeration units (diesel) & 4,458 & -0.02 & 0.00 & -0.06 & -0.01 & 0.31 & 0.20 & -0.01 & 0.31 & 0.20 \\
\hline Subtotal & 208,038 & -0.96 & 6.03 & 8.24 & 9.03 & -0.22 & -2.61 & -0.28 & 14.53 & 9.25 \\
\hline Stationary Sources & 9,665 & -0.040 & 0.28 & 0.38 & 0.42 & -0.01 & -0.12 & -0.01 & 0.67 & 0.43 \\
\hline Total - All Sources & 748,004 & -3.45 & 21.67 & 29.63 & 32.47 & -0.80 & -9.38 & -1.02 & 52.24 & 33.25 \\
\hline
\end{tabular}


Table 30 Net Changes in Energy and Emissions Caused by Production and Combustion of Control Scenario 1 Fuels, 2010

\begin{tabular}{|c|c|c|c|c|c|c|c|c|c|c|c|c|c|}
\hline \multirow[b]{2}{*}{ Source Category } & \multicolumn{4}{|c|}{ Changes in Daily Fuel Energy $\left(10^{6} \mathrm{Btu}\right)$} & \multicolumn{8}{|c|}{ Changes in Emissions (ton/day) } & \multirow{2}{*}{$\begin{array}{c}\text { GHGs } \\
\left(\mathrm{CO}_{2} \text {-equivalent }\right) \\
\left(10^{3}\right)\end{array}$} \\
\hline & Diesel & Gasoline & CNG & LNG & ROG & Co & $\mathrm{NO}_{\mathbf{x}}$ & $\mathrm{CO}_{2}\left(10^{3}\right)$ & $\mathrm{PM}_{10}$ & So $_{x}$ & $\mathrm{~N}_{2} \mathrm{O}$ & $\mathrm{CH}_{4}$ & \\
\hline \multicolumn{14}{|l|}{ On-Road Mobile Sources } \\
\hline Autos & $-2,105$ & 2,548 & & 0 & -0.02 & 0.09 & -0.08 & 0.01 & .0 .04 & 0.01 & 0.01 & 0.01 & 0.01 \\
\hline LD trucks & -489 & 592 & & 0 & 13.89 & 136.00 & 12.30 & 9.09 & -0.92 & 4.68 & 8.08 & 11.02 & 11.82 \\
\hline MD trucks & $-254,340$ & 381,835 & & 0 & 35.75 & 926.78 & 40.77 & 67.28 & -4.64 & 32.16 & 70.79 & 74.50 & 90.79 \\
\hline HD trucks & $-911,966$ & $1,795,880$ & & 0 & 1.28 & 36.84 & 7.12 & 2.68 & 0.19 & 0.94 & 2.55 & 1.69 & 3.51 \\
\hline Buses & $-22,351$ & 40,818 & & 0 & 50.89 & 1100.22 & 59.59 & 79.08 & -5.55 & 37.82 & 81.46 & 87.28 & 106.17 \\
\hline Subtotal & $-1,191,250$ & $2,221,673$ & & 0 & & & & & & & & & \\
\hline Off-Road Mobile Sources & & & & & 14.63 & 19.22 & -120.21 & -0.11 & 0.37 & -21.30 & 1.27 & 21.93 & 0.74 \\
\hline Trains (locomotives) & $-117,668$ & 0 & & 158,552 & 16.50 & 9.85 & -20.49 & -0.10 & 0.09 & .18 .59 & 1.11 & 19.14 & 0.65 \\
\hline Vessels & $-102,695$ & 0 & & 138,377 & -6.96 & 43.73 & -92.14 & 2.10 & -6.87 & -12.44 & 1.35 & 2.60 & 2.58 \\
\hline $\begin{array}{l}\text { Farm equipment } \\
\text { (diesel) }\end{array}$ & $-84,319$ & 114,842 & & 0 & 12.02 & 418.73 & -18.83 & -0.46 & -1.60 & -2.97 & 0.58 & 0.37 & -0.27 \\
\hline $\begin{array}{l}\text { LD non-farm equipment } \\
\text { (diesel) }\end{array}$ & $-19,357$ & 23,201 & & 0 & 2.45 & 268.08 & -115.50 & 2.95 & -5.41 & .19 .99 & 2.14 & 4.12 & 3.70 \\
\hline $\begin{array}{l}\text { HD non-farm equipment } \\
\text { (diesel) }\end{array}$ & $-133,277$ & 181,522 & & 0 & 5.88 & 215.09 & -10.08 & -0.23 & -0.99 & -1.53 & 0.14 & 0.22 & -0.19 \\
\hline $\begin{array}{l}\text { Refrigeration units } \\
\text { (diesel) }\end{array}$ & $-10,013$ & 12,002 & & 0 & 44.52 & 974.70 & -377.24 & 4.15 & -14.40 & -76.83 & 6.59 & 48.39 & 7.21 \\
\hline Subtotal & $-467,329$ & 331,566 & & 296,929 & 4.40 & 9.99 & -32.24 & 0.08 & -0.98 & -3.71 & 0.23 & 3.83 & 0.23 \\
\hline Stationary Sources & $-21,712$ & 0 & & 29,255 & 99.81 & 2084.91 & -349.89 & 83.31 & -20.94 & -42.72 & 88.29 & 139.50 & 113.60 \\
\hline Total - All Sources & $-1,680,291$ & $2,553,239$ & & 326,184 & 99.81 & 2084.91 & -349.89 & 83.31 & -20.94 & -42.72 & 88.29 & 139.50 & 113.60 \\
\hline
\end{tabular}


Table 31 Net Changes in Energy and Emissions Caused by Production and Combustion of Control Scenario 2 Fuels, 2010

\begin{tabular}{|c|c|c|c|c|c|c|c|c|c|c|c|c|c|}
\hline \multirow[b]{2}{*}{ Source Category } & \multicolumn{4}{|c|}{ Changes in Daily Fuel Energy $\left(10^{6}\right.$ Btu) } & \multicolumn{8}{|c|}{ Changes in Emissions (ton/day) } & \multirow{2}{*}{$\begin{array}{c}\text { GHGs } \\
\left(\mathrm{CO}_{2} \text {-equivalent }\right) \\
\left(10^{3}\right)\end{array}$} \\
\hline & Diesel & Gasoline & CNG & LNG & ROG & $\mathrm{CO}$ & $\mathrm{NO}_{x}$ & $\mathrm{CO}_{2}\left(10^{3}\right)$ & $\mathbf{P M}_{10}$ & $\mathrm{SO}_{\mathrm{x}}$ & $\mathrm{N}_{2} \mathrm{O}$ & $\mathrm{CH}_{4}$ & \\
\hline \multicolumn{14}{|l|}{ On-Road Mobile Sources } \\
\hline Autos & $-2,105$ & 2,548 & 0 & 0 & -0.02 & 0.51 & -0.52 & 0.03 & -0.14 & 0.02 & 0.03 & 0.05 & 0.04 \\
\hline LD trucks & -489 & 592 & 0 & 0 & -0.02 & 0.09 & -0.08 & 0.01 & -0.04 & 0.01 & 0.01 & 0.01 & 0.01 \\
\hline MD trucks & $-254,340$ & 234,003 & 18,352 & 0 & 7.65 & 70.81 & 1.17 & 0.05 & -1.61 & 2.27 & 5.11 & 23.58 & 2.13 \\
\hline HD trucks & $-911,966$ & 412,281 & 171,765 & 0 & -14.03 & -23.73 & -10.05 & 1.19 & -11.15 & 10.21 & 18.23 & 232.15 & 11.71 \\
\hline Buses & $-22,351$ & 2,431 & 7,469 & 0 & -1.29 & 1.24 & -3.80 & -0.60 & .0 .01 & 0.44 & 0.27 & 8.94 & -0.33 \\
\hline Subtotal & $-1,191,250$ & 651,855 & 197,586 & 0 & -7.70 & 48.92 & -13.28 & 0.67 & -12.96 & 12.95 & 23.65 & 264.73 & 13.56 \\
\hline \multicolumn{14}{|l|}{ Off-Road Mobile Sources } \\
\hline Trains (locomotives) & $-104,838$ & 0 & 0 & 127,345 & 7.45 & 104.34 & -44.45 & 0.14 & 0.78 & -12.81 & 1.04 & 51.36 & 1.54 \\
\hline Vessels & $-91,498$ & 0 & 0 & 111,141 & 8.19 & 41.09 & -4.34 & 0.12 & 0.61 & -25.28 & 0.91 & 44.82 & 1.34 \\
\hline $\begin{array}{l}\text { Farm equipment } \\
\text { (diesel) }\end{array}$ & $-84,319$ & 114,842 & 0 & 0 & -6.96 & 43.73 & -92.14 & 2.10 & -6.87 & -12.44 & 1.43 & 7.30 & 3.41 \\
\hline $\begin{array}{l}\text { LD non-farm equipment } \\
\text { (diesel) }\end{array}$ & $-19,357$ & 23,201 & 0 & 0 & 12.02 & 418.73 & -18.83 & -0.46 & -1.60 & -2.97 & 0.44 & -7.86 & -1.74 \\
\hline $\begin{array}{l}\text { HD non-farm equipment } \\
\text { (diesel) }\end{array}$ & $-133,277$ & 181,522 & 0 & 0 & 2.45 & 268.08 & -115.50 & 2.95 & -5.41 & -19.99 & 2.29 & 13.03 & 5.28 \\
\hline Subtotal & $-443,301$ & 331,566 & 0 & 238,486 & 29.02 & 1091.05 & -285.34 & 4.61 & -13.48 & .75 .02 & 5.71 & 75.82 & 3.78 \\
\hline Stationary Sources & 2,367 & & & 23,497 & 10.57 & 235.43 & 14.89 & 1.43 & 3.28 & 0.41 & 0.22 & 4.62 & 1.83 \\
\hline Total - All Sources & $-1,632,184$ & 983,421 & 197,586 & 261,983 & 31.90 & 1375.40 & -283.73 & 6.70 & -23.16 & -61.65 & 29.57 & 345.17 & 19.17 \\
\hline
\end{tabular}


Table 32 Net Changes in Energy and Emissions Caused by Production and Combustion of Control Scenario 3 Fuel (FTD), 2010

\begin{tabular}{|c|c|c|c|c|c|c|c|c|c|c|c|}
\hline \multirow[b]{2}{*}{ Source Category } & \multicolumn{2}{|c|}{$\begin{array}{c}\text { Changes in Daily Fuel } \\
\text { Energy }\left(10^{6} \mathrm{Btu}\right)\end{array}$} & \multicolumn{8}{|c|}{ Changes in Emissions (ton/day) } & \multirow{2}{*}{$\begin{array}{c}\text { GHGs } \\
\left(\mathrm{CO}_{2} \text {-equivalent }\right) \\
\left(10^{3}\right)\end{array}$} \\
\hline & Diesel & FT & ROG & CO & $\mathrm{NO}_{\mathrm{x}}$ & $\mathrm{CO}_{2}\left(10^{3}\right)$ & $\mathrm{PM}_{10}$ & $\mathrm{so}_{\mathrm{x}}$ & $\mathrm{N}_{2} \mathrm{O}$ & $\mathrm{CH}_{4}$ & \\
\hline \multicolumn{12}{|l|}{ On-Road Mobile Sources } \\
\hline Autos & $-2,105$ & 3,200 & -0.10 & -0.80 & -0.01 & 0.10 & -0.05 & -0.05 & 0.00 & 0.07 & 0.10 \\
\hline LD trucks & -489 & 743 & -0.02 & -0.15 & 0.00 & 0.02 & -0.01 & -0.01 & 0.00 & 0.02 & 0.02 \\
\hline MD trucks & $-254,340$ & 386,611 & -1.71 & -20.94 & 3.96 & 11.94 & -0.83 & -6.62 & -0.15 & 8.10 & 12.07 \\
\hline HD trucks & $-911,966$ & $1,386,239$ & -17.75 & -138.47 & -3.32 & 42.81 & -4.20 & -23.46 & -0.52 & 29.04 & 43.26 \\
\hline Buses & $-22,351$ & 33,975 & -0.82 & -0.38 & -0.61 & 1.05 & -0.03 & -0.59 & -0.01 & 0.71 & 1.06 \\
\hline Subtotal & $-1,191,250$ & $1,810,768$ & -20.39 & -160.73 & 0.01 & 55.93 & -5.12 & -30.73 & -0.68 & 37.93 & 56.51 \\
\hline \multicolumn{12}{|l|}{ Off-Road Mobile Sources } \\
\hline Trains (locomotives) & $-117,668$ & 178,862 & -3.14 & -7.22 & -8.27 & 5.52 & -0.86 & -22.45 & -0.07 & 3.75 & 5.58 \\
\hline Vessels & $-102,695$ & 156,103 & -3.54 & -1.51 & 0.22 & 4.82 & .0 .97 & -43.08 & -0.06 & 3.27 & 4.87 \\
\hline $\begin{array}{l}\text { Farm equipment } \\
\text { (diesel) }\end{array}$ & $-84,319$ & 128,169 & -4.61 & -15.20 & -7.27 & 3.96 & -2.12 & -15.77 & -0.05 & 2.69 & 4.00 \\
\hline $\begin{array}{l}\text { LD non-farm equipment } \\
\text { (diesel) }\end{array}$ & $-19,357$ & 29,424 & -1.05 & -3.48 & -1.54 & 0.91 & -0.47 & -3.62 & -0.01 & 0.62 & 0.92 \\
\hline $\begin{array}{l}\text { HD non-farm equipment } \\
\text { (diesel) }\end{array}$ & $-133,277$ & 202,588 & -4.17 & -9.40 & -6.52 & 6.26 & -1.66 & -25.28 & -0.08 & 4.24 & 6.32 \\
\hline $\begin{array}{l}\text { Refrigeration units } \\
\text { (diesel) }\end{array}$ & $-10,013$ & 15,221 & -0.67 & -2.49 & -0.82 & 0.47 & -0.29 & -1.86 & -0.01 & 0.32 & 0.47 \\
\hline Subtotal & $-467,329$ & 710,367 & -17.17 & -39.30 & -24.20 & 21.94 & -6.37 & -112.07 & -0.27 & 14.88 & 22.17 \\
\hline Stationary Sources & $-21,712$ & 33,003 & -0.94 & -5.80 & -2.69 & 1.02 & -1.15 & -3.92 & -0.01 & 0.69 & 1.03 \\
\hline Total - All Sources & $-1,680,291$ & $2,554,138$ & -38.50 & -205.83 & -26.88 & 78.88 & -12.64 & -146.72 & -0.96 & 53.51 & 79.71 \\
\hline
\end{tabular}


Table 33 Net Changes in Energy and Emissions Caused by Production and Combustion of Control Scenario 4 Fuel (DME),

\begin{tabular}{|c|c|c|c|c|c|c|c|c|c|c|c|}
\hline \multirow[b]{2}{*}{ Source Category } & \multicolumn{2}{|c|}{$\begin{array}{c}\text { Changes in Daily Fuel } \\
\text { Energy }\left(10^{6} \mathrm{Btu}\right)\end{array}$} & \multicolumn{8}{|c|}{ Changes in Emissions (ton/day) } & \multirow{2}{*}{$\begin{array}{c}\text { GHGs } \\
\left(\mathrm{CO}_{2} \text {-equivalent }\right) \\
\left(10^{3}\right)\end{array}$} \\
\hline & Diesel & FT & ROG & co & $\mathrm{NO}_{\mathrm{x}}$ & $\mathrm{CO}_{2}\left(10^{3}\right)$ & $\mathbf{P M}_{10}$ & so $_{\mathrm{x}}$ & $\mathrm{N}_{2} \mathrm{O}$ & $\mathrm{CH}_{4}$ & \\
\hline \multicolumn{12}{|l|}{ On-Road Mobile Sources } \\
\hline Autos & $-2,105$ & 2,757 & -0.08 & 1.21 & -0.35 & 0.03 & -0.08 & -0.05 & 0.00 & 0.08 & 0.03 \\
\hline LD trucks & .489 & 640 & -0.02 & 0.22 & -0.07 & 0.01 & -0.02 & -0.01 & 0.00 & 0.02 & 0.01 \\
\hline MD trucks & $-254,340$ & 333,086 & -1.00 & 39.86 & -14.24 & 3.97 & -1.55 & -6.74 & -0.15 & 8.85 & 4.11 \\
\hline HD trucks & $-911,966$ & $1,194,321$ & -13.83 & 231.82 & -153.12 & 14.24 & -7.69 & -23.92 & -0.55 & 31.03 & 14.72 \\
\hline Buses & $-22,351$ & 29,271 & -0.68 & 1.46 & -6.85 & 0.35 & -0.06 & -0.60 & -0.01 & 0.87 & 0.36 \\
\hline $\begin{array}{l}\text { Subtotal } \\
\text { Off-Road Mobile Sources }\end{array}$ & $-1,191,250$ & $1,560,075$ & -15.61 & 274.57 & -174.63 & 18.60 & -9.40 & -31.32 & -0.72 & 40.86 & 19.24 \\
\hline Trains (locomotives) & $-117,668$ & 154,099 & -2.54 & 14.98 & -65.44 & 1.84 & -1.56 & -22.51 & -0.07 & 4.02 & 1.90 \\
\hline Vessels & $-102,695$ & 134,491 & -2.92 & 6.35 & -13.80 & 1.60 & -1.74 & -43.13 & -0.06 & 3.51 & 1.66 \\
\hline $\begin{array}{l}\text { Farm equipment } \\
\text { (diesel) }\end{array}$ & $-84,319$ & 110,425 & -3.90 & 24.80 & -54.74 & 1.32 & -3.76 & -15.81 & -0.05 & 2.89 & 1.36 \\
\hline $\begin{array}{l}\text { LD non-farm equipment } \\
\text { (diesel) }\end{array}$ & $-19,357$ & 25,351 & -0.88 & 5.67 & -11.80 & 0.30 & -0.83 & -3.63 & -0.01 & 0.68 & 0.31 \\
\hline $\begin{array}{l}\text { HD non-farm equipment } \\
\text { (diesel) }\end{array}$ & $-133,277$ & 174,541 & -3.41 & 18.68 & -57.57 & 2.08 & -2.97 & -25.35 & -0.08 & 4.57 & 2.15 \\
\hline $\begin{array}{l}\text { Refrigeration units } \\
\text { (diesel) }\end{array}$ & $-10,013$ & 13,114 & -0.57 & 3.91 & -6.27 & 0.16 & -0.51 & -1.87 & -0.01 & 0.39 & 0.16 \\
\hline Subtotal & $-467,329$ & 612,020 & -14.22 & 74.39 & -209.63 & 7.30 & -11.37 & -112.31 & -0.28 & 16.05 & 7.55 \\
\hline Stationary Sources & $-21,712$ & 28,434 & -0.79 & 9.02 & -18.87 & 0.34 & -2.04 & -3.93 & -0.01 & 0.67 & 0.35 \\
\hline Total - All Sources & $-1,680,291$ & $2,200,529$ & -30.61 & 357.98 & -403.13 & 26.24 & -22.82 & -147.56 & -1.02 & 57.58 & 27.13 \\
\hline
\end{tabular}


Table 34 Summary of Energy Increases for the Various Scenarios for 2010 (in $10^{6} \mathrm{Btu} / \mathrm{d}$ )

\begin{tabular}{|c|c|c|c|c|c|c|c|c|c|c|}
\hline \multirow[b]{2}{*}{ Source Category } & \multirow[b]{2}{*}{$\begin{array}{c}\text { Base Case } \\
\text { Combustion } \\
\text { Only }\end{array}$} & \multirow[b]{2}{*}{$\begin{array}{c}\text { Base Case } \\
\text { Combustion } \\
\text { and } \\
\text { Production } \\
\end{array}$} & \multicolumn{2}{|c|}{ Control Scenario 1} & \multicolumn{2}{|c|}{ Control Scenario 2} & \multicolumn{2}{|c|}{ Control Scenario 3} & \multicolumn{2}{|c|}{ Control Scenario 4} \\
\hline & & & $\begin{array}{c}\text { Combustion } \\
\text { Only }\end{array}$ & $\begin{array}{c}\text { Combustion } \\
\text { and } \\
\text { Production }\end{array}$ & $\begin{array}{c}\text { Combustion } \\
\text { Only }\end{array}$ & $\begin{array}{c}\text { Combustion } \\
\text { and } \\
\text { Production }\end{array}$ & $\begin{array}{c}\text { Combustion } \\
\text { Only }\end{array}$ & $\begin{array}{c}\begin{array}{c}\text { Combustion } \\
\text { and } \\
\text { Production }\end{array} \\
\end{array}$ & $\begin{array}{c}\text { Combustion } \\
\text { Only }\end{array}$ & $\begin{array}{c}\text { Combustion } \\
\text { and } \\
\text { Production }\end{array}$ \\
\hline \multicolumn{11}{|l|}{ On-Road Mobile Sources } \\
\hline Autos & 1,822 & 2,105 & 202 & 2,548 & 202 & 2,548 & 0 & 3,200 & 0 & 2,757 \\
\hline LD trucks & 423 & 489 & 47 & 592 & 47 & 592 & 0 & 743 & 0 & 640 \\
\hline MD trucks & 220,206 & 254,340 & 83,201 & 381,835 & 34,452 & 252,355 & 0 & 386,611 & 0 & 333,086 \\
\hline HD trucks & 789,575 & 911,966 & 637,436 & $1,795,880$ & 181,180 & 584,046 & 0 & $1,386,239$ & 0 & $1,194,321$ \\
\hline Buses & 19,351 & 22,351 & 13,083 & 40,818 & 10,546 & 9,900 & 0 & 33,975 & 0 & 29,271 \\
\hline Subtotal & $1,031,377$ & $1,191,250$ & 733,970 & $2,221,673$ & 226,428 & 849,441 & 0 & $1,810,768$ & 0 & $1,560,075$ \\
\hline \multicolumn{11}{|l|}{ Off-Road Mobile Sources } \\
\hline Trains (locomotives) & 101,876 & 117,668 & 18,338 & 158,552 & 5,043 & 127,345 & 0 & 178,862 & 0 & 154,099 \\
\hline Vessels & 88,913 & 102,695 & 16,004 & 138,377 & 4,401 & 111,141 & 0 & 156,103 & 0 & 134,491 \\
\hline Farm equipment (diesel) & 73,003 & 84,319 & 18,251 & 114,842 & 18,251 & 114,842 & 0 & 128,169 & 0 & 110,425 \\
\hline $\begin{array}{l}\text { LD non-farm equipment } \\
\text { (diesel) }\end{array}$ & 16,760 & 19,357 & 1,676 & 23,201 & 1,676 & 23,201 & 0 & 29,424 & 0 & 25,351 \\
\hline $\begin{array}{l}\text { HD non-farm equipment } \\
\text { (diesel) }\end{array}$ & 115,390 & 133,277 & 28,848 & 181,522 & 28,848 & 181,522 & 0 & 202,588 & 0 & 174,541 \\
\hline Refrigeration units (diesel) & 8,670 & 10,013 & 867 & 12,002 & 867 & 12,002 & 0 & 15,221 & 0 & 13,114 \\
\hline Subtotal & 404,611 & 467,329 & 83,983 & 628,495 & 59,085 & 570,052 & 0 & 710,367 & 0 & 612,020 \\
\hline Stationary Sources & 18,798 & 21,712 & 3,384 & 29,255 & 930 & 23,497 & 0 & 33,003 & 0 & 28,434 \\
\hline Total - All Sources & $1,454,786$ & $1,680,291$ & 821,336 & $2,879,423$ & 286,444 & $1,442,990$ & 0 & $2,554,138$ & 0 & $2,200,529$ \\
\hline
\end{tabular}


Table 35 Summary of $\mathrm{NO}_{x}$ Emission Changes for the Various Scenarios for 2010 (in ton/d)

\begin{tabular}{|c|c|c|c|c|c|c|c|c|c|c|}
\hline \multirow[b]{2}{*}{ Source Category } & \multirow[b]{2}{*}{$\begin{array}{c}\begin{array}{c}\text { Base Case } \\
\text { Combustion } \\
\text { Only }\end{array} \\
\end{array}$} & \multirow[b]{2}{*}{$\begin{array}{c}\text { Base Case } \\
\text { Combustion } \\
\text { and } \\
\text { Production }\end{array}$} & \multicolumn{2}{|c|}{ Control Scenario 1} & \multicolumn{2}{|c|}{ Control Scenario 2} & \multicolumn{2}{|c|}{ Control Scenario 3} & \multicolumn{2}{|c|}{ Control Scenario 4} \\
\hline & & & $\begin{array}{c}\text { Combustion } \\
\text { Only }\end{array}$ & $\begin{array}{c}\text { Combustion } \\
\text { and } \\
\text { Production }\end{array}$ & $\begin{array}{c}\text { Combustion } \\
\text { Only }\end{array}$ & $\begin{array}{c}\text { Combustion } \\
\text { and } \\
\text { Production }\end{array}$ & $\begin{array}{c}\text { Combustion } \\
\text { Only }\end{array}$ & $\begin{array}{c}\text { Combustion } \\
\text { and } \\
\text { Production }\end{array}$ & $\begin{array}{c}\text { Combustion } \\
\text { Only }\end{array}$ & $\begin{array}{c}\text { Combustion } \\
\text { and } \\
\text { Production }\end{array}$ \\
\hline \multicolumn{11}{|l|}{ On-Road Mobile Sources } \\
\hline Autos & 1 & 1 & -1 & -1 & .1 & -1 & 0 & 0 & 0 & 0 \\
\hline LD trucks & 0 & 0 & 0 & 0 & 0 & 0 & 0 & 0 & 0 & 0 \\
\hline MD trucks & 39 & 45 & 7 & 12 & -5 & 1 & -3 & 4 & -19 & -14 \\
\hline HD trucks & 351 & 371 & 8 & 41 & .44 & -10 & .29 & -3 & -169 & -153 \\
\hline Buses & 15 & 16 & 6 & 7 & -5 & -4 & -1 & -1 & -7 & -7 \\
\hline \multirow{2}{*}{\multicolumn{11}{|c|}{ Off-Road Mobile Sources }} \\
\hline & & & & & & & & & & \\
\hline Trains (locomotives) & 140 & 143 & -132 & -120 & -54 & -44 & -12 & -8 & -68 & -65 \\
\hline Vessels & 32 & 35 & -30 & -20 & -12 & -4 & -3 & 0 & -16 & .14 \\
\hline Farm equipment (diesel) & 117 & 118 & -94 & -92 & .94 & -92 & -10 & -7 & .56 & .55 \\
\hline $\begin{array}{l}\text { LD non-karm equipment } \\
\text { (diesel) }\end{array}$ & 25 & 26 & -19 & -19 & -19 & -19 & -2 & -2 & -12 & -12 \\
\hline $\begin{array}{l}\text { HD non-farm equipment } \\
\text { (diesel) }\end{array}$ & 124 & 127 & -118 & -116 & -118 & -116 & -10 & -7 & -60 & -58 \\
\hline Refrigeration units (diesel) & 13 & 14 & -10 & -10 & -10 & -10 & -1 & -1 & -6 & -6 \\
\hline Subtotal & 452 & 462 & -403 & -377 & -307 & -285 & -37 & -24 & -218 & -210 \\
\hline $\begin{array}{l}\text { Stationary Sources } \\
\text { Total All Sources }\end{array}$ & $\begin{array}{r}40 \\
897-10\end{array}$ & $\begin{array}{r}40 \\
035\end{array}$ & $\begin{array}{r}-34 \\
-417\end{array}$ & $\begin{array}{r}-32 \\
-350\end{array}$ & $\begin{array}{r}13 \\
-348\end{array}$ & $\begin{array}{r}15 \\
-284\end{array}$ & -3 & $\begin{array}{r}-3 \\
-27\end{array}$ & $\begin{array}{r}-19 \\
-433\end{array}$ & $\begin{array}{r}-19 \\
-003\end{array}$ \\
\hline Total - All Sources & & 935 & -417 & -350 & -348 & -284 & -74 & & -433 & -403 \\
\hline
\end{tabular}


Changes in $\mathrm{PM}_{10}$ emissions are summarized in Table 36. All of the control scenarios result in $\mathrm{PM}_{10}$ reductions, but the greatest reductions come from Scenario 2 and the least from Scenario 3.

Table 37 shows that all of the control scenarios result in GHG emission increases. The lowest increases result from Scenarios 2 and 4.

No single scenario yields the lowest combined impact for all of the important components of emissions and energy use. While Scenario 1 would likely be the most straightforward to implement, it would also consume the greatest amount of energy. Use of FTD would also be quite easy to implement, but would also consume a large amount of energy. Scenario 2, which employs a substantial amount of $\mathrm{NG}$, is perhaps the best choice to minimize overall negative effects on energy use and the environment. 
Table 36 Summary of $\mathrm{PM}_{10}$ Emission Changes for the Various Scenarios for 2010 (in ton/d)

\begin{tabular}{|c|c|c|c|c|c|c|c|c|c|c|}
\hline \multirow[b]{2}{*}{ Source Category } & \multirow[b]{2}{*}{$\begin{array}{c}\text { Base Case } \\
\text { Combustion } \\
\text { Only } \\
\end{array}$} & \multirow[b]{2}{*}{$\begin{array}{c}\text { Base Case } \\
\text { Combustion } \\
\text { and } \\
\text { Production }\end{array}$} & \multicolumn{2}{|c|}{ Control Scenario 1} & \multicolumn{2}{|c|}{ Control Scenario 2} & \multicolumn{2}{|c|}{ Control Scenario 3} & \multicolumn{2}{|c|}{ Control Scenario 4} \\
\hline & & & $\begin{array}{c}\text { Combustion } \\
\text { Only }\end{array}$ & $\begin{array}{c}\text { Combustion } \\
\text { and } \\
\text { Production }\end{array}$ & $\begin{array}{c}\text { Combustion } \\
\text { Only }\end{array}$ & $\begin{array}{c}\text { Combustion } \\
\text { and } \\
\text { Production }\end{array}$ & $\begin{array}{c}\text { Combustion } \\
\text { Only }\end{array}$ & $\begin{array}{c}\text { Combustion } \\
\text { and } \\
\text { Production }\end{array}$ & $\begin{array}{c}\text { Combustion } \\
\text { Only }\end{array}$ & $\begin{array}{c}\text { Combustion } \\
\text { and } \\
\text { Production }\end{array}$ \\
\hline \multicolumn{11}{|l|}{ On-Road Mobile Sources } \\
\hline Autos & 0 & 0 & 0 & 0 & 0 & 0 & 0 & 0 & 0 & 0 \\
\hline LD trucks & 0 & 0 & 0 & 0 & 0 & 0 & 0 & 0 & 0 & 0 \\
\hline MD trucks & 3 & 3 & -2 & -1 & -2 & -2 & -1 & -1 & -1 & -2 \\
\hline HD trucks & 15 & 17 & -8 & -5 & -13 & -11 & -4 & -4 & -7 & -8 \\
\hline Buses & 0 & 0 & 0 & 0 & 0 & 0 & 0 & 0 & 0 & 0 \\
\hline \multirow{2}{*}{\multicolumn{10}{|c|}{ Off-Road Mobile Sources }} & -9 \\
\hline & & & & & & & & & & \\
\hline Trains (locomotives) & 3 & 3 & -1 & 0 & 0 & 1 & -1 & -1 & -2 & -2 \\
\hline Vessels & 3 & 4 & -1 & 0 & 0 & 1 & -1 & -1 & -2 & -2 \\
\hline Farm equipment (diesel) & 7 & 8 & -7 & -7 & -7 & .7 & -2 & -2 & -4 & -4 \\
\hline $\begin{array}{l}\text { LD non-farm equipment } \\
\text { (diesel) }\end{array}$ & 2 & 2 & -2 & -2 & -2 & -2 & 0 & 0 & -1 & -1 \\
\hline $\begin{array}{l}\text { HD non-farm equipment } \\
\text { (diesel) }\end{array}$ & 6 & 6 & -6 & -5 & -6 & -5 & -2 & -2 & -3 & -3 \\
\hline Refrigeration units (diesel) & 1 & 1 & -1 & -1 & -1 & -1 & 0 & 0 & -1 & -1 \\
\hline Subtotal & 22 & 23 & -17 & -14 & -16 & -13 & -6 & -6 & -11 & \\
\hline Stationary Sources & $\begin{array}{c}4 \\
44\end{array}$ & $\begin{array}{r}4 \\
48\end{array}$ & -1 & -1 & 3 & 3 & -1 & -1 & -2 & -2 \\
\hline Total - All Sources & & & & & & & & & & \\
\hline
\end{tabular}


Table 37 Summary of GHG Emission Changes for the Various Scenarios for 2010 (in ton/d)

\begin{tabular}{|c|c|c|c|c|c|c|c|c|c|c|}
\hline \multirow[b]{2}{*}{ Source Category } & \multirow[b]{2}{*}{$\begin{array}{c}\text { Base Case } \\
\text { Combustion } \\
\text { Only }\end{array}$} & \multirow{2}{*}{$\begin{array}{c}\text { Base Case } \\
\text { Combustion } \\
\text { and } \\
\text { Production }\end{array}$} & \multicolumn{2}{|c|}{ Control Scenario 1} & \multicolumn{2}{|c|}{ Control Scenario 2} & \multicolumn{2}{|c|}{ Control Scenario 3} & \multicolumn{2}{|c|}{ Control Scenario 4} \\
\hline & & & $\begin{array}{c}\text { Combustion } \\
\text { Only }\end{array}$ & $\begin{array}{c}\text { Combustion } \\
\text { and } \\
\text { Production }\end{array}$ & $\begin{array}{c}\text { Combustion } \\
\text { Only }\end{array}$ & $\begin{array}{c}\text { Combustion } \\
\text { and } \\
\text { Production }\end{array}$ & $\begin{array}{c}\text { Combustion } \\
\text { Only }\end{array}$ & $\begin{array}{c}\text { Combustion } \\
\text { and } \\
\text { Production }\end{array}$ & $\begin{array}{c}\text { Combustion } \\
\text { Only }\end{array}$ & $\begin{array}{c}\text { Combustion } \\
\text { and } \\
\text { Production }\end{array}$ \\
\hline \multicolumn{11}{|l|}{ On-Road Mobile Sources } \\
\hline Autos & 0 & 0 & 0 & 0 & 0 & 0 & 0 & 0 & 0 & 0 \\
\hline LD trucks & 0 & 0 & 0 & 0 & 0 & 0 & 0 & 0 & 0 & 0 \\
\hline MD trucks & 19 & 23 & 8 & 12 & -1 & 2 & 0 & 12 & -1 & 4 \\
\hline HD trucks & 69 & 81 & 69 & 91 & -1 & 12 & 2 & 43 & -3 & 15 \\
\hline Buses & 2 & 2 & 3 & 4 & -1 & 0 & 0 & 1 & 0 & 0 \\
\hline Subtotal & 91 & 106 & 80 & 106 & -3 & 14 & 2 & 57 & -4 & 19 \\
\hline \multicolumn{11}{|l|}{ Oft-Road Mobile Sources } \\
\hline Trains (locomotives) & 9 & 10 & -1 & 1 & 0 & 2 & 0 & 6 & 0 & 2 \\
\hline Vessels & 8 & 9 & -1 & 1 & 0 & 1 & 0 & 5 & 0 & 2 \\
\hline Farm equipment (diesel) & 6 & 7 & 2 & 3 & 2 & 3 & 0 & 4 & 0 & 1 \\
\hline $\begin{array}{l}\text { LD non-farm equipment } \\
\text { (diesel) }\end{array}$ & 1 & 2 & 0 & 0 & 0 & -2 & 0 & 1 & 0 & 0 \\
\hline $\begin{array}{l}\text { HD non-farm equipment } \\
\text { (diesel) }\end{array}$ & 10 & 12 & 2 & 4 & 2 & 5 & 0 & 6 & 0 & 2 \\
\hline Refrigeration units (diesel) & 1 & 1 & 0 & 0 & 0 & -6 & 0 & 0 & 0 & 0 \\
\hline Subtotal & 36 & 42 & 1 & 7 & 3 & 4 & 1 & 22 & -2 & 8 \\
\hline Stationary Sources & 2 & 2 & 0 & 0 & 1 & 2 & 0 & 1 & 0 & 0 \\
\hline Total - All Sources & 128 & 150 & 81 & 114 & 2 & 19 & 3 & 80 & -6 & 27 \\
\hline
\end{tabular}




\section{Section 4 \\ Conclusions and Discussion}

Experts anticipate that California will move forward to expedite substitution of (conventional) diesel fuel with formulations that can meet the challenges posed by CARB's August 1998 decision on diesel particulate toxicity - that is, fuel formulations characterized by lower particulate mass in the exhaust. Examples include stringently reduced-sulfur diesel, liquefied and compressed NG, and FTD. Biodiesel, as mentioned above, is not a near-term option because of supply and source constraints and the fact that, while it lowers particulates, biodiesel (according to most tests) increases $\mathrm{NO}_{\mathrm{x}}$ emissions from current engines. Whatever option is chosen will be accompanied by economic and safety effects. Some of the more important effects are discussed below.

If California was forced into a "best-of-worst-case" situation (see Section 2), essentially that described under Scenario 1, the population of MD and HD trucks, in particular, would have to increase by as much as $50 \%$ to accommodate projected requirements for road freight tonnage. Because all of these trucks would be fueled by gasoline, the incremental daily demand for gasoline by trucks in California (with diesel demand zeroed out) would be about 15 million gallons, or 350,000 barrels. This amount is just under half of California's current daily gasoline consumption - about $37 \%$ of projected year-2010 daily gasoline demand (without diesel prohibition) - and does not include the additional 7 million gallons required by other vehicle types and in "upstream" fuel production and transport activities. An overall daily diversion of this magnitude (over one-half million barrels) from normal refinery production uses could impose significant short-term stress on domestic refiners and lead to spot shortages elsewhere in the United States. California motorists would also likely have to pay a substantial premium for the additional gasoline.

Not surprisingly, we also observe a large increase in mass emissions of CO and GHGs under this scenario. Daily demand for LNG as a transportation and process fuel would increase from the current negligible level to 4.5 million gallons (60,000 diesel-equivalent barrels) in 2010 . (This increase in demand is affected not just by the decrease in fuel economy when switching to CNG or LNG but also by the approximately $15 \%$ added weight of CNG-fueled buses, relative to their diesel counterparts, because of the weight of the fuel cylinders. A large transit bus fueled by CNG or LNG would weigh approximately 15 tons, rather than the 13.5 tons that today's dieselfueled buses weigh.) Transport and distribution (by traditional means) of this quantity of NG in a cryogenically stored form would increase the risk of catastrophic accidents and explosions in the ports of San Francisco, Oakland, and Long Beach, as well as during transit by highway tankers. Although we have no conclusive evidence at this point that more trucks on the road to deliver the replacement fuel would result in more (and more serious) highway accidents than in an "alldiesel" environment, this possibility cannot be ruled out.

The intermediate case, Scenario 2, has mixed effects. With diesel pilot fuel used for NGfueled HD vehicles, gasoline demand would increase by only 8.5 million gallons per day overall (about $21 \%$ over baseline). This amount is just over a third of the Scenario 1 increase, and 
California may be able to meet this demand without severe disruption in 2010 if we assume a modest increase in worldwide capacity by that time. By contrast, NG demand would increase by 3.6 million diesel-equivalent gallons per day (about 7\% over baseline for all uses in the state); of this total, the CNG demand would represent an incremental 192 million standard cubic feet. It is not likely that this increase in daily flow could be supplied $100 \%$ by domestic pipelines, so NG importation would probably be necessary, initially from Canada and Mexico but then from abroad. The safety hazards identified for Scenario 1 would not be eliminated for this scenario. Increases in emissions would be lower for this scenario than for Scenario 1. Added CO and GWP-weighted GHG emissions would be lower, although methane emissions would increase because of greater $\mathrm{NG}$ consumption. $\mathrm{SO}_{\mathrm{x}}$ and $\mathrm{PM}_{10}$ emissions would be reduced by a greater amount than in Scenario 1, but $\mathrm{NO}_{\mathrm{x}}$ reduction would be less dramatic.

Each "best case" alternative (Scenarios 3 and 4) has unique characteristics. The FTD case (Scenario 3) would result in an almost 20-million-gallon-per-day demand for that synthetic fuel, including its use as a process fuel. This represents an increase of 7 million diesel-equivalent gallons over the quantity of diesel displaced. There is absolutely no indication that inherently safe production capacity to meet that demand can be on line by 2010. If we assume it can, current indications are that emissions of priority pollutants would decline, while GHG emissions could rise by an amount intermediate to the increases caused by Scenarios 1 and 2. Reduction in $\mathrm{SO}_{\mathrm{x}}$ emissions would be especially dramatic; fine particulate reductions would be less so.

The DME case (Scenario 4) would require somewhat less diesel-equivalent energy for replacement fuel (17 million gallons) and would result in a lower GHG increase and greater $\mathrm{PM}_{10}$ and $\mathrm{NO}_{\mathrm{x}}$ decreases than would use of FTD, but it would actually increase $\mathrm{CO}$ emissions relative to the base case. Again, the existence of DME production capacity to meet such a demand by 2010 is highly speculative; current and projected petroleum prices appear unlikely to create incentives to drive a rapid expansion in capacity.

It is important to note that this study is not a cost/benefit analysis. Dollar value trade-offs for damages avoided by reducing particulate, $\mathrm{NO}_{\mathrm{x}}$, and $\mathrm{SO}_{\mathrm{x}}$ emissions (net benefits) have not been compared to general increases in energy use, GHGs, and other criteria pollutants. Similarly, the net benefits revealed by such comparisons, if any, would have to be shown to exceed the costs of making the changes in fuels and vehicles for each scenario. Such analyses were beyond the scope of this study. 


\section{Section 5 References}

American Public Transit Association, 1996, 1996 Transit Fact Book, Jan.

APTA - see American Public Transit Association.

Bekken, M., 1994, California Air Resources Board, personal communication with D.M. Rote, Argonne National Laboratory, regarding June 1978 particulate matter emissions data, Sept. 16.

California Air Resources Board, 1995, Emission Inventory Procedural Manual, Vol. 3: Methods For Assessing Area Source Emissions, Sept.

California Air Resources Board, 1997a, "Emissions by Category, 1995 Estimated Emissions, Statewide," and "Emissions by Category, 2010 Forecasted Emissions," available at http://www.arb.ca.gov/ ceidars/emssumcat.query?F_DIV=O\&F_YR=1995\&F_AREA=CA.

California Air Resources Board, 1997b, "Predicted California Vehicle Emissions, Ozone Planning Inventory, MVEI7G Emission Factors Scenario," computer model run date May 27, available at http://www.arb.ca. gov/msei/pubs/sw_scab.pdf.

California Air Resources Board, 1998a, Resolution 98-35, State of California Air Resources Board, Aug. 27.

California Air Resources Board, 1998b, Proposed Identification of Diesel Exhaust as a Toxic Air Contaminant: Part A, Exposure Assessment, Stationary Source Division, Sacramento, California, Feb.

CARB - see California Air Resources Board.

Davila, P., 1998, Eurodyn, Seneca Group, Arlington, Texas, letter to D.M. Rote, Argonne National Laboratory, June 1 (transmittal included 3,572-hp unit Eurodyne Gas Turbine description and data sheets, ref. no. ECC-101-1).

Delucchi, M.A., 1997, Emissions of Non-CO $\mathrm{C}_{2}$ Greenhouse Gases from the Production and Use of Transportation Fuels and Electricity, report no. UCD-ITS-RR-97-5, Institute of Transportation Studies, University of California at Davis, Davis, Calif.

DOE and EIA - see U.S. Department of Energy and Energy Information Administration.

EF \& EE - see Engine, Fuel, and Emissions Engineering, Inc.

Engine, Fuel, and Emissions Engineering, Inc., 1993, Controlling Locomotive Emissions in California, prepared for the California Air Resources Board, Oct. 
EPA - see U.S. Environmental Protection Agency.

Federal Transit Administration, 1997, New Bus Model Testing Program, Federal Transit Administration, Dec. 12, available at http://www.pti.psu.edu/open/fta/ftaopen.htm

Fleisch, T.H., 1995, “A New Clean Diesel Technology: Demonstration of ULEV Emissions on a Navistar Diesel Engine Fueled with Dimethyl Ether," SAE paper 950061, Society of Automotive Engineers, Warrendale, Penn.

Fleisch, T.H., and P.C. Meurer, 1995, "DME, The Diesel Fuel for the 21st Century?," presented at the AVL Conference Engine and Environment 1995, Graz, Austria, Aug.

Garshick, E., et al., 1998, "A Retrospective Cohort Study of Lung Cancer and Diesel Exhaust Exposure in Railroad Workers," Am. Rev. Respir. Dis. 137:820-825.

FTA - see Federal Transit Administration.

Hansen, J.B., and S.E. Mikkelsen, 1998, "DME as Neat Diesel Fuel," presented at the Conference on Gas-to-Liquids Processing Technology, San Antonio, Texas, March 18-20.

Health Effects Institute, 1999, Diesel Emissions and Lung Cancer: Epidemiology and Quantitative Risk Assessments, a special report of the Institute's Diesel Epidemiology Expert Panel, North Andover, Mass., June.

HEI - see Health Effects Institute.

Inside EPA's Mobile Source Report, 1998a, Inside Washington Publishers, Washington, D.C., May 7, pp. 7, 14.

Inside EPA's Mobile Source Report, 1998b, Inside Washington Publishers, Washington, D.C., March 26, pp. 8-9.

Jensen, P., 1998, Energy Conversions Inc., letter to D.M. Rote, Argonne National Laboratory, March 12 (transmittal provided included data for emission tests performed at Southwest Research Institute, brochure entitled "Natural Gas Alternatives: A Retrofit System to Convert a Locomotive to Natural Gas Operation" by S.P. Jensen, ICE-Vol.21, Natural Gas and Alternative Fuels for Engines, ASME 1994, "Effects of Fuel Gas Mixtures on Power Limits in a Dual-Fuel Engine" by M.J. Gillispie and M.A. Jensen, ICE-Vol. 21, and other references to the Burlington Northern Railroad field tests of a converted dual-fuel engine.)

Leet, J.A., 1998, "Potential Application of Fischer-Tropsch Fuels," presented at the Conference on Gas-to-Liquids Processing Technology, San Antonio, Texas, March 18-20.

McGregor, D., 1994, Engine, Fuel, and Emissions Engineering, Inc., personal communication with D.M. Rote, Argonne National Laboratory, regarding March 16, 1982, idle emissions and horsepower v. notch setting data, Sept. 16. 
MSR - See Inside EPA's Mobile Source Report.

Mull, K.E., 1995, Allied Signal Aerospace, letter to D.M. Rote, Argonne National Laboratory, Feb. 24 (transmittal included data sheets on the TF40 engine burning \#2 diesel fuel).

National Renewable Energy Laboratory, 1996a, Alternative-Fueled Truck Demonstration Natural Gas Program: Caterpillar G3406LE Development and Demonstration, Fifth Annual Report to Congress, National Renewable Energy Laboratory, Golden Colo., available from National Technical Information Service or at http://www.afdc.nrel.gov/demoproj/hdv/hdvrpts /vonsec1.html.

National Renewable Energy Laboratory, 1996b, "Alternative Fuel Transit Buses, Final Results from the NREL Vehicle Evaluation Program," Oct., available at http://www.afdc.nrel.gov.

NREL - see National Renewable Energy Laboratory.

Olsen, L.E., 1997, private consultant (formerly with Burlington Northern Railroad), letter to L. Torres, Empresas Polar, May 20.

Olsen, L.E., 1998, “The Natural Gas Locomotive at Burlington Northern Railroad," Pacific Rim TransTech Conference, Seattle, Wash., July.

Railroad Facts, 1997, Association of American Railroads, Information and Public Affairs Department.

Rheinhart, M., 1998, Caterpillar, Inc. Engine Center, Lafayette, Indiana, personal communication to D.M. Rote, Argonne National Laboratory, June 2.

Steenland, K., et al., 1998, "Diesel Exhaust and Lung Cancer in the Trucking Industry: ExposureResponse Analysis and Risk Assessment," Am. J. Ind. Med. 34:220-228.

TIUS - see Truck Inventory and Use Survey.

Truck Inventory and Use Survey, 1992, "California Truck Registration and Usage, Summary of California Based Trucks by GVW Class, Fuel Type, and Engine Displacement, 1992,” microdata CD issued in June.

U.S. Department of Energy and Energy Information Administration, 1996, "Fuel Oil and Kerosene Sales" available at http://www.eia.doe.gov/oil_gas/petroleum/pet frame.html.

U.S. Environmental Protection Agency, 1998, Draft 1998 Inventory for U.S. Greenhouse Gas Emissions and Sinks (1990-1996), Washington, D.C., March 19.

Wang, M. Q., 1996, GREET 1.0 - Transportation Fuel-Cycle Model: Methodology and Use, ANL/ESD-33, Argonne National Laboratory, Argonne, Ill., June. 